University of Redlands

\title{
Managing Marine Mammal Observations Using a Volunteered Geographic Information Approach
}

A Major Individual Project submitted in partial satisfaction of the requirements

for the degree of Master of Science in Geographic Information Systems

by

Melodi C. King

Douglas M. Flewelling, Ph.D., Committee Chair

Lei Lani Stelle, Ph.D.

December 2012 
Managing Marine Mammal Observations Using a Volunteered Geographic Information Approach

Copyright (C) 2012

by

Melodi C. King 

The report of Melodi King is approved.

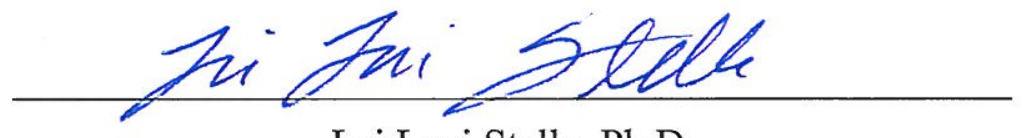

Lei Lani Stelle, Ph.D.

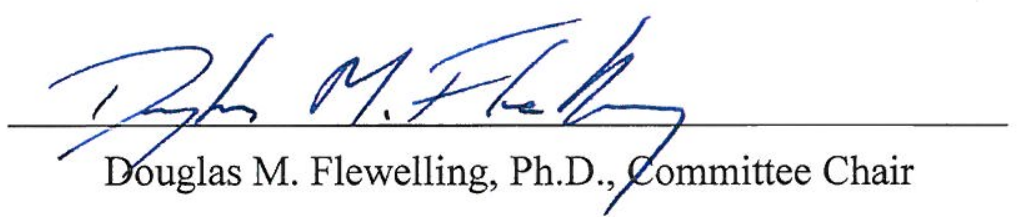

December 2012 


\section{Acknowledgements}

First and foremost, I would like to express my sincere gratitude and thanks to my advisor and committee chair, Doug Flewelling. Doug consistently gave me creative freedom during development and acted as a sounding board and mentor without being overbearing with the project.

I would also like to thank my client, Dr. Lei Lani Stelle. I could not have asked for a better client relationship for my major individual project. Lei Lani was enthusiastic about the project and always made herself available when I had questions. Dr. Stelle and her husband, Shane Keena, always made me feel very welcome and comfortable when we were out on the boat. A special thanks to Shane for the wonderful photos donated to the project and the adventurous whale watching trip.

Of course, the whale watching trips would not have possible without the help from Captain Larry of Davies Locker Whale Watch. Captain Larry always made his boat available for me if I needed to test the mobile application.

Additional thanks to the entire MS GIS department faculty and staff. A big hug and thanks to Debbie Riley for always making sure that I had a cup of green tea. Doug Flewelling, Mark Kumler, Ruijin Ma, and Fang Ren provided me with an unforgettable learning experience that I will always treasure, and showed unconditional patience with me and my plethora of questions. A special thanks to Mark for letting me create my own learning experiences, the many brainstorming sessions of better communication with graphics, and for not hating me after I requested a different advisor.

I'd like to thank my family and friends for their unconditional love and support while I've taken the time to find and pursue the career path that was right for me. A special thanks to my brother, Matt King, for helping me with graphics. I look forward to future collaborations with you.

One last thank you to Cohort 21. We've been through a lot this year, and I'm so thankful for each and every experience I've had with you.

Data is still King 



\begin{abstract}
Managing Marine Mammal Observations Using a Volunteered Geographic Information Approach

by

Melodi King
\end{abstract}

Traditional methods of gathering the data needed to understand human impact on marine mammals requires extensive time and resources. To reduce the burden associated with collecting and managing marine mammal observations, a geographic information system (GIS) solution was developed using a volunteered geographic information (VGI) approach. Web and mobile applications were built for the general public to submit marine mammal observations and visualize the results. The web application also includes querying and authorized download of data. Both applications consume web services published from an ArcSDE geodatabase using ArcGIS Server 10.0 . 



\section{Table of Contents}

Chapter 1 - Introduction ................................................................................. 1

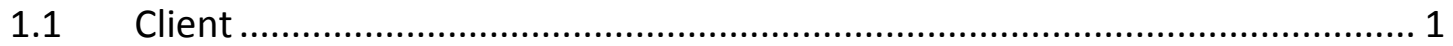

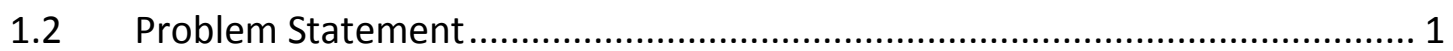

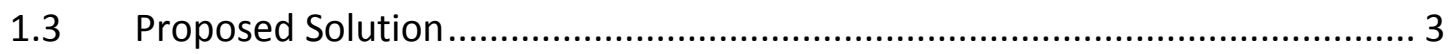

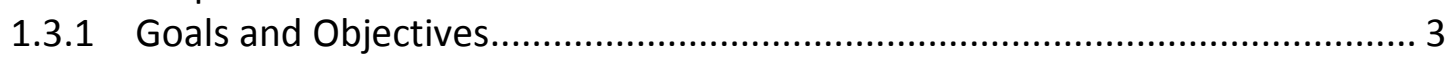

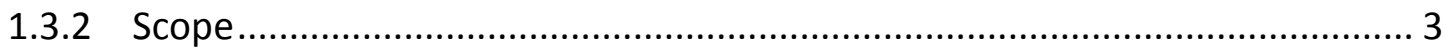

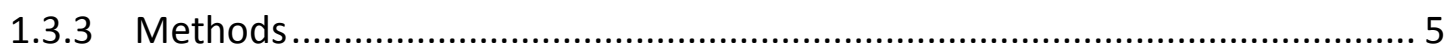

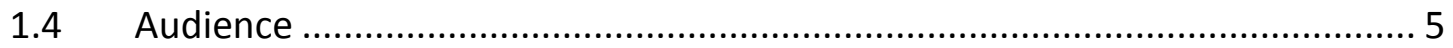

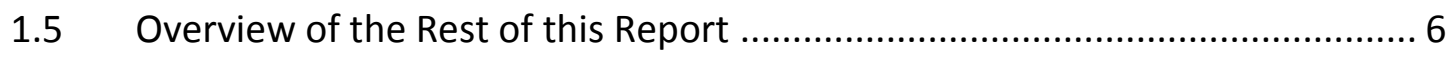

Chapter 2 - Background and Literature Review .................................................. 7

2.1 Volunteered Geographic Information and Science ...................................... 7

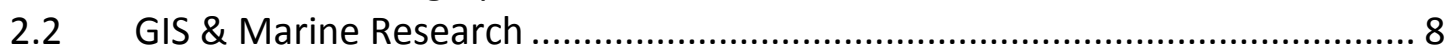

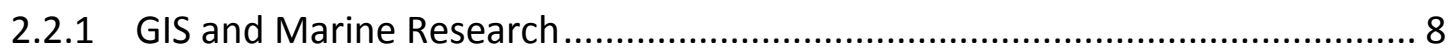

2.2.2 GIS and Spatial Analysis .................................................................... 10

2.3 Understanding Web and Mobile GIS ....................................................... 11

2.3.1 The differences between Web and Mobile GIS ........................................... 11

2.3.2 Mobile GIS Approaches...................................................................... 12

2.4 User Interface Design............................................................................ 12

2.4.1 Simplifying Task.................................................................................... 13

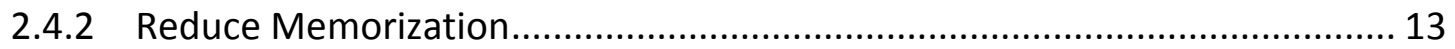

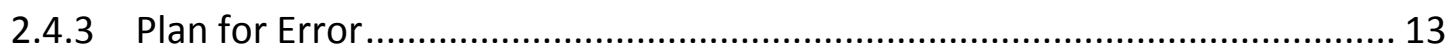

2.4.4 Testing the Usability of Map User Interfaces ............................................ 13

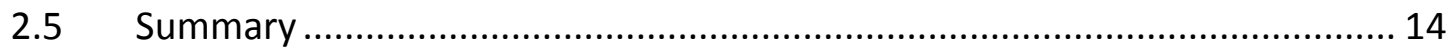

Chapter 3 - Systems Analysis and Design ............................................................ 15

3.1 Problem Statement ........................................................................... 15

3.2 Requirements Analysis....................................................................... 15

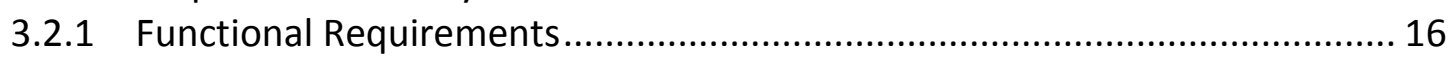

3.2.2 Non-Functional Requirements............................................................. 17

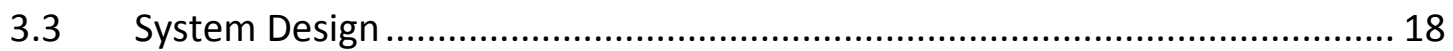

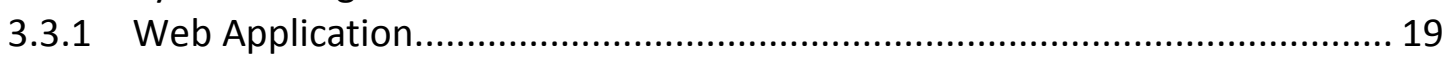

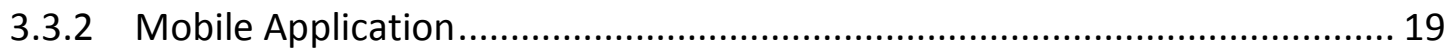

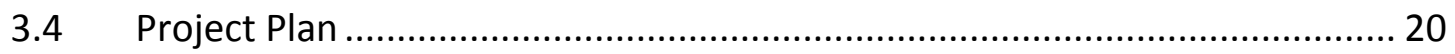

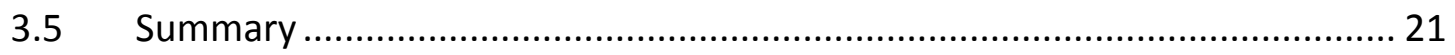

Chapter 4 - Database Design ................................................................................. 23

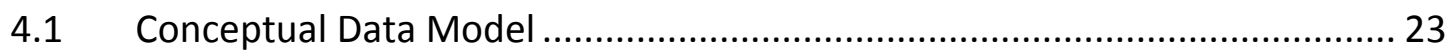

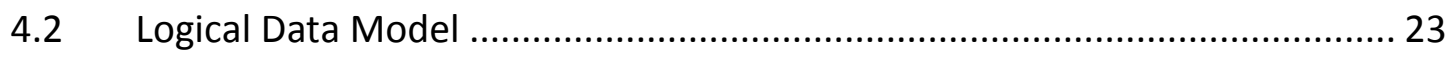

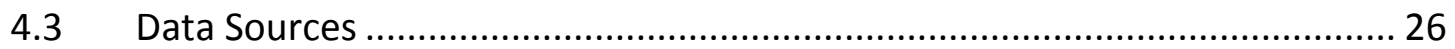

4.4 Data Scrubbing and Loading ................................................................... 27

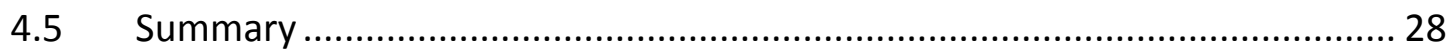


Chapter 5 - Implementation of the Web Application ........................................ 29

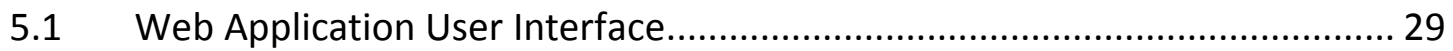

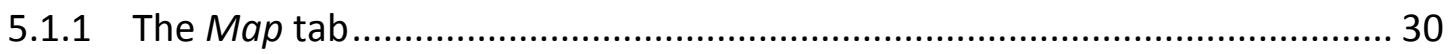

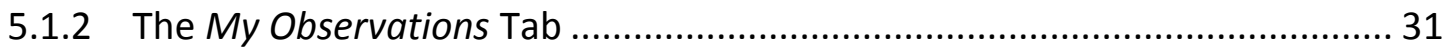

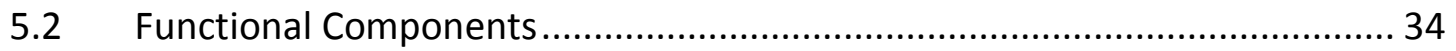

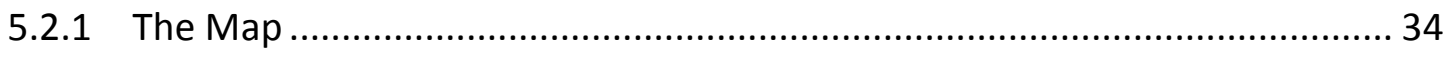

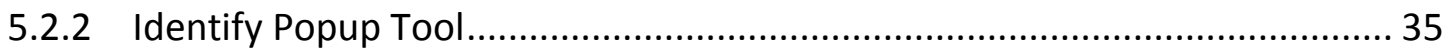

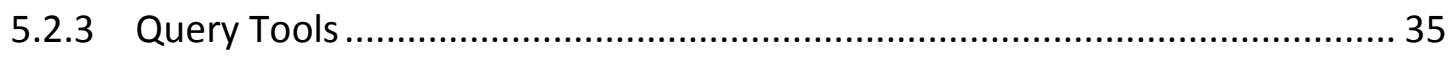

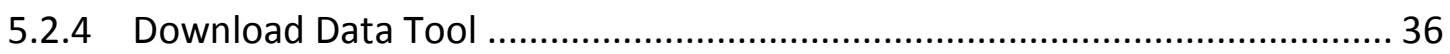

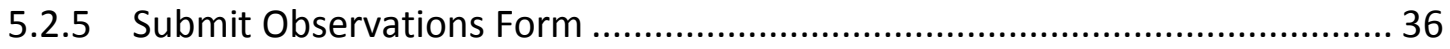

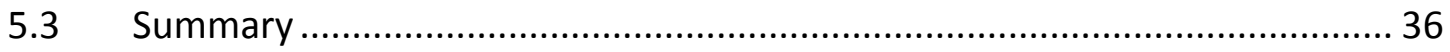

Chapter 6 - Implementation of the Mobile Application.................................... 37

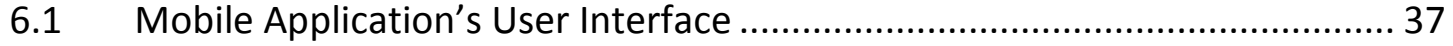

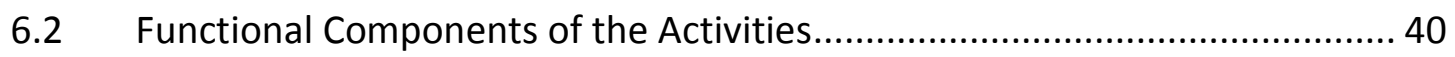

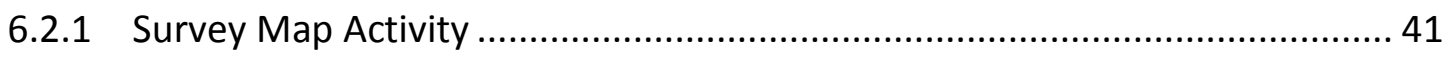

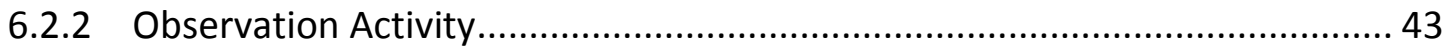

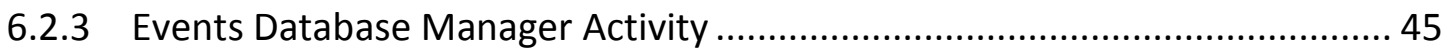

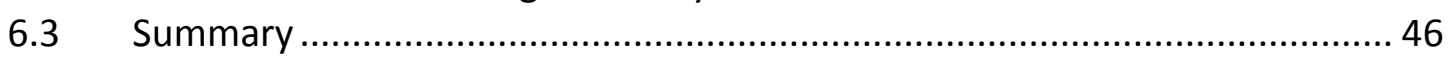

Chapter 7 - Implications of Volunteered Geographic Information Software Development 47

7.1 Considerations for Volunteered Geographic Information and Science ......... 47

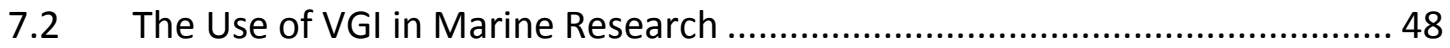

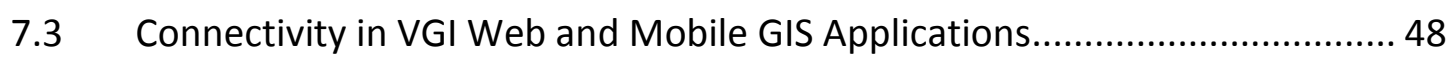

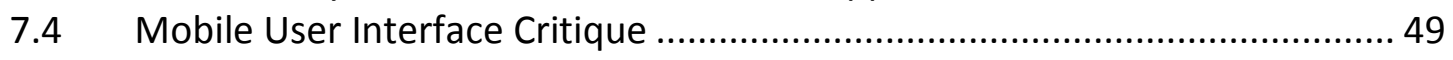

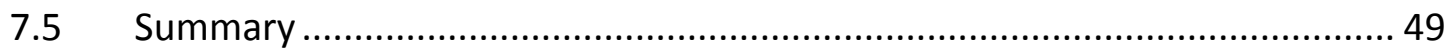

Chapter 8 - Conclusions and Future Work ..................................................... 51

Works Cited 53

Appendix A. Geodatabase Domains .............................Error! Bookmark not defined.

Appendix B. Mobile Application Activities Code ........... Error! Bookmark not defined.

Appendix C. Web Application HTML and Javascript Code .......... Error! Bookmark not defined. 


\section{Table of Figures}

Figure 1-1: VGI-approach for collecting data for a long term study of marine

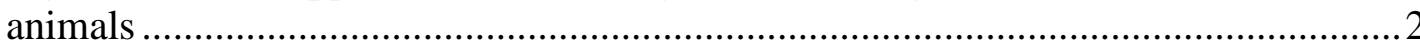

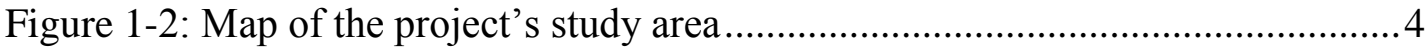

Figure 1-3: Project methods life cycle diagram ....................................................5

Figure 2-1: Common data types in the ArcMarine Data Model ................................9

Figure 2-2: Some associations found in the ArcMarine Data Model ........................ 10

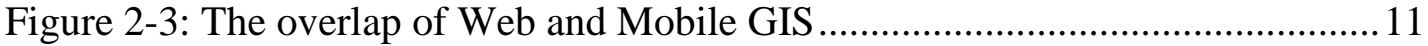

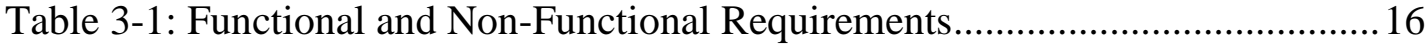

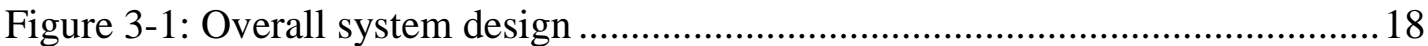

Figure 3-2: A model of the project plan..................................................................20

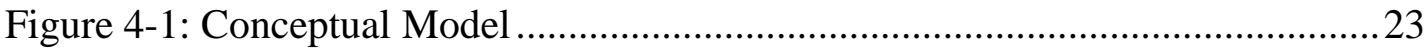

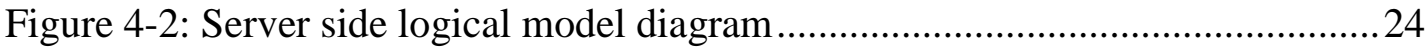

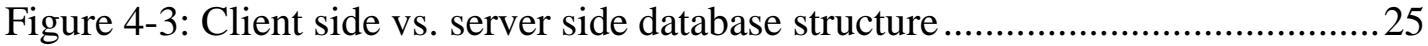

Figure 4-4: Client side logical model diagram .................................................26

Figure 4-5: Data collection sheet used by client's students and volunteers ..............27

Figure 5-1: Functional components of the web application.....................................29

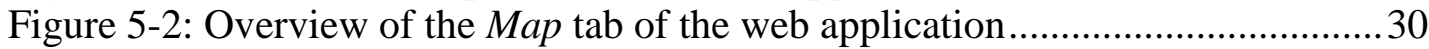

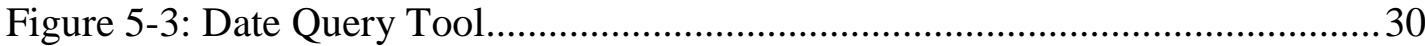

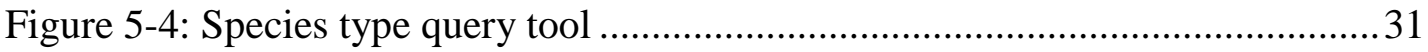

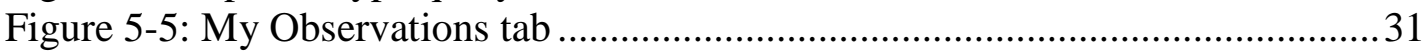

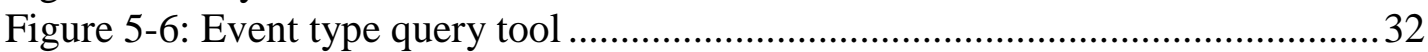

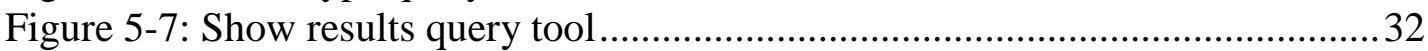

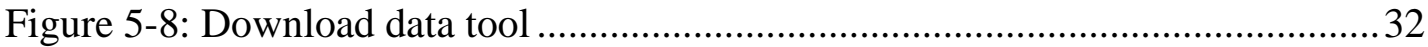

Figure 5-9: Submit observations for in accordion pane.........................................33

Figure 5-10: The use of auto-fill and drop-down use for the Submit Observations

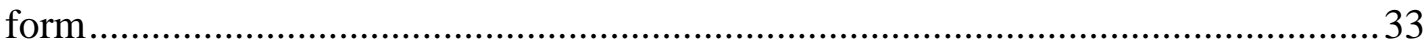

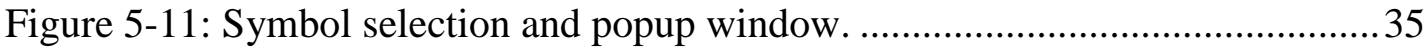

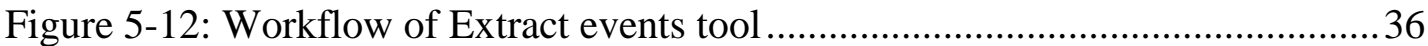

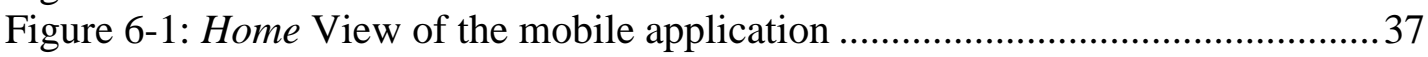

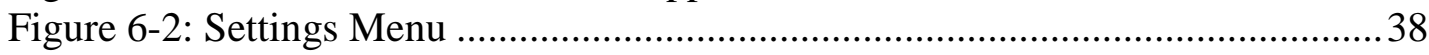

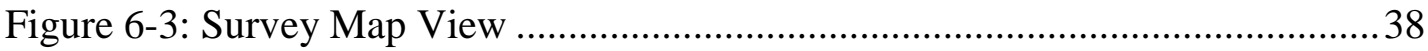

Figure 6-4: Observation View showing the required and optional components

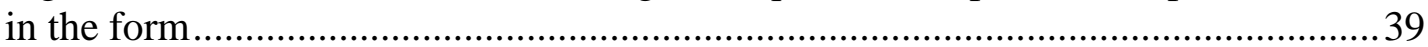

Figure 6-5: Screenshots from the dialog boxes in the Observation form ..................40

Figure 6-6: Android Life Cycle ........................................................................ 41

Figure 6-7: Functional components of the Survey Map Activity .............................42

Figure 6-8: Functional components of the Observation Activity .............................43

Figure 6-9: Detail components provided to the user upon species category

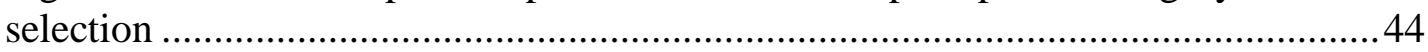

Figure 6-10: Comparison of beginning and ending of the observation form ............45

Figure 6-11: Functional components of the Events Database Manager Activity ......46 



\section{List of Tables}

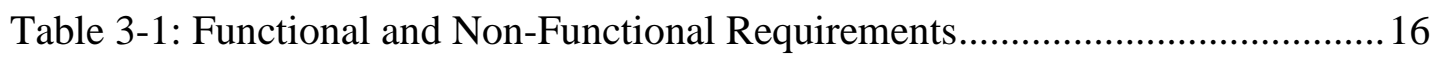

Table 5-1: Functional components and their corresponding methods .......................34

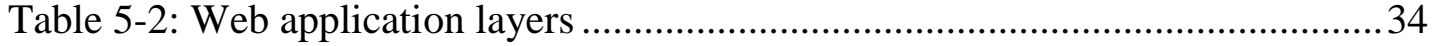





\section{List of Acronyms and Definitions}

$\begin{array}{ll}\text { API } & \text { Application programming interface } \\ \text { CINMS } & \text { Channel Islands National Marine Sanctuary } \\ \text { CWA } & \text { Coastal Web Atlas } \\ \text { EBM } & \text { Ecosystem-based management } \\ \text { GIS } & \text { Geographic information system } \\ \text { GPS } & \text { Global Positioning System } \\ \text { GUI } & \text { Graphical user interface } \\ \text { IDE } & \text { Integrated development environment } \\ \text { IIS } & \text { Internet Information Services } \\ \text { NOAA } & \text { National Oceanic and Atmospheric Administration } \\ \text { OBIS-SEAMAP } & \text { Ocean Biogeographic Information System-Spatial Ecological } \\ & \text { Analysis of Megavertebrate Populations } \\ \text { PPGIS } & \text { public participation GIS } \\ \text { SDE } & \text { Spatial Database Engine } \\ \text { SDK } & \text { Software development kit } \\ \text { SOD } & \text { Sudden oak death } \\ \text { URL } & \text { Universal Resource Locator } \\ \text { VGI } & \text { Volunteered geographic information }\end{array}$




\section{Chapter 1 - Introduction}

The oceans of the world play an indirect but fundamental role in life; they are used for activities such as shipping, procuring food, recreation, and travel. Their uses have also expanded to include renewable energy and large scale aquaculture. However, it was not until recently that scientists began establishing a scientific baseline for evaluating the health of the marine ecosystem (Ruckelshaus, Klinger, Knowlton, et al., 2008). This baseline is particularly important to recent efforts in evaluating principles for marine spatial planning for effectively managing marine resources (Foley, Halpern, \& Micheli, 2010).

In order to understand behavior of, and human impact on, marine mammals, extensive manpower for collecting and processing data is required, due to the complex interactions between marine mammals, humans, and oceanic processes. The burden associated with this type of research can be reduced using a volunteered geographic information (VGI) approach with a geographic information system (GIS). Incorporating familiar user interfaces, such as web and mobile applications, allows researchers to spend more time performing analyses while simultaneously encouraging awareness and environmental stewardship in users.

This chapter was designed to introduce the reader to the project. Section 1.1 introduces the client. The second section, 1.2, defines the problem addressed. The proposed solution, including the goals and objectives, scope, and methods are discussed in Section 1.3. The fourth section, 1.4, outlines the target audience for this report. Finally, Section 1.5 sets the expectations for the remainder of the document.

\subsection{Client}

Dr. Lei Lani Stelle is a biology professor at the University of Redlands whose research pertains to the human impacts on marine mammals and their habitat use. Specific components of her research include evaluating marine mammal species' associations, determining swim paths and behaviors, assessing vessel-induced injuries, and understanding energy expenditures of marine mammals during a migration path affected by human interactions.

Over the course of the project, Dr. Stelle was responsible for describing the types of data that were collected and verifying that the system design met her needs. Additionally, she was responsible for approving the user interface design of the web and mobile applications during the testing and discussion tasks of the project's life cycle. Finally, the client acted as a domain knowledge expert when questions arose during design and development of the solution.

\subsection{Problem Statement}

The challenge that the client faced was how to generate a high volume of quality data for her long-term study on marine mammal migratory behavior and human impact. In order to reduce the cost and effort required in the data collection process, Stelle decided to 
explore a VGI approach to collecting this data, which incorporates the collection of data by both researchers and members of the general public (Figure 1-1).

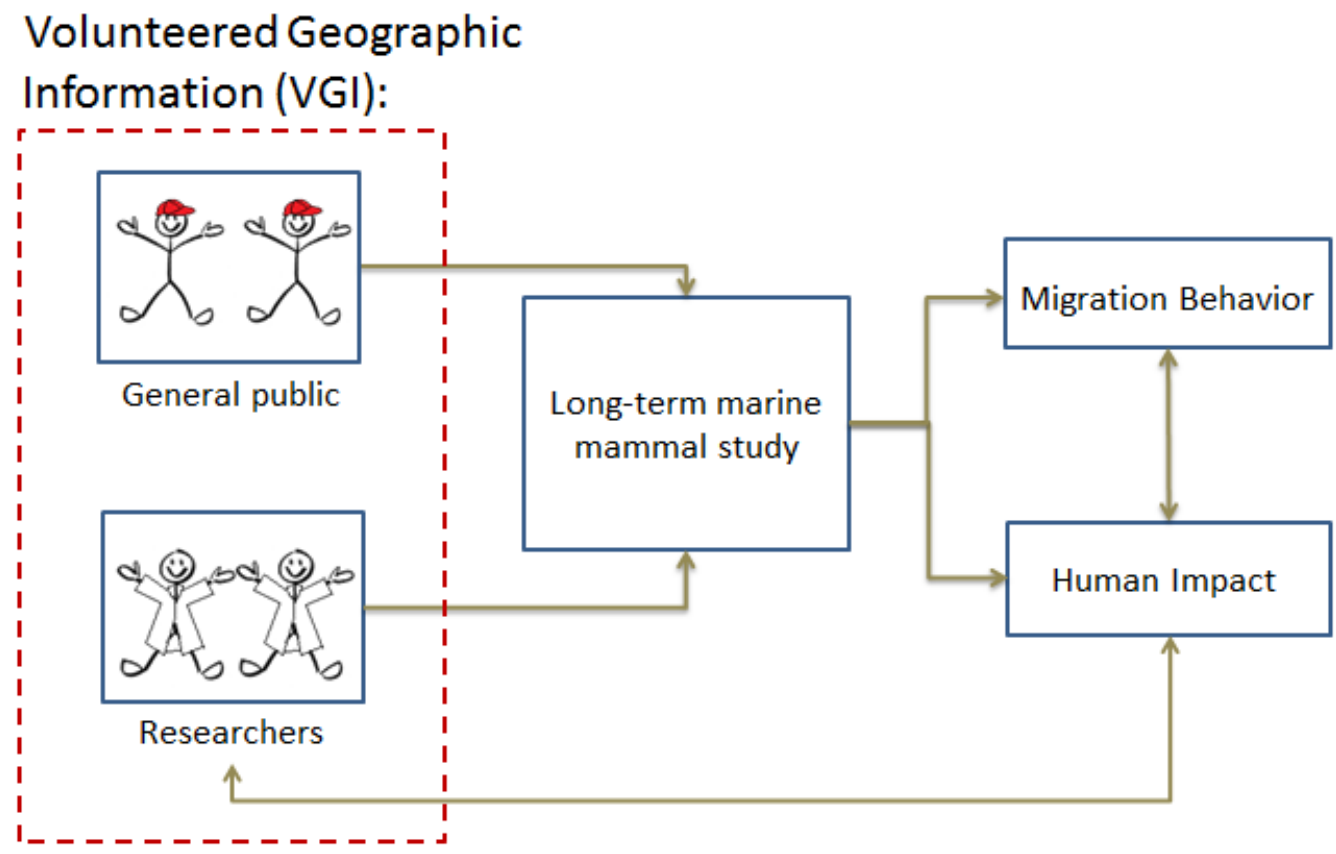

Figure 1-1: VGI-approach for collecting data for a long term study of marine animals

Currently Stelle works with volunteer citizen scientists through Earthwatch, an organization that facilitates participation by members of the public in the scientific process. Additionally, Stelle collects data with undergraduate students performing research for thesis projects. She believes that by including the general public in the data collection process she will further reduce difficulties in maintaining long-term studies while simultaneously encouraging awareness and environmental stewardship.

Although including the general public in the data collection process would increase the amount of data collected, it would also introduce questions of data quality. The client has very few protocols in place for ensuring high data quality from each of the sources. For example, currently, data are hand-written, leaving room for error during the process of transferring them from paper to electronic source. Without continuity and consistency in the data collection process, an unnecessary amount of time has to be spent preparing and organizing data over the course of a long-term study.

With the approach developed in this project, Stelle would be able to focus efforts on understanding the relationships between migrating mammals and humans, in addition to improving the learning experience for the students and volunteers she works with. Specifically, because of the nature of GIS databases, data collected by volunteers will have continuity and consistency. Having a centralized database that can be queried would allow Stelle to collaborate with other researchers. Additionally, having the data stored in spatial tables allows her to ask advanced spatial questions. 


\subsection{Proposed Solution}

After careful review of previous work and consideration of the client's requests, a solution was proposed. The following section outlines the proposed solution and its appropriateness for the client. It includes a discussion of project goals and objectives, scope, and methodologies used to develop the solution.

\subsubsection{Goals and Objectives}

The two problems addressed in this project provided the client with geographic workflows for managing and sharing her data. The first problem was how to generate a volume of quality data for a study on marine mammal migratory behavior and human impact. This problem was solved using a VGI-based strategy which incorporated the general public in the data collection process to increase the amount of data being collected. More specifically, mobile and web application prototypes were developed that can be used by volunteers, researchers, and members of the general public to submit marine mammal observation data. Web and mobile technology were chosen because they were already demonstrated to be successful in a VGI-based study (Connors, Lei, \& Kelly, 2011). This solution decreased the amount of time researchers and volunteers spent in the field and increased the amount of data collected. It also provided the client with the data necessary to perform her research.

The second problem addressed in this project was the data management methods. The client stored data in Microsoft Excel, Access, in species-specific programs, and in programs developed for specific projects. Without proper management of the data, a large amount of time was spent on organization efforts and preparing the data for analysis.

This problem was solved with the development of a centralized geodatabase used to manage data submitted from student and professional researchers, volunteers, and the general public. The geodatabase was used to house marine mammal observations. The outcomes of the solution were time savings to the client and possible identification of marine mammal observation data standards. The development of a geodatabase allowed Stelle to spend less time preparing data for analysis, more time improving the learning experience for her students and volunteers, and collaborating with her colleagues in defining a baseline for evaluating the health of the marine ecosystem.

\subsubsection{Scope}

While the purpose of this project was to demonstrate the feasibility of using a VGI approach to managing marine mammal observation, only a very basic, but extendable, solution was developed. The scope included the development of web and mobile applications that allows the user to submit and visualize their observations. The solution also included a geodatabase for storing the submitted observations.

The Spatial Database Engine (SDE) geodatabase, a type of relational database management system, was developed for use with the ArcGIS Server 10.0. The geodatabase was designed to hold observation data and corresponding evidence and to tie observations to the user who submitted them, through the use of usernames. However, the proof of concept was designed so that users had the same level of access. There were no 
database permission differences between the experts (researchers, students) and general public users.

Due to the time constraints of the project, the scope of the database development was limited and client expectations were clearly defined. The geodatabase's schema was developed and its capability was demonstrated with sample data. However, cataloging of the current data was the client's responsibility. Additionally, the client was responsible for describing the types of data that were collected and verifying that the geodatabase's schema met their needs.

The web application was developed to allow users to visualize, query, and download data. Specifically, it was designed to allow users to visualize and query the entire database by date and species type. Upon logging in, the application allowed users to query by event type (observation, track update) and choose to visualize all of the data in the database or only their data. The web application was also developed to allow users to download data upon log in to the system. The users' identity was not verified when logging into the system with this initial concept. Finally, forms were developed to allow users to enter data that they collect in the field.

The mobile application's functionality was limited to data submission and visualization. While there were several platforms for which the mobile application could be developed, it was only developed for one. The mobile application was designed to store collected data locally, in addition to syncing the data with the geodatabase on the server. The client was asked to help design the appearance of the mobile application.

The solution was designed for use between Newport Beach and Long Beach (Figure 1-2) in southern California. The project was assigned a spatial scope for multiple reasons. Keeping the region focused resulted in a small list of species native to the region that the user would need to choose from. This small area also had reliable connectivity to the mobile network, which supported an environment to test the data syncing.

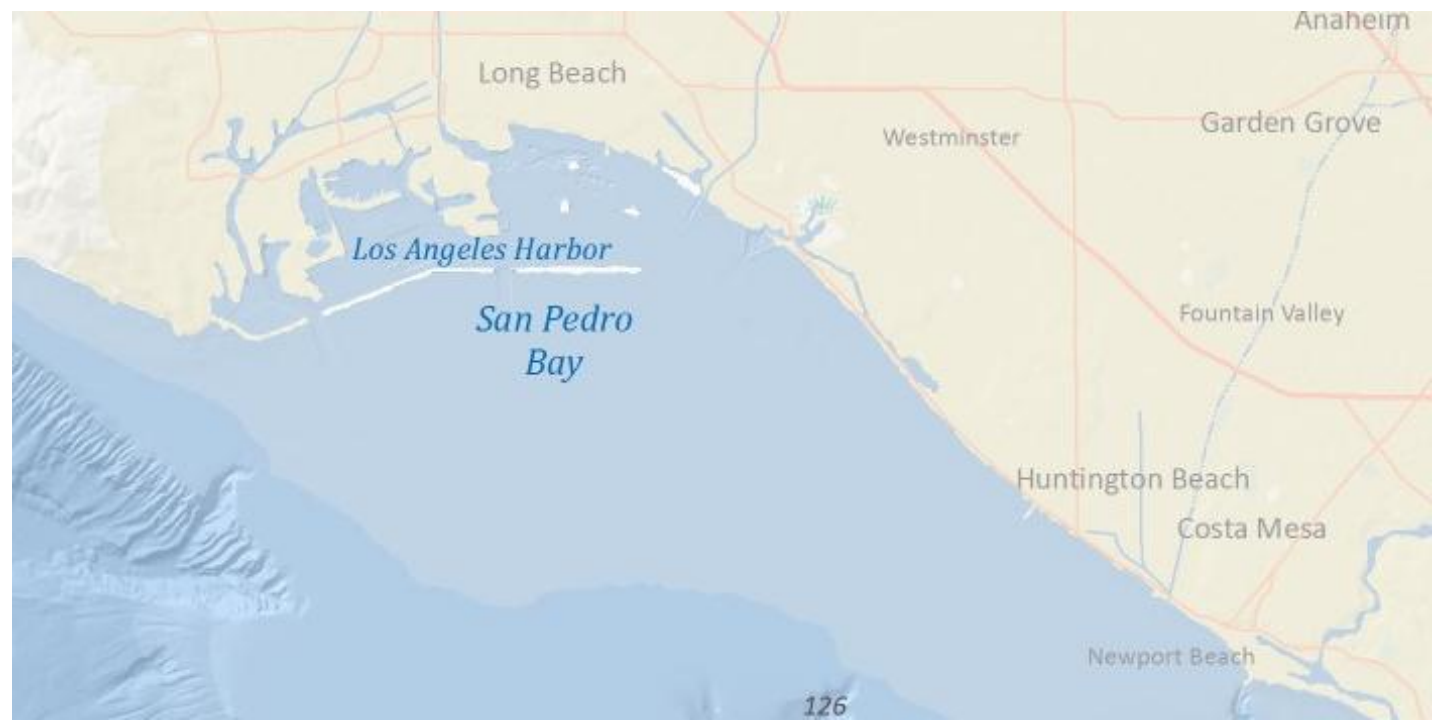

Figure 1-2: Map of the project's study area 


\subsubsection{Methods}

The project was split into three different packages: the geodatabase, the web application, and the mobile application. Each package went through a staged developed life cycle (Figure 1-3).

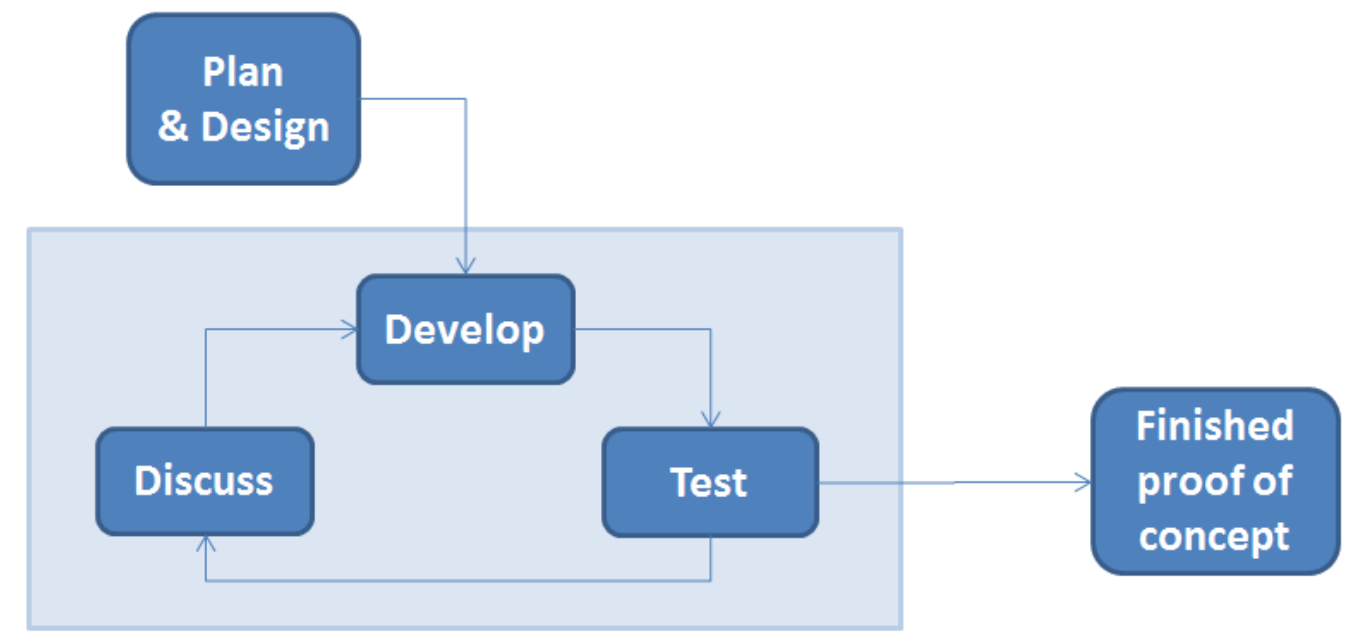

\section{Figure 1-3: Project methods life cycle diagram}

During the plan and design phase for the geodatabase, requirements were classified into functional and nonfunctional requirements. Conceptual model and logical models were then designed. In the development phase an SDE geodatabase was created. Additionally, map and feature services were published using ArcGIS Server 10.0 for consumption by the web and mobile applications. The logical model went through test and discussion phases before the finished proof of concept was reached. Each time there was a change in the database's schema, changes were made to a small set of test data.

During the plan and design phase for the web and mobile applications, the appropriate technology for development was decided. The web application was developed using Esri's ArcGIS Application Programming Interface (API) for Javascript and the mobile application was developed using Esri's Android Software Development Kit (SDK). During the develop, test, and discussion phases of the web and mobile applications, each of the functional requirements was built and pieced together to get the final proof of concepts. The develop phase for both of the applications included researching the APIs' classes and corresponding methods.

\subsection{Audience}

The intended audience for this report includes individuals who have an introductory knowledge of GIS, have a basic understanding of programming concepts; are interested in data collection using a VGI approach, or are interested in data collection techniques for marine mammal research. No specific knowledge of ArcGIS or programming is assumed. 


\subsection{Overview of the Rest of this Report}

The remainder of the report describes how the project components were implemented.

Chapter 2 provides a literature review of relevant topics. Chapter 3 describes the system design of the project. This is followed by a discussion of the database model in Chapter

4. Chapter 5 refers to the implementation of the web and mobile applications. Chapter 6 describes the lessons were learned during the software development process and the analysis that can be done with the data collected. The report closes with Chapter 7, a conclusion and discussion of future work. 


\section{Chapter 2 - Background and Literature Review}

A literature review was performed during the project planning phase, during which four relevant topics were chosen. Because of the Volunteered geographic information (VGI) nature of the project, Section 2.1 is dedicated to understanding its use in web and mobile GIS as a means of collecting data. Section 2.2 presents the use of GIS in marine research. Section 2.3 discusses the differences between web and mobile GIS technologies and their appropriate applications. Designing user interfaces that can reach a broad audience is important in VGI. Because of this, user interface design is discussed in Section 2.4. The chapter is concluded with a summary in Section 2.5.

\subsection{Volunteered Geographic Information and Science}

Volunteered geographic information is closely tied to citizen science, which is the involvement of interested members of the public in parts of a scientific project such as data collection and analysis. It has been utilized in applications such as recording bird observations (The Cornell Lab of Ornithology, 2011) and online game playing in understanding protein folding (UW Center for Game Science, 2011). Allowing citizens to participate in the scientific inquiry process may bring about awareness, empowerment, and stewardship. Additionally, the inclusion of citizen scientists may help reduce the gaps that have historically divided the public, researchers, and policymakers in environmental management efforts (Connors, Lei, \& Kelly, 2011).

A similar concept is public participation GIS (PPGIS). PPGIS is strongly focused on engaging citizens in the sustainability of their communities. "It is an interdisciplinary research, community development and environmental stewardship tool grounded in value and ethical frameworks that promote social justice, ecological sustainability, improvement of quality of life, redistributive justice and nurturing of civil society," (Aberley \& Sieber, 2002).

Goodchild (2007) coined the term volunteered geographic information to describe geographic data provided voluntarily by individuals. The development of Web 2.0, Global Positioning System (GPS), and the rapid assimilation of mobile technology made VGI practical. Web 2.0 resulted from the development of protocols that made the communication between user and server a two-way conversation. This enabled users to create and edit information stored on the servers through the browser interface. In the 1980s the GPS was developed. Originally created for military purposes, the GPS made its way into the hands of the public around 1990. GPS allows for quick and easy direct measurement of locations on Earth and has been used in a wide variety of applications (Goodchild, 2007).

The use of mobile and web applications that utilize a VGI-based strategy in the collection of data in long-term environmental studies is a relatively new field. There is a working prototype of this data collection method called OakMapper, which was developed at University of California at Berkeley. OakMapper is a mobile (iPhone) and web-based effort to encourage the public in monitoring the sudden oak death (SOD) of oak trees in California caused by the ramorum leaf blight (p. ramorum) virus (Geospatial Innovation Facility, 2012). 
Advancements in web and mobile GIS technologies that utilize a VGI strategy for scientific data collection are limited. Glennon (2011) created the Geyser Notebook application for Android. The application allows users to view information about the Yellowstone geysers and report eruption observations. User accounts are created, but there appears to be no differentiation between a researcher and a member of the general public. Within Geyser Notebook, a timeline shows observations from all users, and "my reports" shows only the user's observations. In addition to a mobile application, a web application has been developed that allows users to view the data stream (Glennon, personal communication, 2012). However, there is no evidence that this application is utilized by researchers in understanding geyser activity in Yellowstone National Park.

\subsection{GIS \& Marine Research}

The complex nature of marine studies poses a unique challenge for researchers and decision makers. "Traditional management strategies, which focus on individual sectors of coastal ecosystems, such as managing single species habitat, or areas, have failed to address these intricate relationships between humans and coastal ecosystems," (Bauer, 2012). More integrated and comprehensive management strategies, such as Ecosystembased Management (EBM), are being developed to address this problem (Jones \& Ganey, 2009). Geographic information systems are being used in data collection and management in addition to analysis relevant to marine research.

\subsubsection{GIS and Marine Research}

Several efforts have been made to make data collected more widely available to researchers and decision makers. The Ocean Biogeographic Information System Spatial Ecological Analysis of Megavertebrate Populations (OBIS-SEAMAP) is a spatially referenced online database, aggregating marine mammal, seabird and sea turtle observation data from across the globe and have aggregated data since 2002. The OBISSEAMAP uses of geospatial web feature services. Ideally, "this makes data easy to use by modelers in a scientific workflow," (Best, et al., 2006).

The OBIS-SEAMAP is just one example of geospatial web services being used by researchers and decision makers in the field. Coastal Web Atlases (CWA) are increasingly popular web-based tool. The California Coastal Atlas was initiated in 1993. Its primary goals are to create a platform for sharing high quality coastal data, provide a medium for information sharing between scientists and public policy makers (University of Washington Sea Grant Institude, 2011). Additionally, the National Oceanic and Atmospheric Administration (NOAA) Coast Watch has developed a browser for downloading contour, grid, and vector datasets. The data available include: currents, chlorophyll, sea surface temperature, and wind stress to name a few (NOAA, 2011).

While sites like OBIS-SEAMAP, California Coastal Web Atlas, and NOAA provide a wide range of uses, there are also organizations that have made a more focused effort of collecting and sharing data. Oregon State University has made chlorophyll and temperature data available through an Ocean Productivity website (O'Malley, 2010). Although the data isn't available for download and there is no mapping component, the Channel Islands National Marine Sanctuary has a website in which users can submit marine mammal observations (National Ocean Service, 2011). 
Essential to data collection and sharing is the data storage. The use of geospatial web services provides a method for accessing and downloading data stored on servers, but they do not address the need for best practices for storing data collected in the field and data downloaded from web services. Geodatabases provide components, such as subtypes and domains, to better manage spatial and non-spatial data.

In addition to the geodatabase are data models. Data models are schemas for organizing groups of relevant information. The Marine (also known as ArcMarine) data model available from Esri, is one of the more widely recognized data models for marine phenomena. The data model can be used to store several common data types: tables, marine points, marine lines, and marine areas (Figure 2-1).

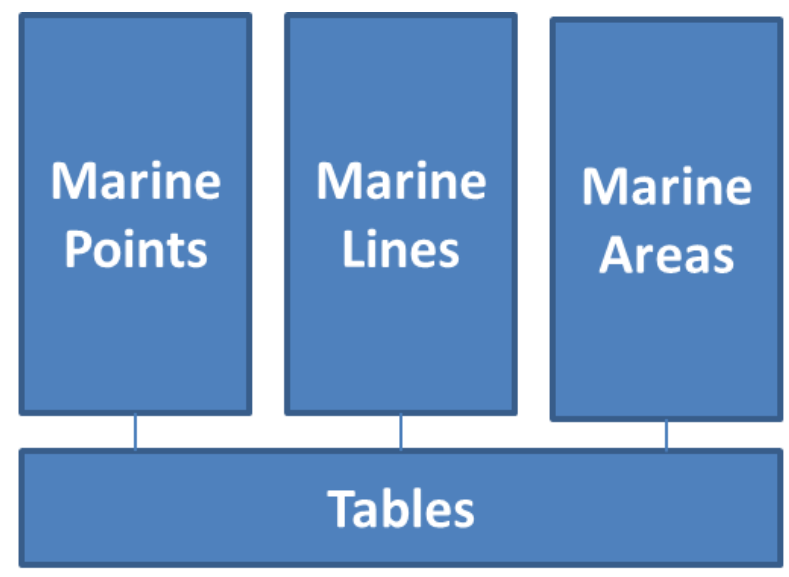

Figure 2-1: Common data types in the ArcMarine Data Model

The tables are intended to hold non-spatial elements. These elements are associated with one or more of the three spatial data types. Some of the associations are illustrated in Figure 2-2 between table elements and marine points and between table elements and marine lines. To demonstrate, a vehicle (non-spatial) such as a boat records a run line (spatial) when it is out on a trip. Similarly, a survey info (non-spatial) record can have one or more survey points (spatial) associated with it. 


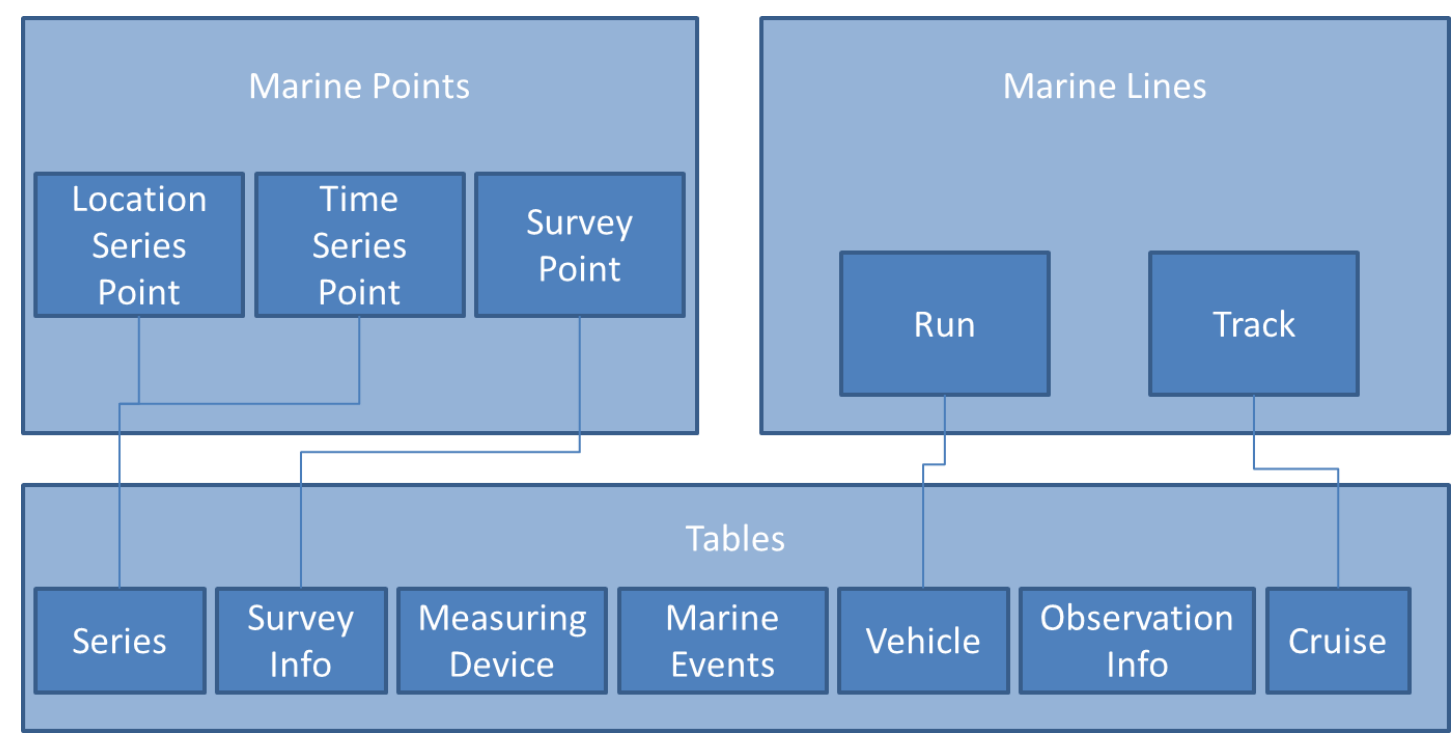

\section{Figure 2-2: Some associations found in the ArcMarine Data Model}

Whether data is collected in the field or downloaded from geospatial web services a data model, such as ArcMarine, is essential to managing the data. ArcMarine was designed to allow users access to analysis capabilities of GIS (Wright, Blongewicz, Halpin, \& Breman, 2007). However, the data model is normalized and is not an ideal model for web and mobile projects. Additionally, the data model doesn't include a table associating the user who submitted the data. This demonstrates that it was not intended for storing volunteered geographic information.

\subsubsection{GIS and Spatial Analysis}

Once data has been collected and stored in a GIS, analysis can be performed to understand a wide array of topics. These topics include, but are not limited to human impact, and density and abundance. Some of the GIS techniques used to understand these topics include spatio-temporal analysis to understand patterns in the data and overlay techniques to understand human impact in marine mammals.

The use of space in understanding human impact is by no means a new idea, but it has become increasingly popular in the marine world since 2000. Since then, it has been used in a variety of applications. In 2008, Halpern et al. published a paper on research assessing the human impact on marine ecosystems. The study included several indicators relevant to fishing, pollution, and climate changes that were evaluated at a global scale. Raster analysis was performed to calculate and cumulate human impact indicators. The results found that there were virtually no ecosystems unaffected by humans. While this research is extremely valuable, similar research is needed at the local and regional levels.

Another research group recently released two studies using similar overlay and raster analysis techniques applied directly to marine mammals. One was directed at finding geographic ranges and patterns of richness and composition in an attempt at finding potential conservation sites (Pompa, Ehrlich, \& Ceballos, 2011). Their second study was directed at identifying which species were at greatest risk and where the risk is 
globally (Davidson, et al., 2012). A discussion of their findings found that more local data on migratory routes, locations of feedings, and calving/pupping could be used to produce better results. These are the exact types of data that this proof of concept was designed to collect, if fully deployed.

The preceding examples demonstrate the use of GIS in marine mammal research, and how it improves our understanding of a variety of topics. A need for larger scale assessment of human impact on marine mammals was revealed.

\subsection{Understanding Web and Mobile GIS}

Web and Mobile GIS are two different media for handling geographic information systems. The method of consumption is essentially the only difference between them. Web GIS applications are accessed through a browser on desktops and laptops. On the other hand, Mobile GIS applications are accessed through smart phones and tablets. However, advances in wireless communication have allowed users to access web-based GIS applications with both smart phone browser technology and native applications. This has caused significant overlap in the Web and Mobile GIS fields (Figure 2-3).

\begin{tabular}{|c|c|}
\hline Web & Mobile \\
\hline Connected & canConnect \\
\hline Shares & canShare \\
\hline hasLocation & $\begin{array}{c}\text { hasLocation: } \\
\text { LocationChanges }\end{array}$ \\
\hline cloudStorage & localStorageBuffer \\
\hline & hasOrientation \\
\hline
\end{tabular}

Figure 2-3: The overlap of Web and Mobile GIS

\subsubsection{The differences between Web and Mobile GIS}

There are significant advantages of Mobile over Web GIS. However, there are still significant technical challenges that give Web GIS an upper hand. Mobile GIS technologies can replace existing field paper-based workflows (Fu \& Sun, 2011). Mobile GIS also provides a highly mobile environment that can be accessed by several users. Modern mobile devices are equipped with a GPS chip and other hardware devices that can reduce the amount of equipment needed in field work. Finally, Mobile GIS also enable users to work in a disconnected environment in which they have no access to internet or mobile services.

However, limited wireless communications also pose a technical challenge for the use of Mobile GIS. The desktop and server machines that are used in Web GIS provide more powerful CPU, memory, and battery power (Fu \& Sun, 2011). Mobile GIS is also 
limited in its screen size and keyboard size. This can be particularly inhibiting to field workers uncomfortable with mobile devices and in poor environmental conditions. There are significant advances of Mobile GIS over Web GIS. But because there are still some significant technical challenges, Web GIS still provides benefits.

\subsubsection{Mobile GIS Approaches}

The overlap in Web and Mobile GIS has resulted in two different approaches to developing Mobile GIS applications: native-based and browser-based. Native applications are those that are designed to run on a device's operating system, such as Apple iOS or Google Android. This forces the application developer to adapt the application depending on the operating system's platform language and operating system.

The two popular platforms are iOS using the Objective-C language and Android using Java. Objective-C is an exclusive language that isn't as commonly used (Viswanathan, 2012), while Java is a commonly used language and has extensive documentation for its SDK (Google, 2012). Java's large programming community has resulted in a reliable online resource community, easing the programming and cost burden for the project developer. Finally, phones with an Android operating system have become increasingly popular (Lloyd, 2012).

When developing a mobile application for Android devices, the developer must decide which operating system to develop for. To date, Gingerbread (2.3.3) is the predominant operating system on all Android platforms (Android 2.3.3 APIs, 2012). In the past eight months, all Android phones users had the option to switch to a newer operating system (IceCream Sandwich: 4.0.4) on their phones. However, eight months after IceCream Sandwich's release, only 10\% of users had upgraded (Aguilar, 2012). This demonstrates that the developer must decide on both a platform and an operating system when creating mobile applications.

Just like many Web GIS applications, mobile browser-based applications can be built to work with a plugin, such as Flash Lite or Silverlight mobile, or with html and JavaScript (Fu \& Sun, 2011). HTML browser-based applications can be developed once for a wider device range using a single language. This makes deployment of those applications across several platforms much easier. These types of applications also don't have to be purchased through application stores.

Unfortunately, HTML browser-based do not have full, and easy, access to the hardware on mobile devices, whereas native applications do. Additionally, because browser-based applications require connectivity, they cannot be used in disconnected environments. On the other hand, native applications can use local storage to work in such environments.

\subsection{User Interface Design}

Graphical user interfaces (GUI) have come to be an expected part of the experience for most computer users. When initially being developed, Apple Desktop Interfaces made two basic assumptions, "that the user can see, on a computer screen, what they are doing; and they can point at what they see," (Apple, 1987). Designing a good user interface requires the implementation of principles that have been repeatedly proven to be

effective. Several sets of principles and rules of thumb have been put together by subject 
matter experts, some of which include: simplify the structure of tasks, minimize memorization, plan for error, and know the user.

\subsubsection{Simplifying Task}

User Interfaces often provide new workflows for users to complete tasks that they were already doing using different methods. Asking users to change the way they currently perform a task can be difficult. In his book, The Design of Everyday Things, Donald Norman provides three technical approaches for ensuring success that are particularly relevant. The first is "Keep the task much the same, but provide mental aids." Mental Aids such as sticky notes and alarm clocks are simple examples of this. The second approach is, "Use Technology to make obvious what would otherwise be invisible." This approach can be implemented by giving the user feedback and allowing them to monitor the state of the system they are interacting with. The third approach is to "change the nature of the task." Having a thorough understanding of how the current workflow of a task operates, allows the designer to alter the way in which users are asked to provide input. Changing the nature of the task can make difficult tasks seems less daunting.

\subsubsection{Reduce Memorization}

There are several methods for reducing the memorization expected of the user. One method is to use a see-and-point method over remember-and-type (Apple, 1987). This can be implemented with the use of dropdown menus for making selections rather than text boxes for users to fill out. Another method is to use real-world metaphors. Doing this allows "users to transfer knowledge of how things should look and world," (Mandel, 1997). Using knowledge that is both in the world and in the head of the user, can make their experience with the user interface faster and more efficient (Norman, 1988).

\subsubsection{Plan for Error}

It is always safe to assume that the user will make mistakes in any number of ways when interacting with the interface. One method for handling this is to allow the user to recover if mistakes are made (Norman, 1988). This can be done by asking users to confirm actions before completing them and allowing users the permission to undo and redo actions.

\subsubsection{Testing the Usability of Map User Interfaces}

Understanding the usability of an interface is extremely important in its design and development. A direct method for determining whether or not the interface design is appropriate for the user is to test its usability. This is particularly important in user interfaces that are being designed for a broad audience, such as applications involving maps and the general public. A blog was released on mapbrief.com by Brian Timoney on how the public interfaces with local government web maps. The blog was based on research performed by a GIS Analyst with the City of Denver. There were a couple of findings they were particularly relevant. The first was that people rarely changed default settings, this includes the default basemaps. Giving the user too many options may 
overcomplicate a normally simple task. The analyst also found that using an autocomplete in the map's search box drove clean queries (Timoney, 2012).

A study was recently performed on the usability of a citizen science web application. The researchers found that users had a difficult time understanding the concepts of layers in the map's legend, and that layers could be turned on and off (Newman, Zimmerman, \& Crall, 2010). This demonstrates the importance of knowing the user and the power of the real world metaphors method to engage them.

\subsection{Summary}

This chapter reviewed the background information relevant to the project. Section 2.1 discussed VGI in Web and Mobile Applications. VGI is a relatively new field, and its uses in scientific research are extremely limited. Section 2.2 talked about the GIS-based methods used in the marine field for sharing and storing data. Geospatial web services are being used to share data at large and small scale, and the use of geodatabases and data models can be useful is organized data for analysis. The use of GIS in marine spatial analysis was also discussed in this section. There is a need for large scale assessment of human impact on marine species and their environments. Section 2.3 described the differences between web and mobile GIS and why each of them plays an important role. While mobile GIS simplifies field-based workflows and can be accessed by a wide audience, web GIS solutions do not have memory, battery life, or connectivity issues. Section 2.4 discussed user interface design principles and demonstrates the importance in understanding and applying them to web and mobile applications is extremely important. 


\section{Chapter 3 - Systems Analysis and Design}

This chapter discusses the analysis of the system design of the proposed solution. More specifically, it includes a Section 3.1 which revisits the problem statement. The problem statement was used in a requirements analysis. The results from this are presented in Section 3.2. The requirements analysis was used to develop a system design and project plan. The proposed system design and the plan for its implementation are described in Section 3.3 and 3.4. The chapter is wrapped up in a summary section.

\subsection{Problem Statement}

The challenge that the client, Dr. Lei Lani Stelle, faced was how to generate a high volume of quality data for her long-term study on marine mammal migratory behavior and human impact stored in a well-organized fashion for analysis. In order to reduce the cost and effort required in the data collection process, Stelle decided to explore a VGI approach to collecting this data, which incorporates the collection of data by both researchers and members of the general public.

\subsection{Requirements Analysis}

Functional requirements describe the information and answers that the system will provide to its end users. For example, a functional requirement of the system was to allow users to submit marine mammal sightings from both a mobile device and a website. On the other hand, the non-functional requirements describe the way in which the system should perform and includes technical, operational, and transitional requirements. The technical requirements describe both the technology the client will need to maintain the system and the technology the end user will need to access the system. The operational requirements include the day-to-day or periodic maintenance requirements that the client will be responsible for to keep the system up and running. Finally, the transitional requirements are those needed for handing the system over to the client and end-users, such as training or usage documentation. The following subsections describe in detail the functional and non-functional requirements of the system that are outlined in Table 3-1 below. 


\section{Table 3-1: Functional and Non-Functional Requirements}

\begin{tabular}{|l|l|}
\hline \multicolumn{1}{|c|}{ Functional Requirements } \\
\hline Store survey logs and corresponding events, including sightings and their corresponding evidence \\
(such as photos), and position updates
\end{tabular}

\subsubsection{Functional Requirements}

There were several functional requirements of the system, as outlined in the table above. The system had to be capable of storing survey logs from a user's trip and all of the events that occurred in a single trip. There were two types of events that occurred during a trip. The first was a position update, which included a time and position. The second was an observation (or a sighting). Sightings consisted of time and position, but they also had information about the species observed and relevant information. This included a confidence rating on the data being submitted. The system was designed to classify all incoming records by subtype. If the incoming event was a sighting, the observation is classified as one of four marine mammal categories. If the incoming event was a position update, it gets recorded accordingly.

The system allowed users to submit the above mentioned survey logs and corresponding events using the web and mobile applications. This was done by developing a form that could be accessed by both. The form was developed to allow users to associate a photo with the observation being submitted. The data associated with the events are stored locally on the mobile device and the events are submitted to the geodatabase through the use of feature services. This was done using a creating a table in a SQLLite database on the device itself. 
The events submitted can be associated with the user that enters them. In the web application, the user must log in before submitting any observations. The username is temporarily held so that it can be used to tie observations with the user that recorded them. On the mobile device, the user can associate their data with an email addresses on their phone. Alternatively, by default, all data is associated with an "anonymous" user account.

The web and mobile applications were developed to allow users to visualize recorded observations and position updates. Visualization on the web application was made possible through the use of map services that access the data in the geodatabase. The events recorded on the mobile device are rendered locally from the data stored on the device rather than through map services. The feature services are only accessed during the submission of an event from the phone.

There were also functional requirements specific to the device. Additional core requirements for the web application were that users had to be able to query and download data. Querying of the data was made possible through the use of toggle buttons and a date range that the user could adjust accordingly to visualize their desired results. The results from the query are used if the user decides download the data from the website. In order to download data, the user had to use a single button. This button is associated with a geoprocessing service that selects the desired results and creates a zipped up shapefile that is sent to the user's temporary folder on their computer.

There was also a final functional requirement for the mobile device: position updates had to be required automatically for the user. This was completed by using an Android function that would repeat a particular set of tasks every given amount of time. The repeated tasks recorded a new observation in the local database, submitted the observation to the geodatabase using feature services, and rendered the new position update on the map.

\subsubsection{Non-Functional Requirements}

The technical requirements are the backbone of the non-functional requirements as they are focused on the technologies required to build the system. Web services were created from a geodatabase and published from within ArcGIS Desktop 10.0 to ArcGIS Server 10.0. These services were made available to users through the mobile and web applications.

The web application was composed of graphical user interfaces and application navigation. It was hosted using Internet Information Services (IIS) web server and required a universal resource locator (URL) for access. The web application must be accessed using Google Chrome or Mozilla Firefox. The application was developed using Notepad and Aptana environment and utilized Esri's ArcGIS Application Programming Interface (API) for JavaScript.

The mobile application was also composed of graphical user interfaces and application navigation. It was developed for the Android phone with an operating system of 2.3.3 or lower in the Eclipse Integrated Development Environment (IDE). The mobile application utilizes the Android Software Development Kit (SDK) and Esri's ArcGIS Runtime SDK for Android.

In order to fully understand both the operational and transitional requirements, it is important to note that the system was built as a prototype, or proof of concept, the client 
intended to use to get funding for development of a more permanent system. Given this, the prototype system was intentionally designed to have very few operational requirements, and this was done at the expense of security. This means that users were to edit the default data set. There was no versioning or automatic archiving of the data. It is the client's responsible to periodically download and archive the dataset. The client will also be responsible for the annual/semi-annual monetary requirements of maintaining a GIS server, web server, and website.

The system was developed for the general public, so there was no documentation or training made available to the client for using the mobile and web applications. The only other transitional requirement was the distribution of the mobile application. While this could be available to the public through Android's App Store, this was beyond the scope of the project. The client is responsible for personally downloading it onto the devices that will be using it.

\subsection{System Design}

The design of the system was guided by the system requirements described above. This section describes the system architecture design for both the mobile and web applications, along with constraints on the design. From this point forth, the system will be broken into two categories: server side and client side (Figure 3-1). This is done to clarify the difference between activity on the user side of the solution and the server side, where the system resides. The client side of the application sends requests to the server side, which then sends responses back to the client side. The server side is composed of the web server, ArcGIS Server, the application, and a geodatabase. The client side is split between the web component and the mobile component.

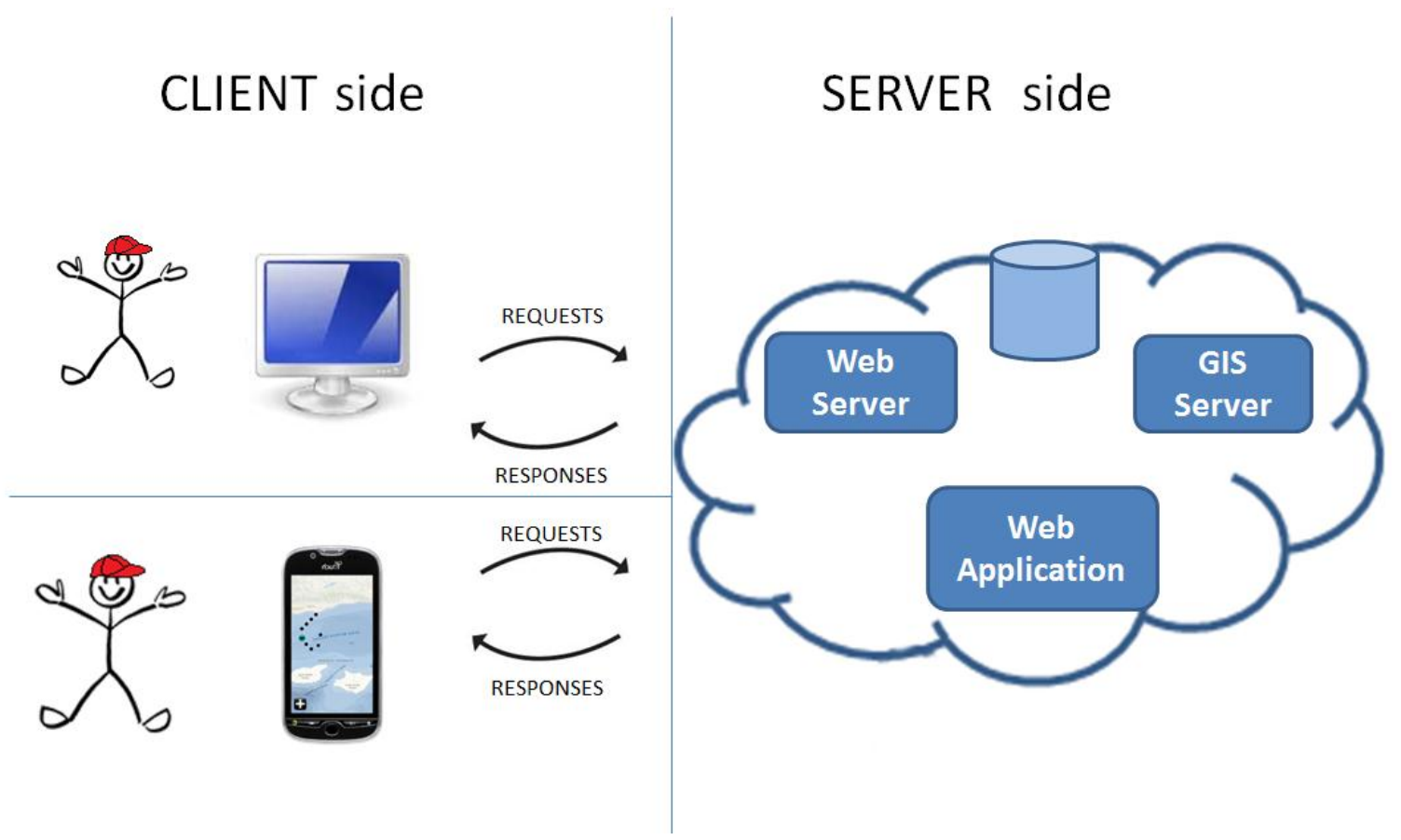

Figure 3-1: Overall system design 


\subsubsection{Web Application}

The web application was designed as a shell for future development of the application. It sits on the client side and consists of a user interface, along with supporting JavaScript functions. The JavaScript functions are responsible for the dynamic capacity of the web application and were developed using the DOJO JavaScript framework and the ArcGIS API for JavaScript. Both the JavaScript framework and the API were consumed by the application through services.

The user interface includes a Home, Map, My Observations, and a Learn tab (diagrammed in Figure 3-2 above). The Home and Learn tab were not developed during the scope of this project. The Map tab was designed for use by visitors that didn't want to log into the system. The map tab was designed to allow the user to visualize observations from a map service on top of Esri's Ocean basemap and query them by date and species type. Upon clicking on a particular observation, the application was designed to display the attributes and photo of the selected observation in a popup window. The Map's JavaScript methods are responsible for ensuring the queries are sent to the server and that observations are rendered on the map.

The My Observations tab requires users to log in before access. Just like the Map tab, this one was designed to also allow users to visualize and query the data, the difference being that the users are provided with additional query options.

The advantage of the My Observations tab is that users can download data after querying the database and after visually confirming the selection criteria they wanted. The My Observations tab was designed to allow users to submit new observations. This is completed using a feature service. Just like the Map tab, the dynamic functionality and server communication are handled by JavaScript methods in the My Observations tab.

There were several design constraints to take into consideration of the system design. The web application was designed to only work with a modern browser, such as Firefox or Chrome. Modern web browsers support a combination of standards, while earlier browsers only support very simple HTML standards. Additionally, the web application was designed to work with the ArcGIS API for JavaScript. This API was chosen because of its flexibility and popularity in the web programming field. It also is the only client-side web API that doesn't require a plugin for the browser.

\subsubsection{Mobile Application}

The mobile application has both user interface and supporting components. There are three components to the user interface design of the mobile application: the Home activity, the main activity called the Survey Map, and the Observation Activity. The supporting components on the mobile application's client side were the Local Database and the Events Database Manager.

The Home activity was responsible only for starting a trip and allowing users to decide which of their email accounts they wanted to associate their survey log with through a login window. The Survey Map was responsible for allowing users to visualize their trip. The activity also had a method for automatically recording and displaying the user's position every five minutes. In addition to recording the position update, the Survey Map added a new position marker to the map. Finally, the Survey Map was responsible for adding observations stored locally to the map. The Observation interface 
provides the user with a form to complete with a series of dialog boxes with necessary information.

There were multiple design constraints relevant to the client side of the mobile application. The first was the choice between a native and web application. The difference between these is discussed in more detail in Chapter Two. A native application was chosen for the system because one of the functional requirements was storing the data locally on the device. Having this workflow in place reduced future development burden of the application.

The second design consideration for the mobile device was the platform for which it would be developed natively. The Android platform was chosen because of Java's popularity and extensive documentation. It was also chosen because it is the operating system on more mobile devices than the completing Apple iOs platform. The application was developed for the Gingerbread operating system. The choices for the mobile platform and operating system were discussed in Chapter Two.

\subsection{Project Plan}

The project plan and implementation was similar to spiral and agile models for software development (Figure 3-2). After a requirements analysis, three separate phases were initiated: the geodatabase, the web application, and the mobile application. Each phase had the following steps: design, development, test, and discuss.

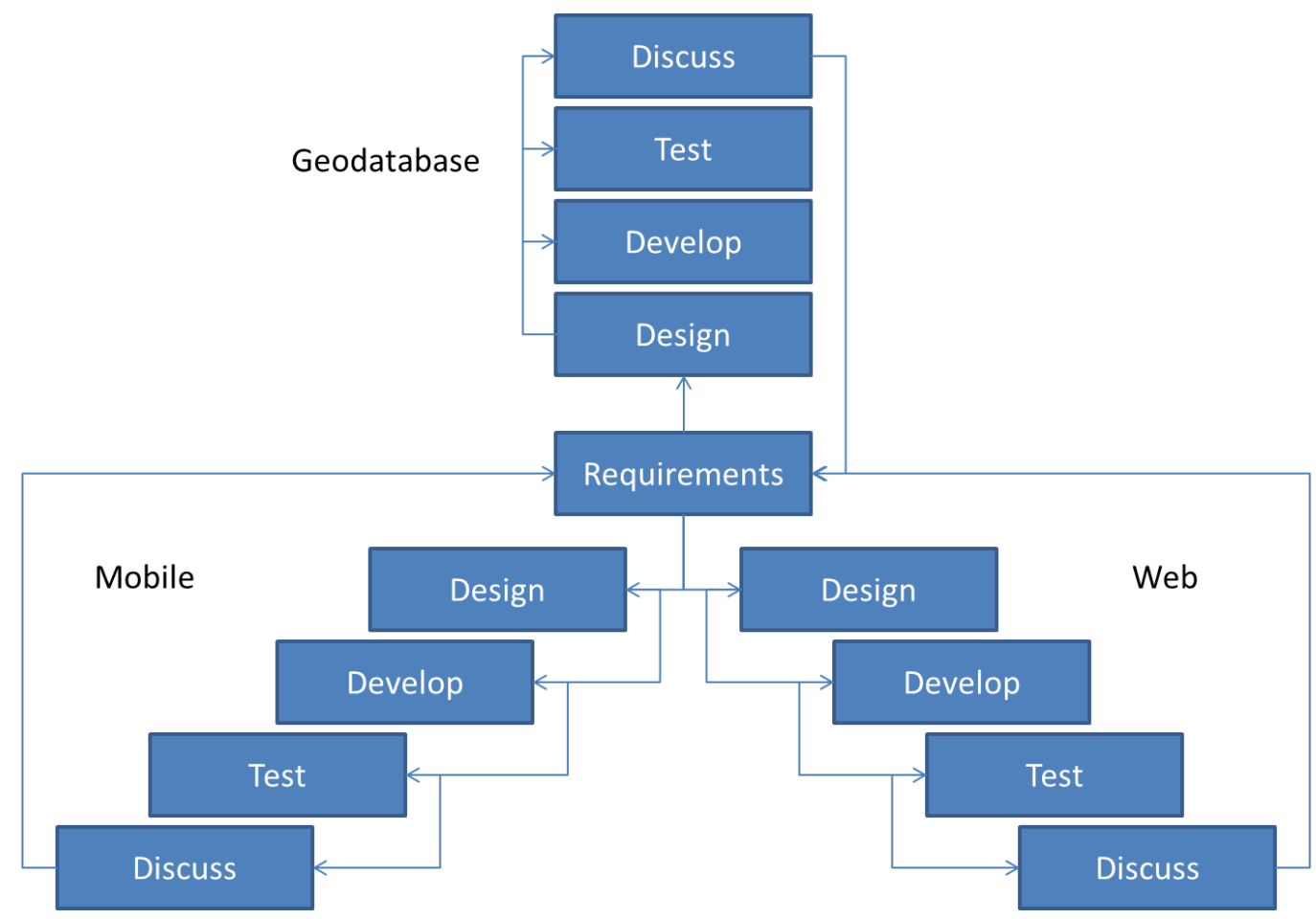

\section{Figure 3-2: A model of the project plan}

During the design task for the geodatabase, the client's functional and non-functional requirements were incorporated into a logical model. During every successive design task for both the web and the mobile applications, a mockup of the user interface was 
created and approved by the client. During the design and development stage for the web and mobile applications, the developer determined the appropriate functions that would be used in development through intensive research and training on topics including: the ArcGIS API for JavaScript, the Android Architecture, the Eclipse Integrated Development Environment, the Android SDK, and the ArcGIS Runtime SDK for Android.

During the development phase of the geodatabase, a logical model was developed. A logical model was created for both the client side (mobile applications) and the server side (geodatabase) of the system. Additonally, map and feature services were created. The development of the web and mobile applications were dependent on the completion of the geodatabase development.

Testing of the geodatabase and the client side applications were performed simultaneously. This was done because the web and mobile applications consumed services created from the geodatabase. In addition to the developer, the client was also asked to test the applications. After passing these tasks, a prototype was complete for each phase. During this time, a dialog was exchanged between the developer and the client. The client provided feedback on both the user interface and the functionality of the applications. Planning for the next prototype was then completed.

During the project development life cycle, some modifications were made. Testing the mobile application in the field gave insight, resulting significant modifications. For example, the original project plan was for complete offline editing on a mobile device. Field testing demonstrated that there was full connectivity in a region that could be used as the study area. Because of this, the mobile application no longer became a complete offline application. The application was designed to consume online basemaps rather than cached maps stored locally on the phone. However, the developer chose to continue storing the data locally on the device. This way, the only demand for mobile connectivity was when the user wanted to sync observations.

Additionally, the original plan included user authentication upon login and had the possibility of different permissions for different user types. Specifically, user authentication was planned to be done using openID (Google accounts), so all users must have a Google email account. However, because of the requirements of the server, this portion of the solution will not be implemented due to University regulations. Therefore, user verification was dropped from the implementation plan. Varied permissions were not incorporated into the applications, but their capability was demonstrated within the geodatabase.

\subsection{Summary}

The system requirements analysis process resulted in a system design that met the needs of the client. This chapter revisited the problem statement, discussed the requirements analysis and found that there were nine functional requirements and 13 non-functional requirements. In addition to the requirements analysis, the system design was also presented. This started with an overall description of the system design and continued with the design of the two client-side applications. Finally, the chapter concluded with a discussion of the project plan. The plan consisted of five major tasks and was adjusted throughout its implementation. With an understanding of the required functionality and system design, the database was ready to be developed. 



\section{Chapter 4 - Database Design}

The purpose of this chapter is to describe the data models used in the project solution. Section 4.1 describes the conceptual model, which was used to determine the classes and attributes needed to store the data required for the project. Section, 4.2 describes how the conceptual model was altered to balance data duplication and performance optimization in the logical model. Section 4.3 describes the data sources, and Section 4.4 describes the methods performed on the data before they were ready for use in development of the web and mobile applications.

\subsection{Conceptual Data Model}

The conceptual database model was developed through conversations with the client and the field data collection sheet (described further in 4.3). The conceptual model helped define the necessary database classes needed to solve the client's problem (Figure 4-1) These are the primary classes (shown in italics) that needed to be defined: Observer, Survey, and an Event. The relationship between the Observer and the Survey is that an Observer records surveys. E+9*-ach Survey contains one of more events. The Event table holds two different types of events: position updates and observations. All events must contain time and location. An event of the Observation type contains additional information. An Observation event may contain evidence, such as a photo. The Evidence class is the evidence, such as a photo, associated with a particular event.

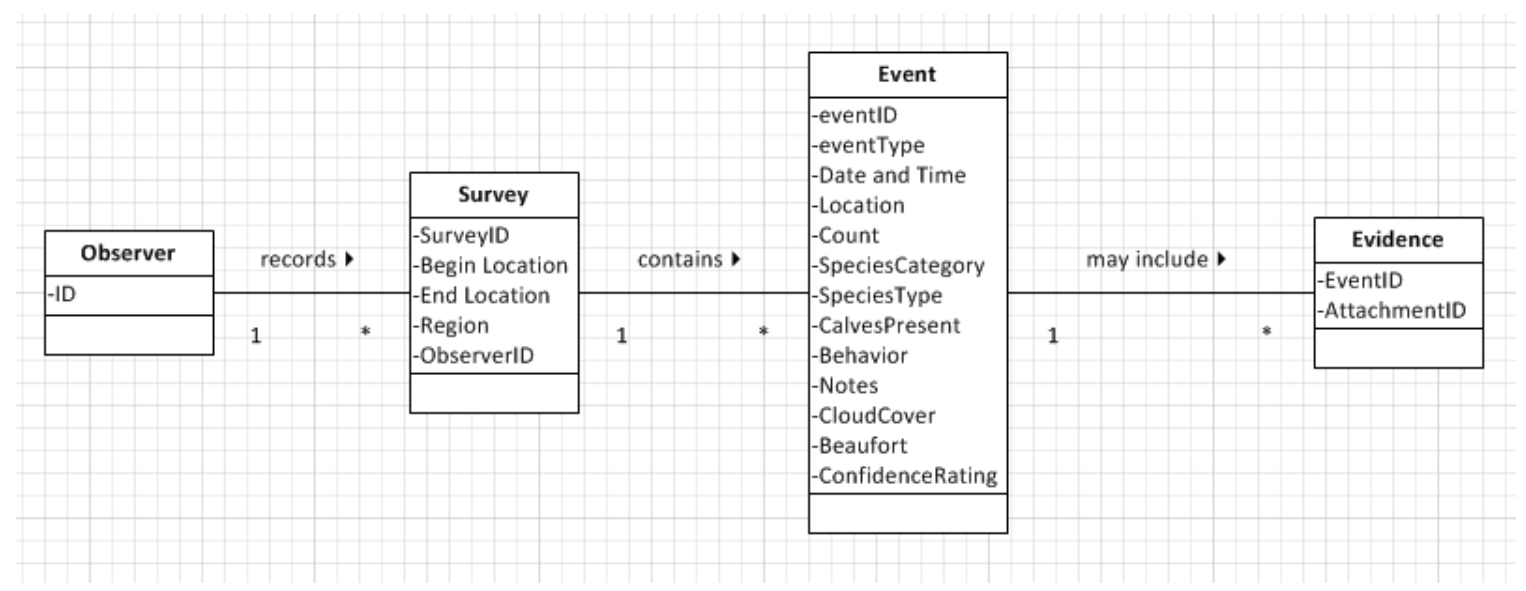

Figure 4-1: Conceptual Model

\subsection{Logical Data Model}

While the conceptual model is normalized and describes the client's needs abstractly, the logical model describes how the database schema was designed. The logical model design took into consideration the most appropriate way to store data for use in web and mobile-based applications. A diagram of the logical data model can be found in Figure 42 below. There are two extremes in data modeling: a completely normalized model and a flat file model. Flat-file databases have no explicit relations between tables, while normal databases have undergone a normalization process to eliminate data duplication 
and minimize the use of space. The advantage that flat-file databases have over normalized ones is that they perform much faster. This is desirable in databases used for web applications. The flat-file approach also reduces the learning curve for new administrators of the database.

Because of this, the Observer and Survey classes were consolidated into the Event table. This resulted in a geodatabase with only an Event table. It is important to note the consequences of using a flat-file database over a normalized one. There is significant data duplication in flat-file database and there are none in normalized ones. Additionally, the compartmentalized effect of normalized databases makes them easier to maintain and update. The use of domains and subtypes were incorporated into the database design to reduce maintenance demands. A complete listing of these can be found in Appendix A.

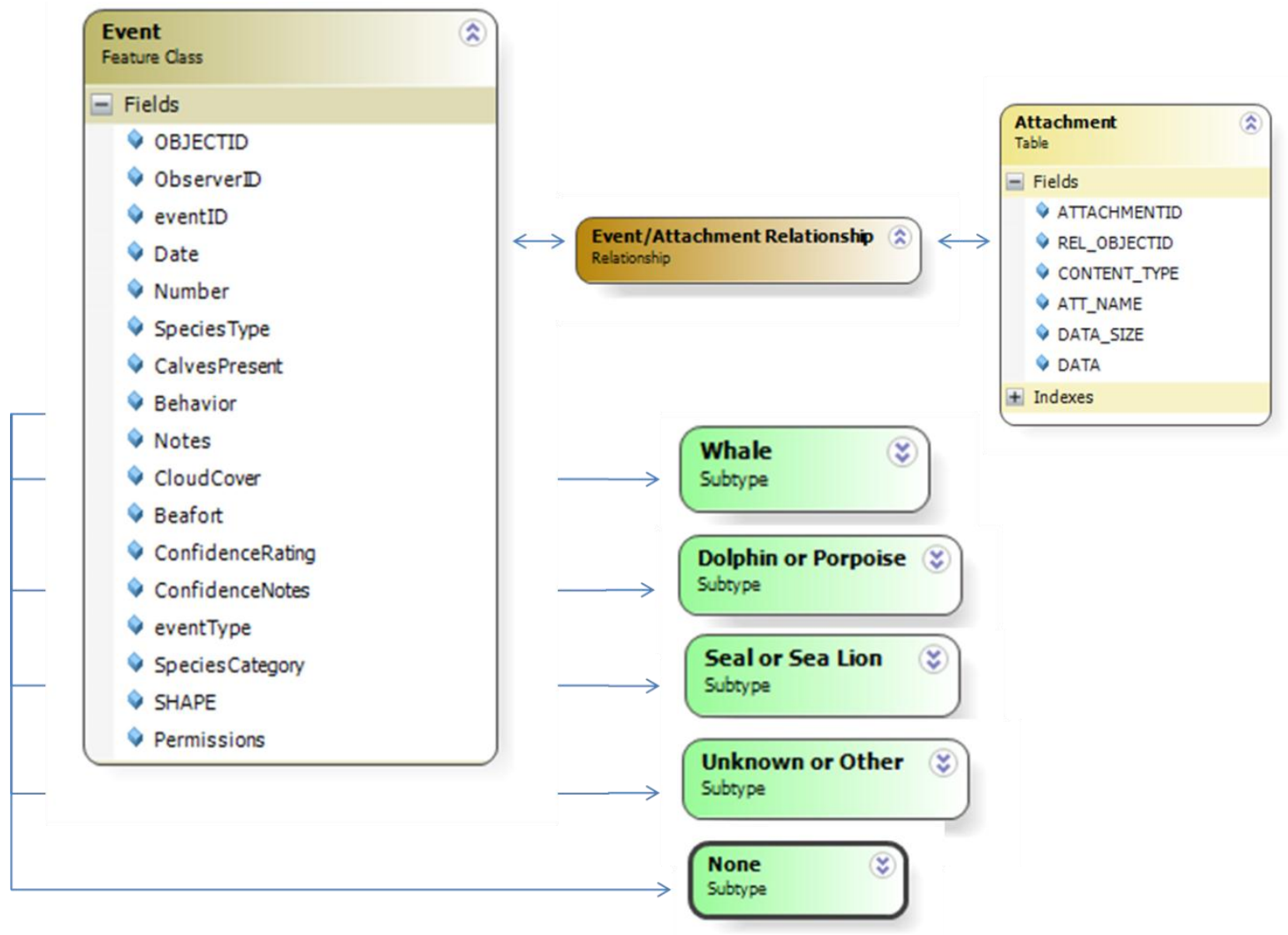

Figure 4-2: Server side logical model diagram

The data needed to be stored on both the local client mobile device and the server, so two different components to the logical model were created. The Event table was stored as a feature class in an ArcSDE geodatabase on the server side and it was stored as a table in a SQLLite database table on the mobile device. This is outlined in Figure 4-3 below. 

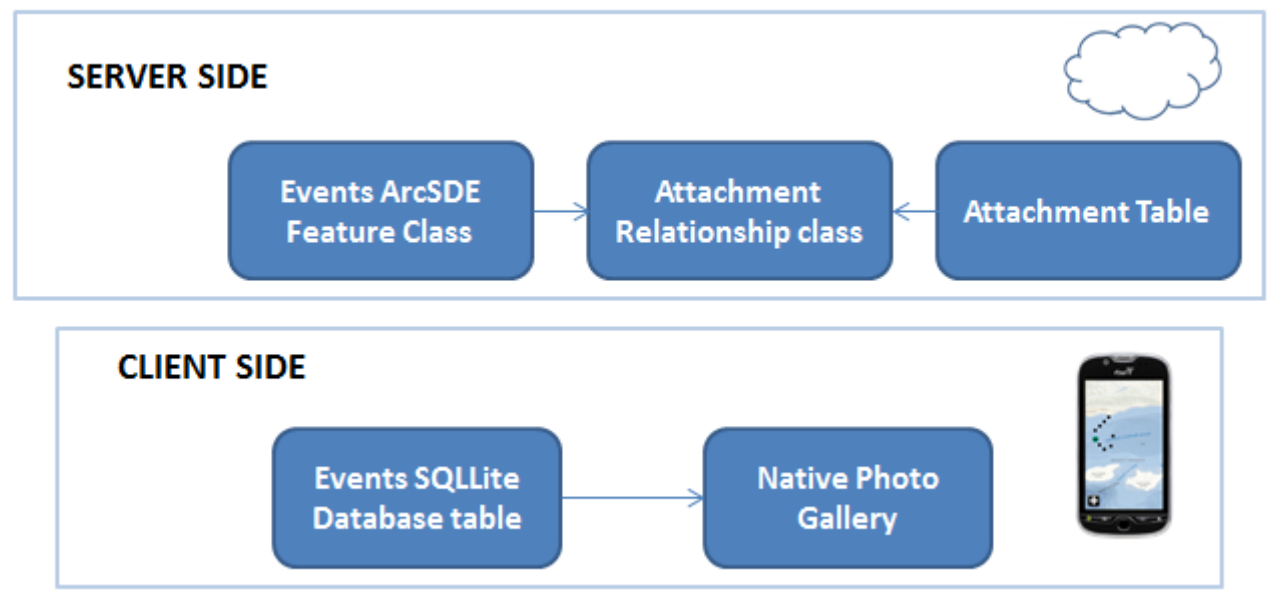

\section{Figure 4-3: Client side vs. server side database structure}

The primary difference between the way the data were designed to be stored in the logical model has to do with the way that evidence (photos) are associated with the events in the Event table. There are several methods for associating evidence, such as photos, with records in a table. These methods include storing a hyperlink as a text field, storing the evidence as a blob field, or storing the evidence in a separate attachment table. For the server side Events feature class, an attachment table was chosen as the method for storing the evidence because it is not dependent on path names internally or URLS on the web. Additionally, it keeps the evidence stored within the geodatabase without being queried every time the event record is queried. This increased the speed of queries, which is essential in web applications.

The photos were stored differently on the client side. The physical photos were stored in the phone's native gallery. Only a pathname was stored in the Events table. The only other difference between the server side and client side Event tables was that the client side table contained an additional field for tracking whether or not the records have been rendered on the map. 


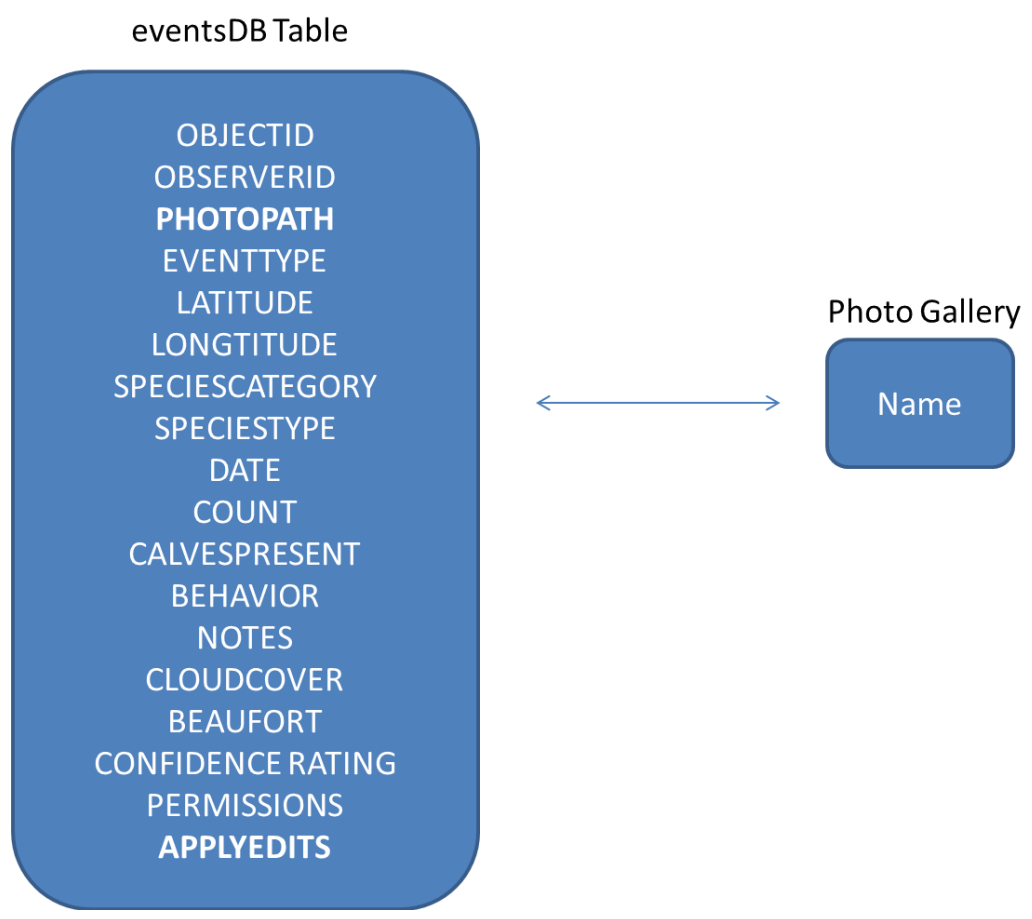

Figure 4-4: Client side logical model diagram

\subsection{Data Sources}

Recall that the project's mission was to build prototypes for collecting and managing marine mammal sightings. The development of the proof of concept prototypes didn't require much data for ensuring that the client's needs were met. Because of this, the data could have been synthesized.

Rather than creating a complete synthetic dataset, data were obtained from the Channel Islands National Marine Sanctuary (CINMS). CINMS has collected marine mammal sightings since 2003 and provided an Excel sheet with nearly 16,000 marine mammal sightings. Each sighting contained latitude, longitude, and a handful of other attributes, including: mammal category, type, date, vessel, location, count, and behavior.

In addition to the CINMS dataset, the data collection sheet used in the field was provided by the client. This data collection sheet is the form used by students and volunteers in the field (Figure 4-5). It was used to provide insight for the development of the conceptual and logical models. It was also used for development of domains for fields. 


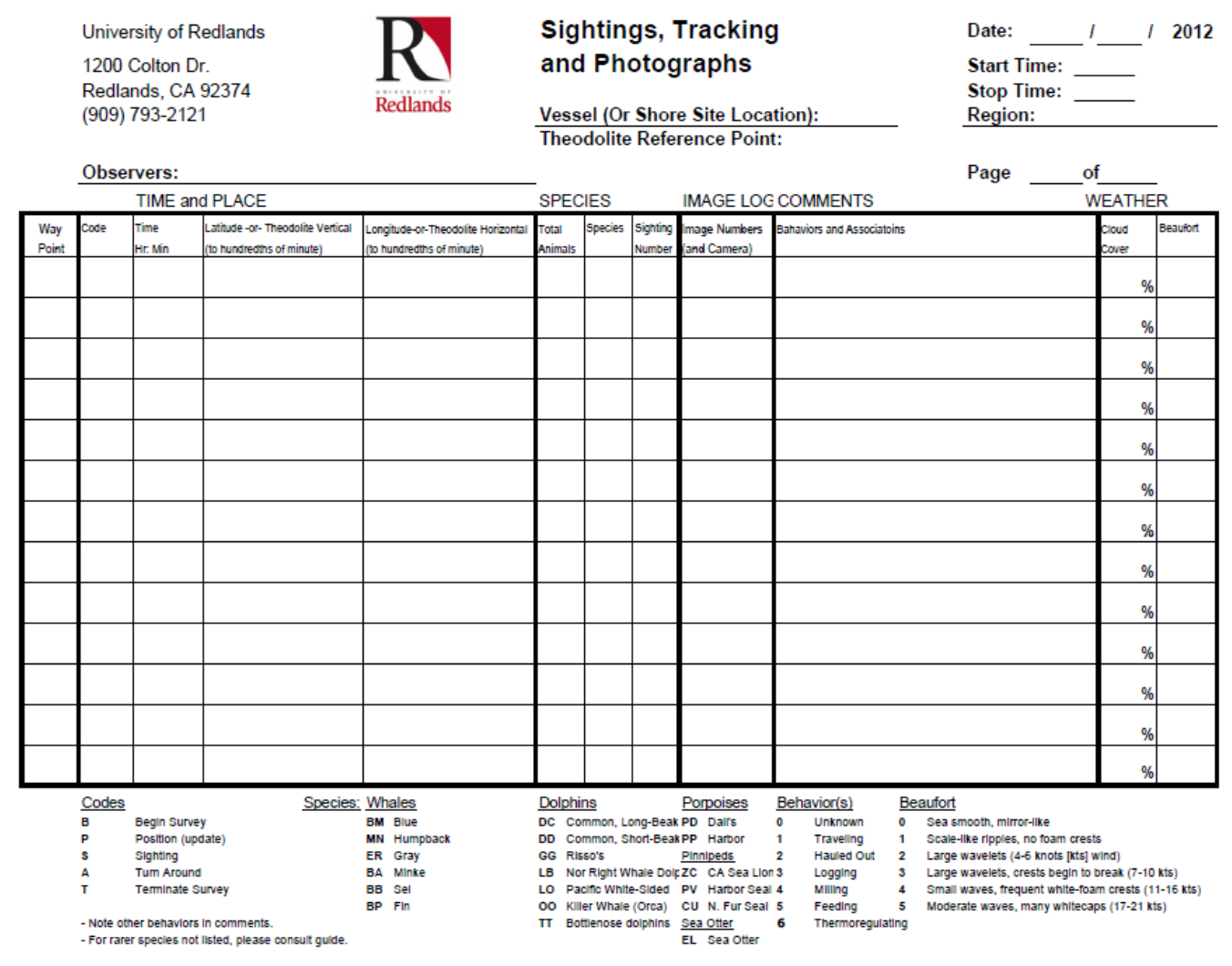

Figure 4-5: Data collection sheet used by client's students and volunteers

\subsection{Data Scrubbing and Loading}

Prior to loading the data provided by CINMS into the server side geodatabase, the geodatabase schema were developed. The schema included an Event feature class. The fields for the Event feature class included all of those listed in the logical data model in the preceding section. Domains for the feature class were created using the data collection sheet described in Section 4.3. Additionally, subtypes were created for four different species categories (Whale, Dolphin or Porpoise, Seal or Seal Lions, Unknown or Other). An additional category of "None" was created for position updates that had no observation data associated with them. The species categories were chosen for the subtypes to simplify the rendering of symbols and number of layers needed in the map service.

Prior to loading the CINMS dataset into the Event feature class, the longitude values were adjusted to a xy grid so that they would be stored in the correct hemisphere. Additionally, the CINMS was adjusted to include values for the coded values domains. Approximately 100 of the CINMS data points were imported into the geodatabase. Once in the geodatabase, several of the fields were either populated with synthetic values or left blank. The fields that were synthesized include: Observer ID, event type, date, event ID, calves present, cloud cover, beaufort, confidence rating, and notes.

The final step in prepping the data to be used in development was associating an attachment table with the events feature class via a relationship class, which was created. 
A photo was attached to each of the records in the Event feature class with an Observation subtype. The photos used were taken by Earthwatch volunteers during a whale watching trip.

\subsection{Summary}

This purpose of this chapter was to describe the database model and the data that were relevant to the project development. In the section on the conceptual model, the classes and their associated attributes that represent the problem proposed were described. The conceptual model consisted of four classes. The logical model was then discussed that was made during development of the solution for faster access of the data in both the client and server side environments. It consisted of two classes. The chapter also discussed the sample observation data provided by CINMS. It concluded with a discussion on the schema that was built and the scrubbing that was performed on that data prior to loading them into the geodatabase. With a well-designed database, and sample data ready for testing, the applications were ready to be built. 


\section{Chapter 5 - Implementation of the Web Application}

Two client side components were developed to meet the client's requests: a web application and a native mobile application. The mobile application was designed for users who are on personal or chartered whale watching boats and interested in tracking their trip and recording their observations instantly. On the other hand, the web application was designed for users who are either interested in visualizing the data as a visitor, or submitting single point observations and downloading the data for analysis as a logged-in user. This chapter describes the implementation of the web application. Section 5.1 discusses the web application's user interface. Section 5.2 discusses the functional components of the web application. The chapter concludes in Section 5.3 with a summary of the web application.

\subsection{Web Application User Interface}

The web application serves a variety of roles and was organized into four tabs: Home, Map, My Observations, and Learn (Figure 5-1). The Home and Learn tabs were completely undeveloped and acted as placeholders for future work, as requested by the client. The Map tab and the My Observations tab were divided into a content pane for a map object and accordion panes for the tools and forms available to the user. The accordion pane is a web control object that is displayed on the user interface. It appears as a container for holding web content. The map content pane is displayed to the user during the entire session, whereas accordion panes can be hidden and displayed as desired.

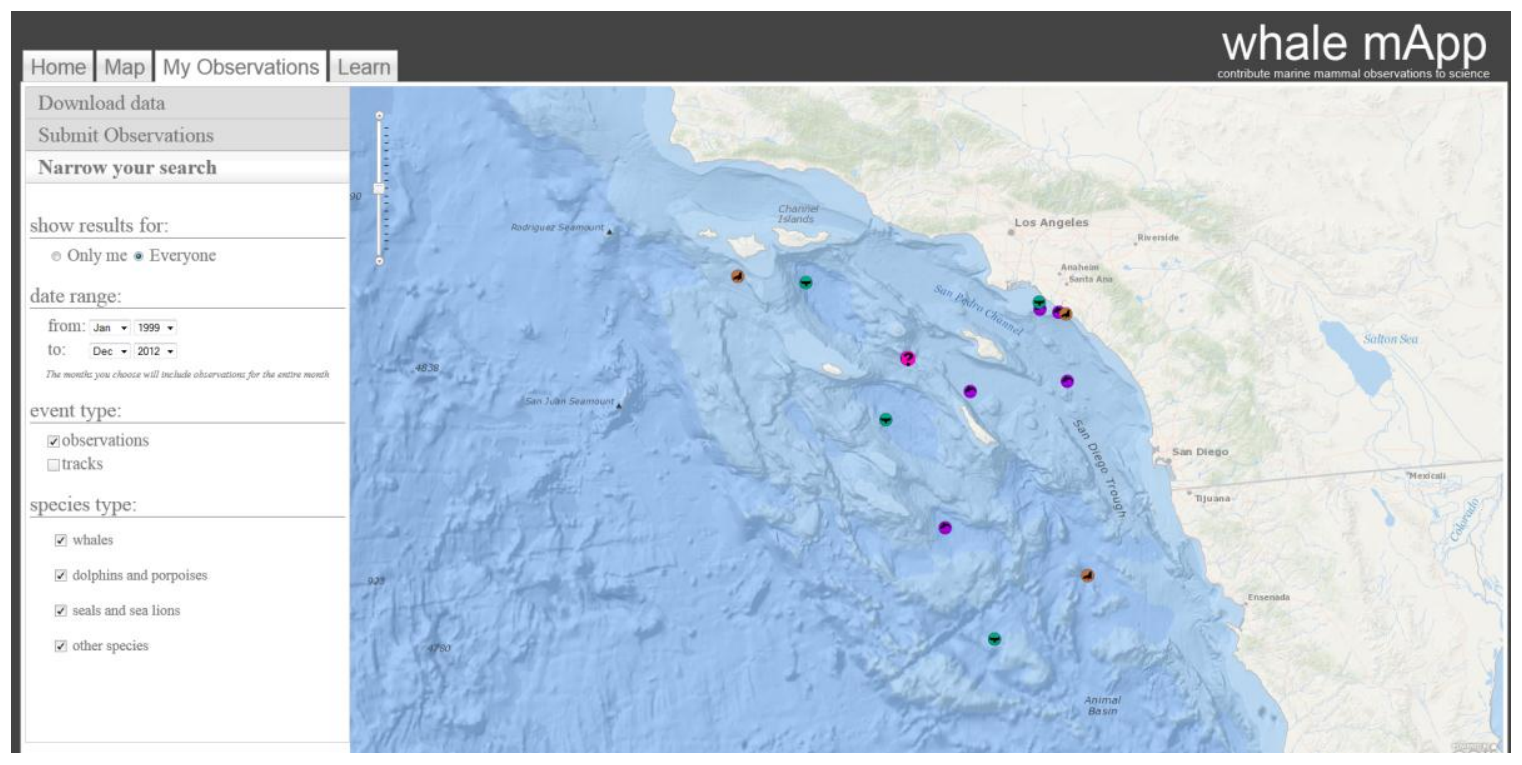

Figure 5-1: Functional components of the web application. 


\subsubsection{The Map tab}

The Map tab was designed for the users who don't wish to log into the system and are interested in visualizing observations, querying the database, and identifying details on selected events. It consists of several components (Figure 5-1), one of which is a map for visualization. The map is the central component of the tab. The tab includes an identify popup tool that allows the user to learn information about a particular observation. The tool triggers a popup when the user clicks on a symbol within the map. The identify popup tool was designed to be accessed only when a user clicks on an observation in the map object.

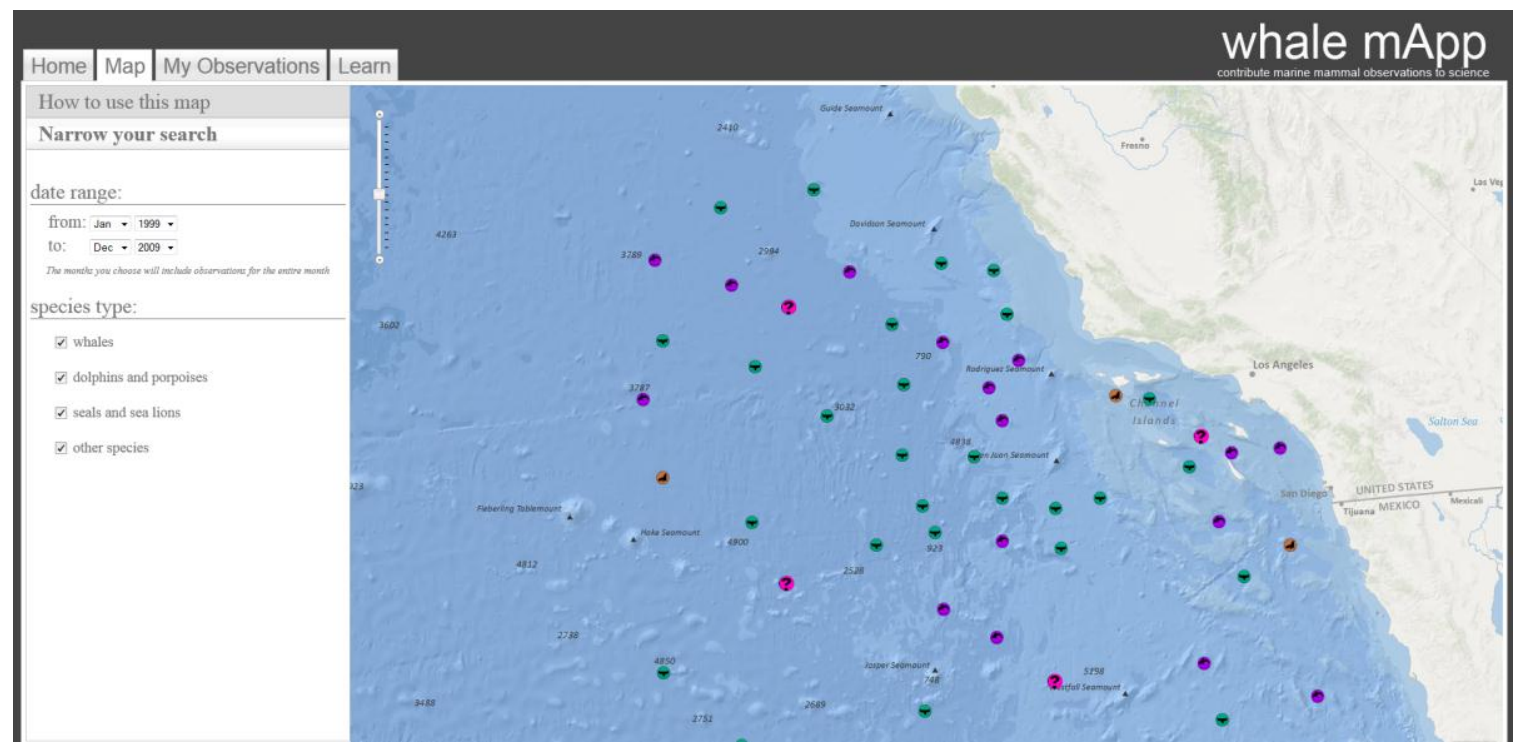

\section{Figure 5-2: Overview of the Map tab of the web application}

The user interface was also designed to include basic querying tools. Querying tools select the chosen records and only displays them to the user. These basic querying tools are made available to the user through a content pane on the left side of the tab. The date range query tool allows the user to query by a "to and from date" using drop down boxes for month and date. These date tools are illustrated in Figure 5-3.

$$
\begin{aligned}
& \text { date range: } \\
& \text { from: Jan } \\
& \text { to: Dec }-2012 \\
& \text { The months you choose will inchude observations for the entire month }
\end{aligned}
$$

\section{Figure 5-3: Date Query Tool}


The user can also query by species type. Species type options are made available to the user through checkboxes (Figure 5-4). This allows the user to view results of any combination of species at the same time.

\section{species type: \\ $\checkmark$ whales \\ $\checkmark$ dolphins and porpoises \\ seals and sea lions \\ other species}

\section{Figure 5-4: Species type query tool}

\subsubsection{The My Observations Tab}

The My Observations tab was designed for an active user interested in a more advanced interaction with the data (Figure 5-5). When the user accesses the tab, they are prompted to $\log$ in. Upon log into the system, the user has access to the same map, and identify popup tool as in the Map tab.

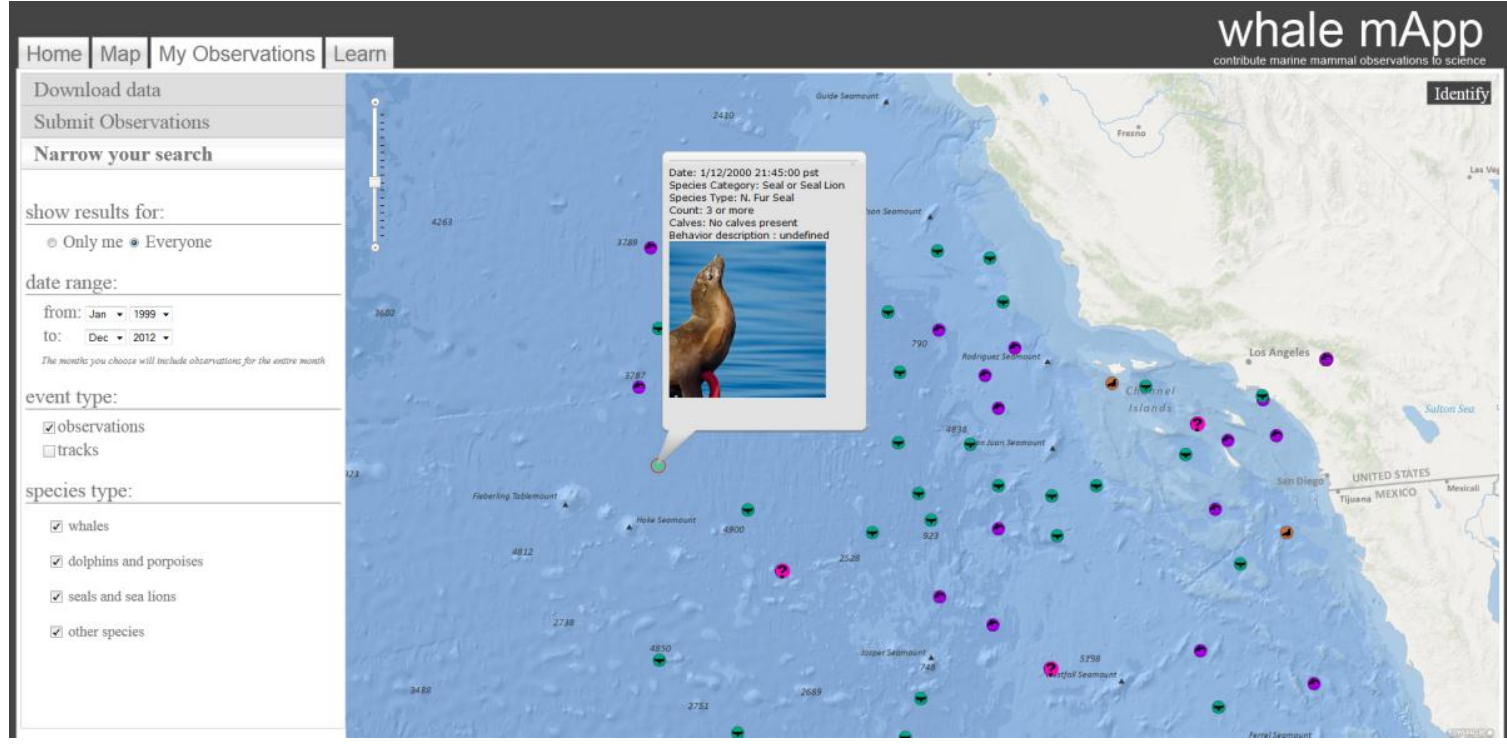

\section{Figure 5-5: My Observations tab}

This tab has more advanced query tools which are displayed within an accordion pane. The user can query by event type, allowing them to see both observations and tracks. This query tool is made available to the user as checkboxes, allowing them to view any combination of event types (Figure 5-6). 


\section{Figure 5-6: Event type query tool}

They can also choose to see only their observations or the observations of all users. These options are made available to the user through a radio button, and they can choose only one option or the other (Figure 5-7).

\section{show results for:}

\section{○ Only me ๑ Everyone}

\section{Figure 5-7: Show results query tool}

Additional accordion panes were designed for additional tools, one of which is the download data tool, which allows users to download data as a shapefile after they have queried the data with the desired attributes (Figure 5-8).

Download data
Found what you're looking for?
Click "Extract Data" to get a shapefile of
your selection
Extract Data

\section{Figure 5-8: Download data tool}

Also within an accordion pane, the user has access to a Submit Observations Form (Figure 5-9). This form contains all of the information required to submit a new observation, along with the button needed to submit it. Most of the form's components were made from Dojo form widgets, also known as Dijits (Dojo, 2011). 


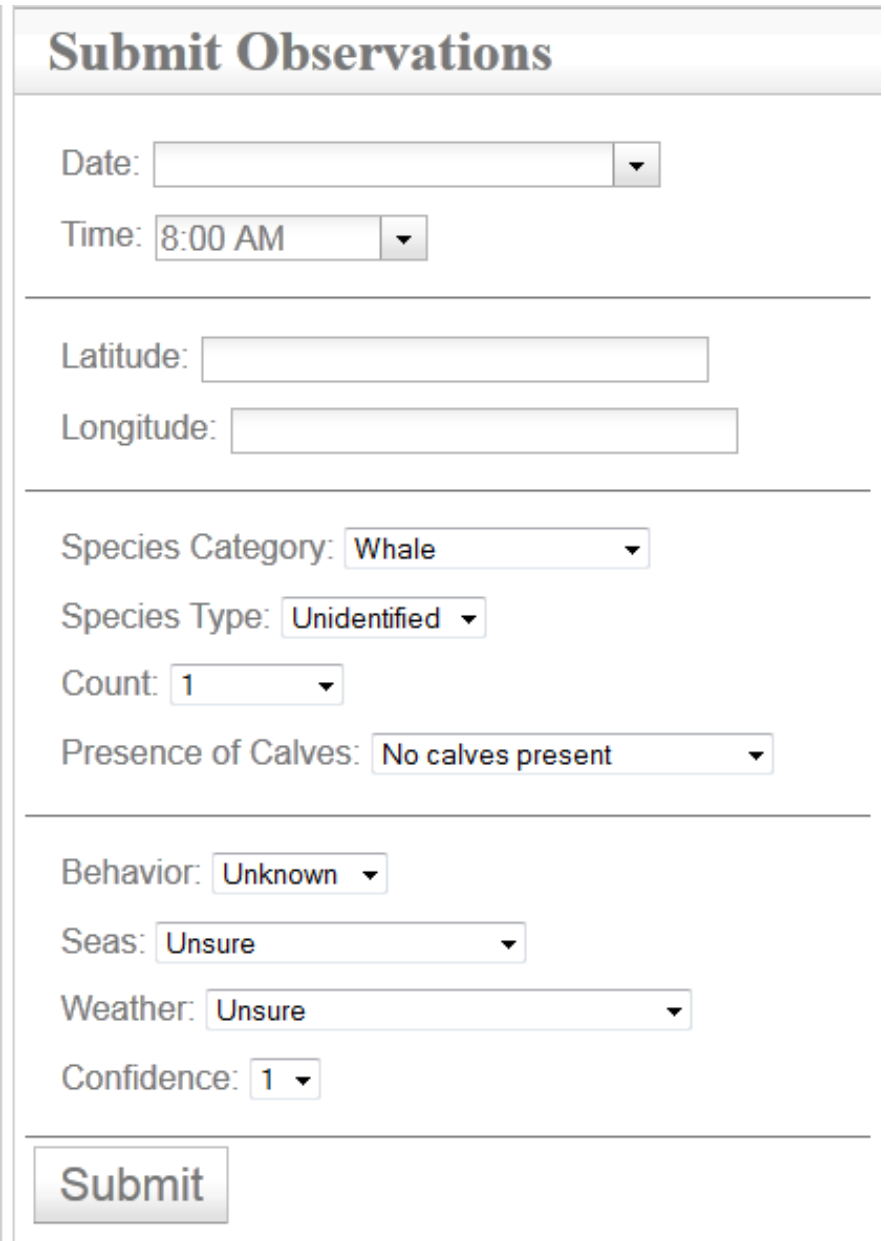

\section{Figure 5-9: Submit observations for in accordion pane}

The Submit Observations tool was designed to minimize the amount of error in data entry. This was done using drop-down lists and auto-fill for every option other than location (Figure 5-10). A NumberTextBox (a Dijit) was used to restrict the user to only entering numbers in the locational text boxes.

\begin{tabular}{|c|c|c|c|c|c|c|}
\hline \multicolumn{2}{|l|}{ ( ) } & \multicolumn{3}{|c|}{ October - } & & \multirow{2}{*}{$\begin{array}{l}\text { (1) } \\
\text { S }\end{array}$} \\
\hline $\mathrm{S}$ & $M$ & $\mathrm{~T}$ & W & $\mathrm{T}$ & $\mathrm{F}$ & \\
\hline 30 & 1 & 2 & 3 & 4 & 5 & 6 \\
\hline 7 & 8 & 9 & 10 & 11 & 12 & 13 \\
\hline 14 & 15 & 16 & 17 & 18 & 19 & 20 \\
\hline 21 & 22 & 23 & 24 & 25 & 26 & 27 \\
\hline 28 & 29 & 30 & 31 & 1 & 2 & 3 \\
\hline 4 & 5 & 6 & 7 & 8 & 9 & 10 \\
\hline & 201 & & 2012 & & 13 & \\
\hline
\end{tabular}

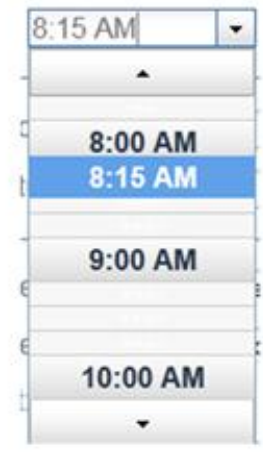

\begin{tabular}{|l|}
\hline Whale \\
Whale \\
Dolphin or Porpoise \\
Seal or Sea Lion \\
\hline
\end{tabular}

Figure 5-10: The use of auto-fill and drop-down use for the Submit Observations form 


\subsection{Functional Components}

The user interface components described above are dependent on several functional components. The purpose of this section is to describe each of the functional components in detail. The functional components and their corresponding classes and methods are illustrated in Table 5-1. Classes are shown in lower camel case (ExampleClassName) and methods are shown in lower camel case (exampleMethodName).

Table 5-1: Functional components and their corresponding methods

\begin{tabular}{|l|l|}
\hline Functional Component & Class or Method \\
\hline The Map & Map \\
\hline Identify Popup Tool & identify \\
\hline Query Tools & $\begin{array}{l}\text { dateChanged, speciesChanged, } \\
\text { changeLayerDefinition, } \\
\text { eventTypeChanged, } \\
\text { showResultsFor }\end{array}$ \\
\hline Download Data Tool & downloadData \\
\hline
\end{tabular}

\subsubsection{The Map}

As mentioned, the map appears in both the Map and My Observations tabs. The map's content is dynamic, depending on the extent, map layers, and query functions. The map extent was set to (in meters):

- $-13,252,801.136300$ (minimum $x$ )

- $4,388,898.711300$ (minimum y)

- $\quad-1,4091,002.8127$ (maximum $\mathrm{x}$ )

- 3,508,363.61690 (maximum y).

The layers contained within the map component are outlined in Table 5-2 below. The map, feature, and geoprocessing services that the map layers were composed of were published through ArcGIS Server from a map document within ArcGIS Desktop.

Table 5-2: Web application layers

\begin{tabular}{|l|l|l|l|}
\hline Layer Name & Type & Usage & Mode \\
\hline $\begin{array}{l}\text { Ocean } \\
\text { Basemap }\end{array}$ & Basemap & Basemap & NA \\
\hline Events & $\begin{array}{l}\text { Feature } \\
\text { Service }\end{array}$ & $\begin{array}{l}\text { Display of } \\
\text { all } \\
\text { observations }\end{array}$ & Selection \\
\hline Attachments & $\begin{array}{l}\text { Map } \\
\text { Service }\end{array}$ & $\begin{array}{l}\text { Table of } \\
\text { image } \\
\text { attachments }\end{array}$ & NA \\
\hline ExtractEvents & $\begin{array}{l}\text { GP } \\
\text { Service }\end{array}$ & GP tool & NA \\
\hline
\end{tabular}




\subsubsection{Identify Popup Tool}

When the user clicks on an observation on the map document, the identify method is called. A query is sent to the server for any feature within the spatial extent of the envelope. If something is returned, a new selection symbol is defined for the events feature layer. The layer definition is reset (using the Map object's setLayerDefintion and setSelectionSymbol methods) with the new selection and selection symbol. This replaces the species category symbol with the unique selection symbol (Figure 5-11). This unique selection symbol has a green fill and red outline.

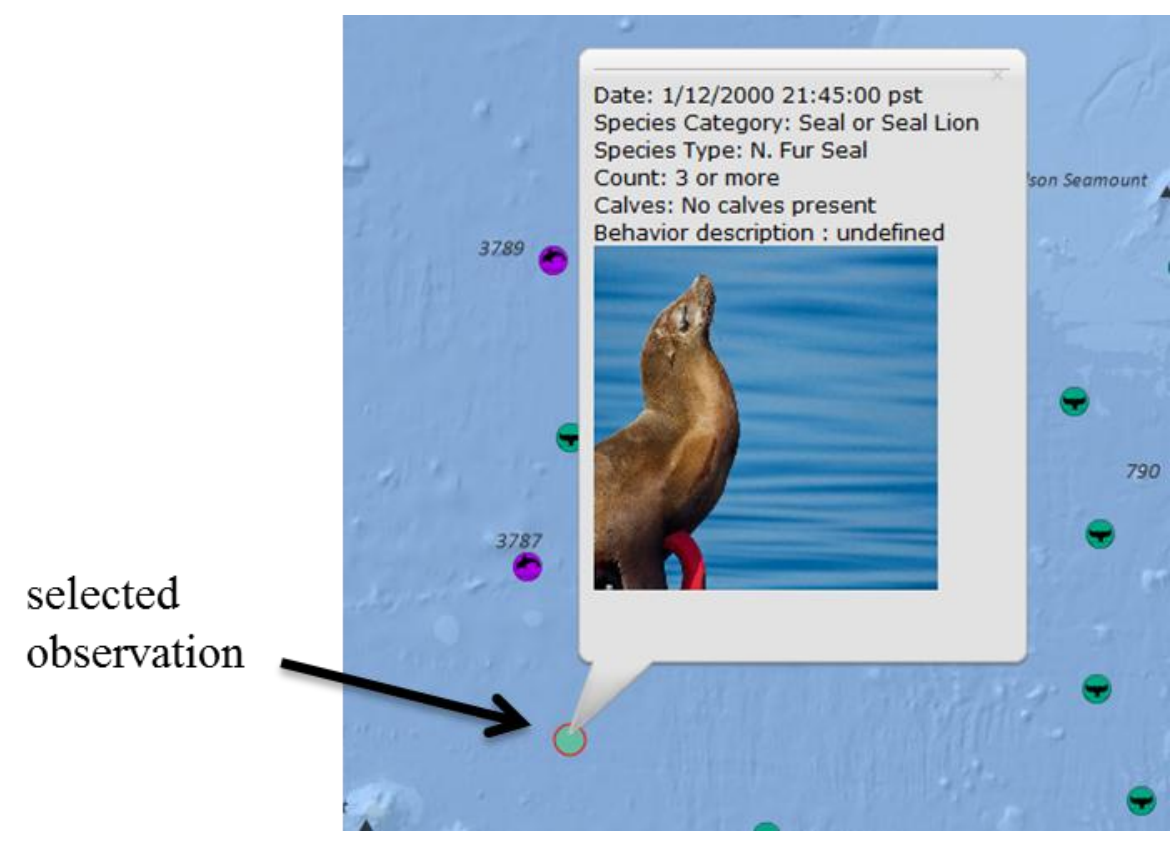

Figure 5-11: Symbol selection and popup window.

After the observation is assigned a new observation symbol, a second query task is defined and executed which searches the attachment table for a picture corresponding to the selected observation. If an image exists in the attachment table, the content of the map's infoWindow is updated, the window is resized, and the infoWindow is displayed.

\subsubsection{Query Tools}

The methods that perform the query are triggered by listeners on each of the query tool options. A listener is an object that performs an action when the user interacts with its corresponding part of the user interface. Each listener calls a respective function that builds a string to query its corresponding attribute in the feature layer. For example, when the user changes the date in the query tools, the dateChanged method is called. Afterwards, the changeLayerDefinition is called which pieces together the appropriate string consisting of each query tool's current value. The function then applied the query string to the events feature layer's layer definition. When applied, a request is made to the server and the server responds with the appropriate information for 
the features that need to be added to the map. The events feature layer is then reset using the Map object's setLayerDefintion method.

\subsubsection{Download Data Tool}

The downloadData function is triggered by a listener on the Extract Data button that appears in the Download Data accordion pane. The function uses the current download data query string that is built through the query tools. If the user has not narrowed their search with the use of the query tools, a default query string is used.

Upon activation, the application sends a request to a geoprocessing service with the required parameters. The geoprocessing service was published from a tool made with the ArcGIS Model Builder (Figure 5.12). The tool is responsible for selecting records from a feature class, extracting the data into shapefile format, and delivering the shapefile in a zipped folder to the user as a downloadable file.
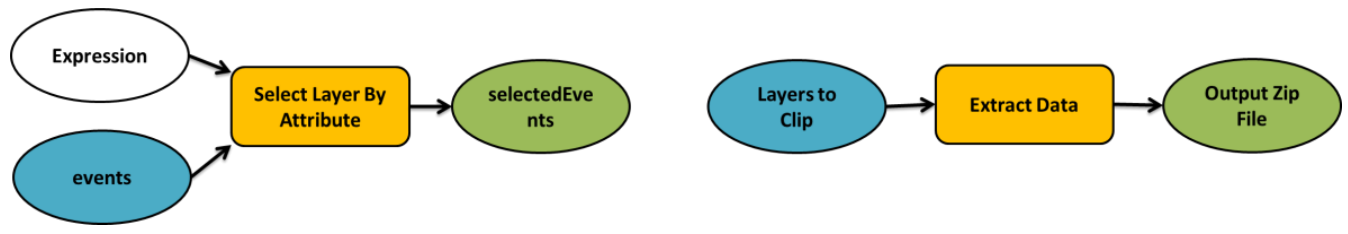

\section{Figure 5-12: Workflow of Extract events tool}

\subsubsection{Submit Observations Form}

When the user completes the Submit Observations form and pushes the Submit button from the user interface, a function that is responsible for submitting the observation event data is called. Within the function, a graphic object is created with the geometry specified in the latitude and longitude NumberTextBoxes (a Dijit) and the attributes specified by each of the other options in the form. The user ID entered upon the user's visit to the site is also saved as an attribute of the observation. Once the graphic is created, it is sent to the server using the ArcGIS API for JavaScript function applyEdits function that can only be called on feature services.

\subsection{Summary}

The web application consists of two tabs, Map and My Observations, intended for two different types of users. The tabs are composed of a map, an identify popup tool, and query tools. The My Observations tab also includes a Submit Observation form and a Download Data tool. These tools compose the functional requirements of the application and drive the dynamic components of the website. 


\section{Chapter 6 - Implementation of the Mobile Application}

As stated, the mobile application is intended for users who are on the water and are interested in tracking their trips and submitting observations. The application was designed to allow users to visualize their trip and record survey events (tracks, observations) locally on the device in addition to syncing them with the server. This chapter discusses the implementation of the mobile application. Section 6.1 describes the User interface of each of the application's Views. Section 6.2 describes the functional components of each of the Views.

\subsection{Mobile Application's User Interface}

There are three Views associated with the mobile application: Home, Survey, and Observation. Each of these Views is associated with an Activity, which is essentially a custom class. From this point on, all future references to Activities will be in UpperCamelCasing and Views will be in lowerCamelCasing.xml followed by .xml. Upon launch of the application, the user is presented with the home. xml View (Figure 6-1). This View has the application's title, a settings button, and the "Start a Trip" button.

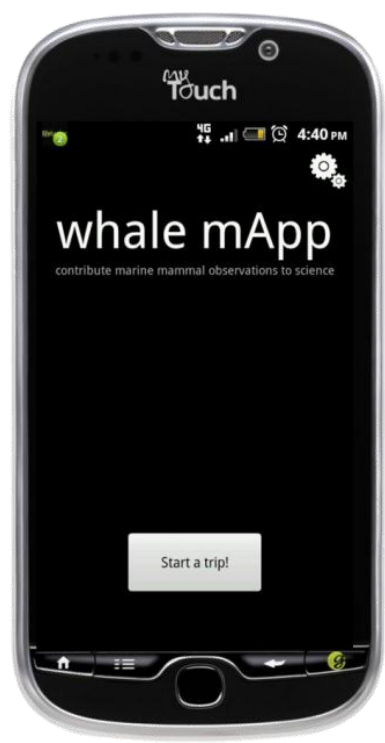

\section{Figure 6-1: Home View of the mobile application}

On selecting the settings menu, the user is allowed to only change the username that will be associated with the observations they submit. The list made available to the user to choose from is composed of "anonymous" along with each of the email accounts associated with their phone (Figure 6-2). 


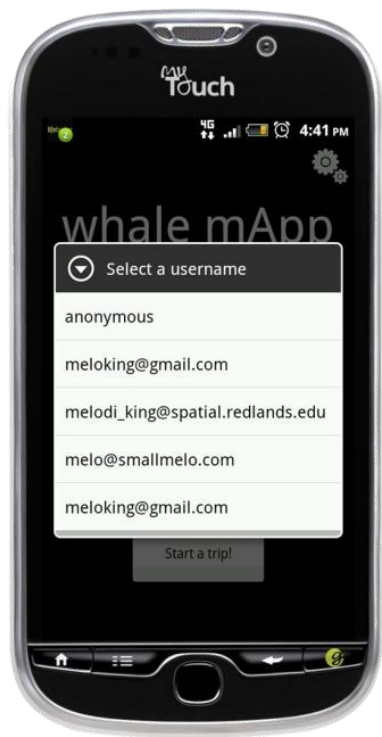

\section{Figure 6-2: Settings Menu}

The "Start a trip" button simply closes the home . xml View and brings the user to the Main Activity of the application: the SurveyMap. The surveyMap.xml view is composed of a map and a button that allows the user to record the observation (Figure 6-3). This View allows the user to visualize their trip with minimal effort. Their current location is represented by a blue dot. Every five minutes, the user's location is recorded as a position update and a new black dot appears on the screen. Each time a new observation is recorded, a symbol is added to the map to their marine mammal's category: whale, dolphin or porpoise, seal or sea lion, or unknown.

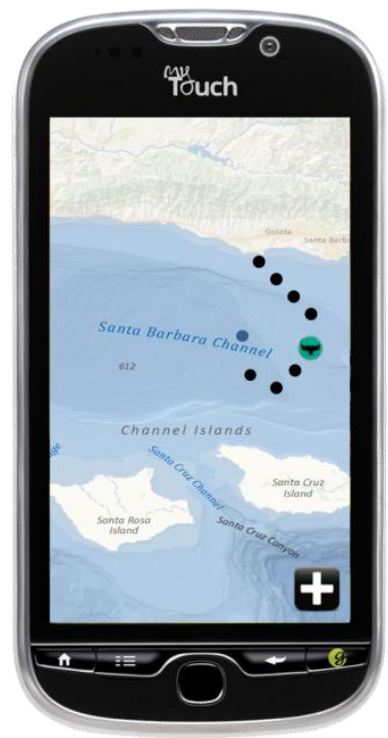

Figure 6-3: Survey Map View 
When the user selects the button to record a new observation, they are presented with a form to fill out (Figure 6-4) in the observation. xml View of the Observation Activity. The user must select a species category before any other information can be added to the form. After selecting a category, the user can scroll through the remaining details to add. The required information is bolded and the unrequired information isn't.

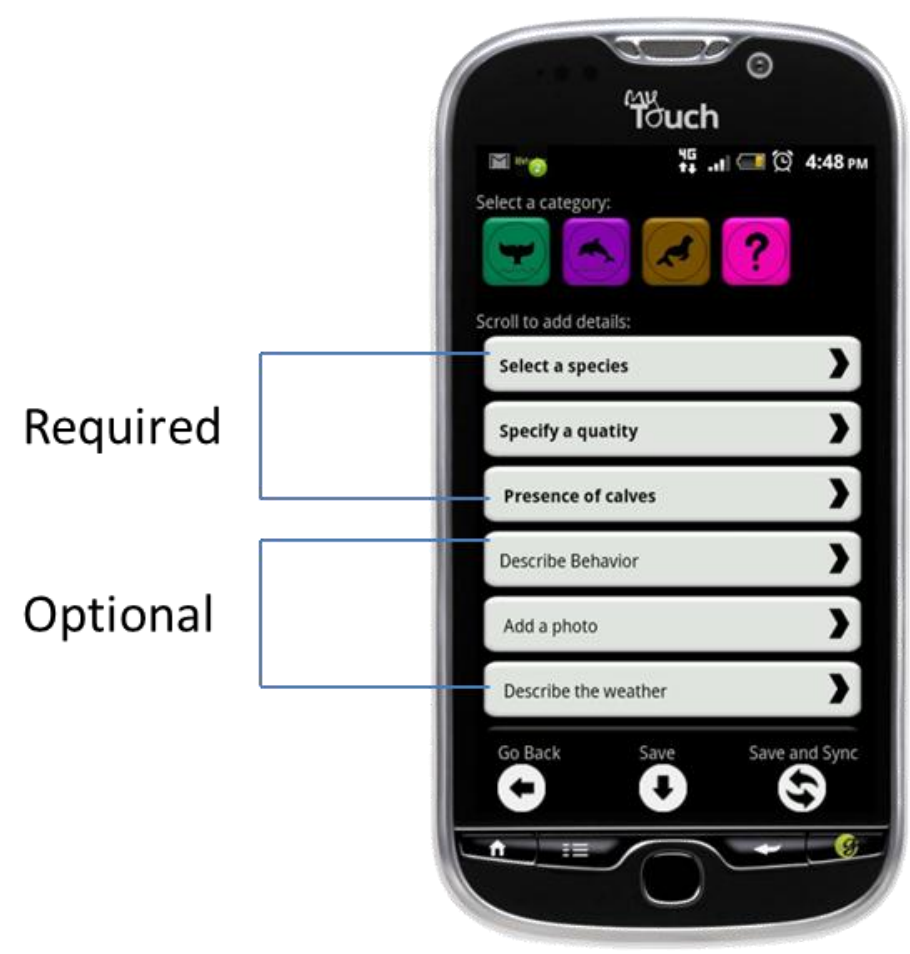

Figure 6-4: Observation View showing the required and optional components in the form

Each time the user selects one of the components, they are presented with a dialog box with options to choose from. Figure 6-5 illustrates each of the dialog boxes that are presented when the user selects the whale category. 


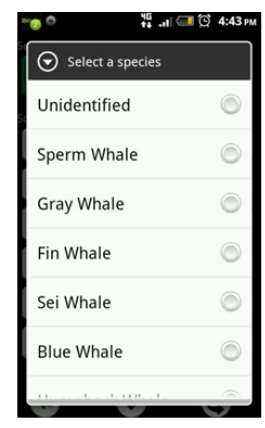

A) Select a whale species

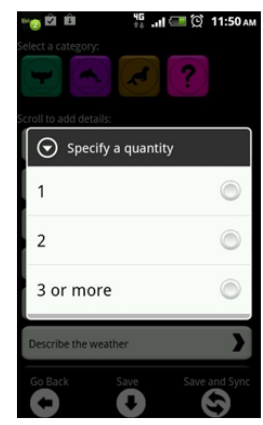

B) Select a quantity

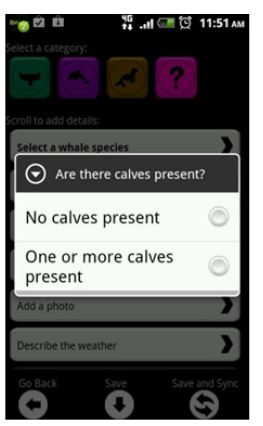

C) Presence of Calves

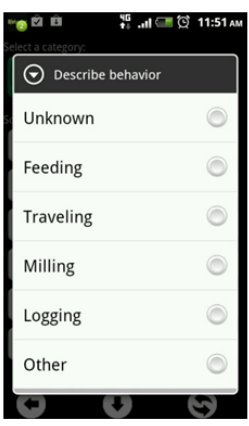

D) Describe Behavior

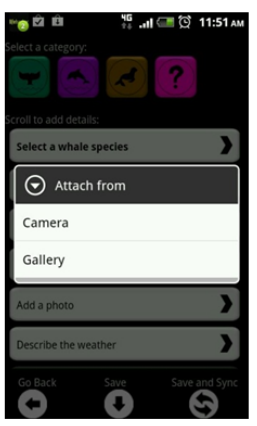

E) Add a photo

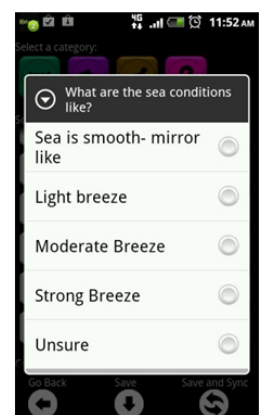

F) Describe the Beaufort

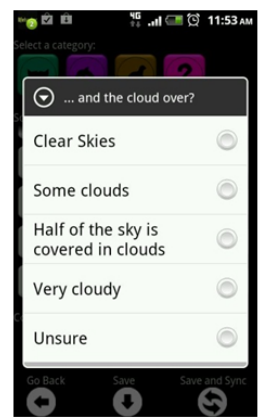

G) Describe the weather

Figure 6-5: Screenshots from the dialog boxes in the Observation form

At the bottom of the observation . xml View, the user has the option to "Go Back," "Save," and "Save and Sync." The "Go Back" closes the Observation Activity without saving. The "Save" button saves the observation locally on the phone, and the "Save and Sync" button saves the data locally on the phone in addition to syncing it with the server.

\subsection{Functional Components of the Activities}

This section describes the functional components of the mobile application by the Activity that they are associated with. Each of the Activitity's methods will be in lowerCamelCasing () followed by parenthesis and all objects appear in lowerCamelCasing.

Essential to understanding the functional components of the application is the Android Life Cycle (Activity, 2012). The life cycle describes the states and workflow of how applications are handled in devices running an Android operating system. Figure 66 illustrates the life cycle. Most of the functional components occur in the onCreate () function, but the onResume () and onPause () also play an important role in some cases. 


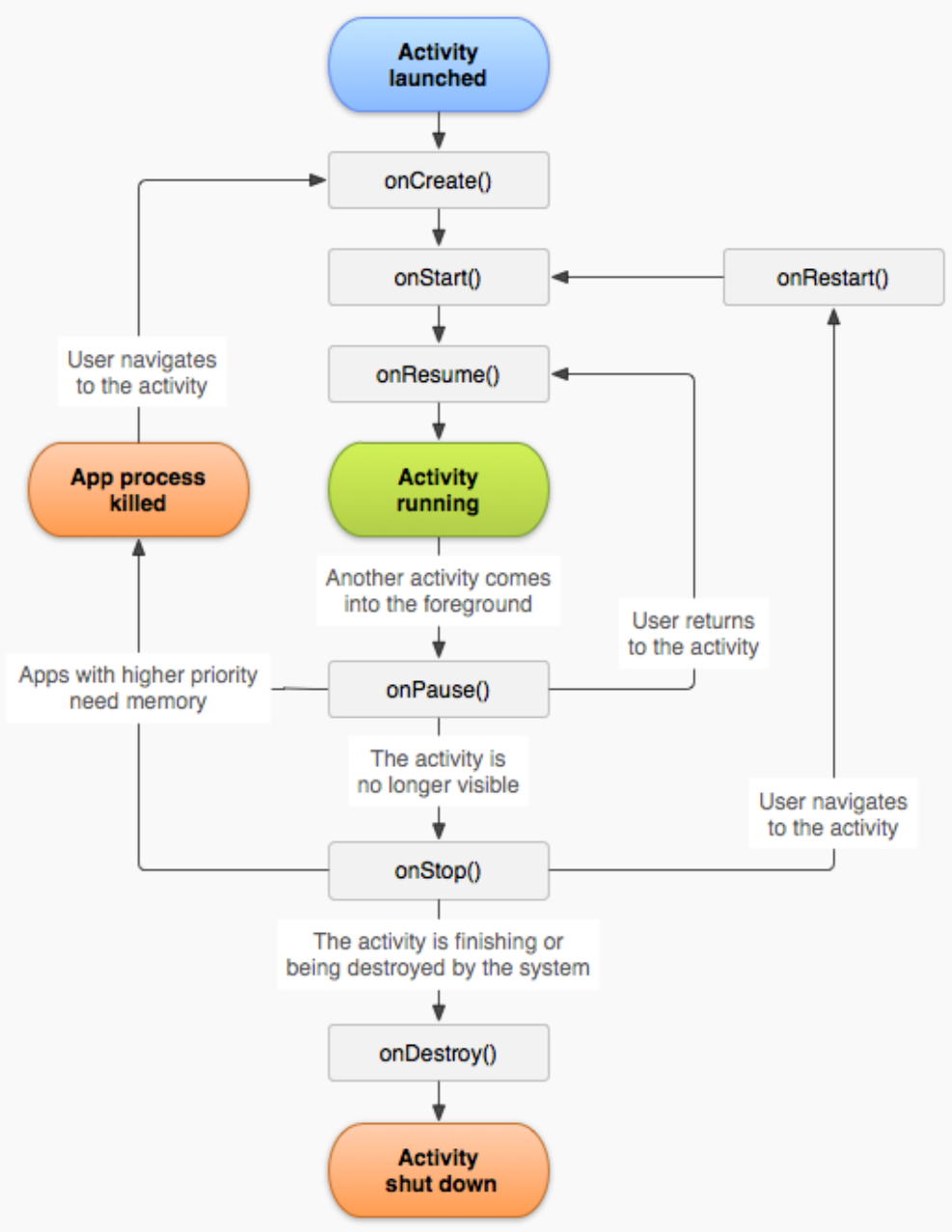

Figure 6-6: Android Life Cycle

Source: http://developer.android.com/reference/android/app/Activity.html

While the Home Activity is essential to the application, it contains no functional components. The two Views with functional components in their Activities are the surveyMap.xml and the observation. xml View. There is also a helper Activity with functional components that doesn't have a View: the EventsDatabaseManager. This Activity is responsible for handling the data stored locally on the device.

\subsubsection{Survey Map Activity}

The SurveyMap Activity has several functional components which include: the map object, positionUpdates () method and handler, onStatusChange Listener, and the updateEventsLayer ( ) Method (Figure 6-7). 


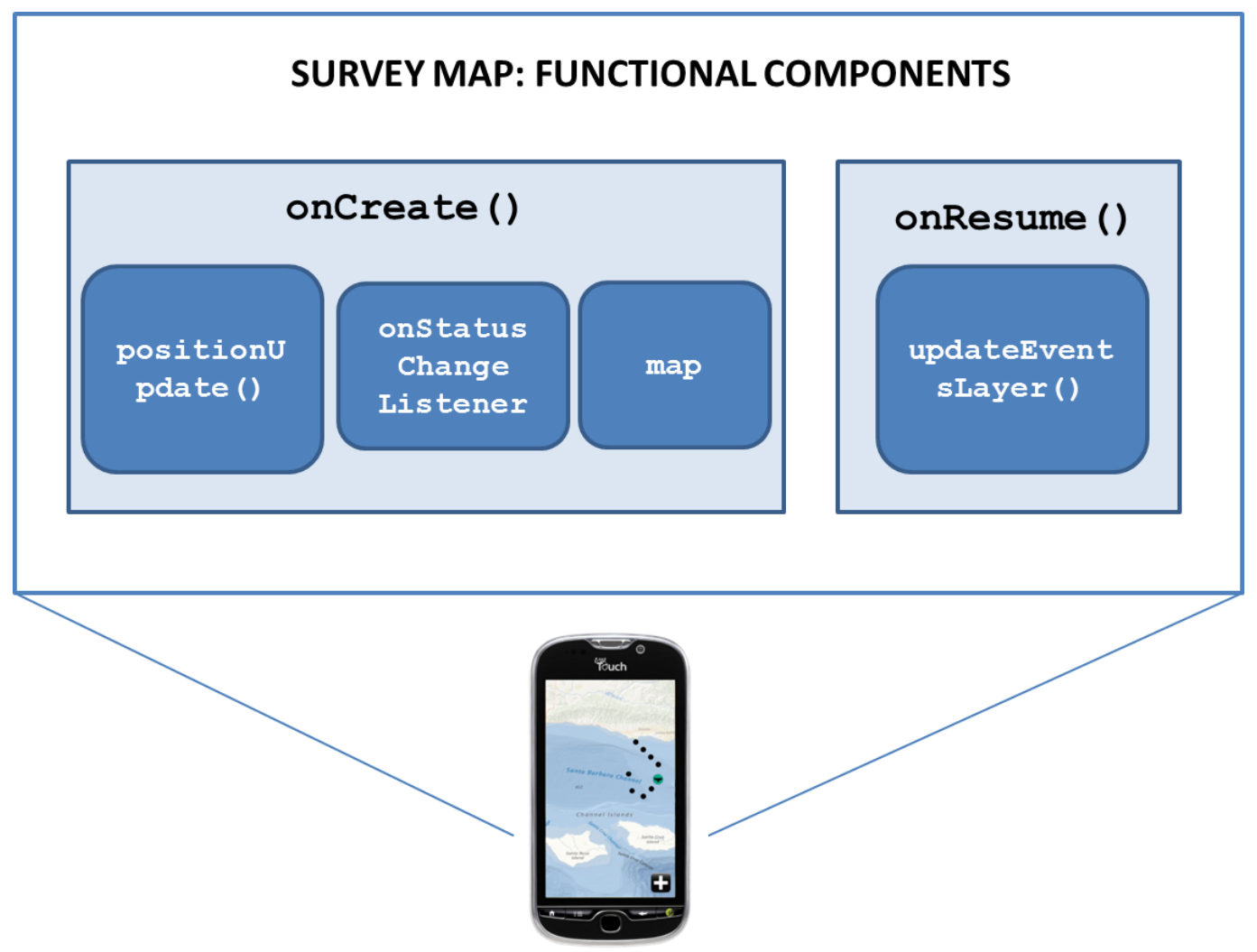

\section{Figure 6-7: Functional components of the Survey Map Activity}

When the Activity is first called by thehome. xml View, the oncreate ( ) function is called. The oncreate ( ) function is an essential part of the Android Life Cycle. The map object is initialized and Esri's Ocean basemap layer is added to it. Additionally in the oncreate ( ) function, the onstatusChange listener is declared. This status change listener handles everything related to the phone's position. It uses the Android location service (Location \& Maps, 2012), which triggers a function when the position has changed. The listener then gets the latitude and longitude from the location service. The latitude and longitude are used to change the position of the location marker on the map. The envelope of the map is then adjusted to extend three miles around the new position.

After the oncreate () method is complete, the onResume () function is called. Within this function, the updateEventsLayer () Method is called. This method uses a static class to create a collection of features with geometry and attributes of each record stored in the local database. It then creates graphics from each of the features in the collection and adds it to the map. Also in the onResume ( ) function, a repeating task is started to automatically record the user's position and draw a black dot on the map every five minutes. This repeating task utilizes Android's Handler class which repeats a particular action with a delayed time increment.

When the user pushes the button to add an observation, the onPause ( ) function is called for the SurveyMap Activity. In this function, the map is paused and the repeating task is stopped so that the position won't be updated during a recording of an observation. 


\subsubsection{Observation Activity}

When the Observation Activity is started, the oncreate () function is called. This Activity is primarily responsible for responding to user actions as they fill out the form, saving, and syncing the data (Figure 6-8).

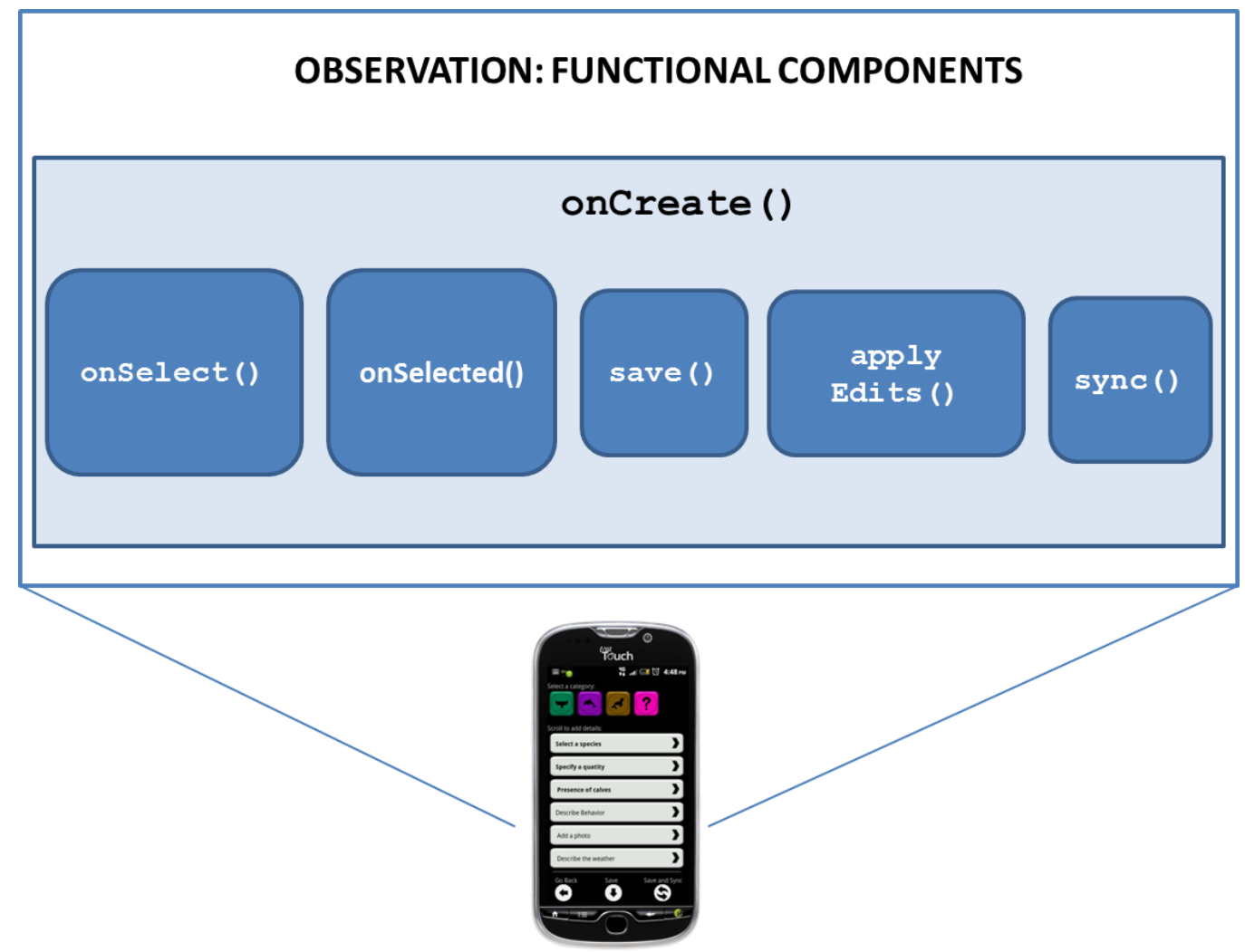

Figure 6-8: Functional components of the Observation Activity

As previously mentioned, the first component of information required is the marine species category. When the user selects a category, the first of this Activity's many onSelected () functions are called. The Species category's onselect () method adjusts the appearance of the detail components required, depending on which of the categories the user selected: whale, dolphin or porpoise, seal or sea lion, unknown (Figure 6-9). 


\section{Detail Components for each Species Category}

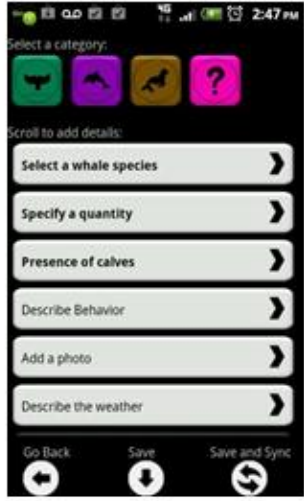

Whale

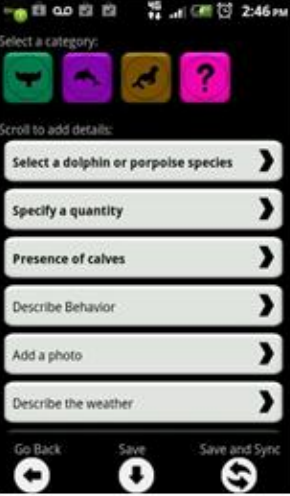

Dolphin

Or

Porpoise

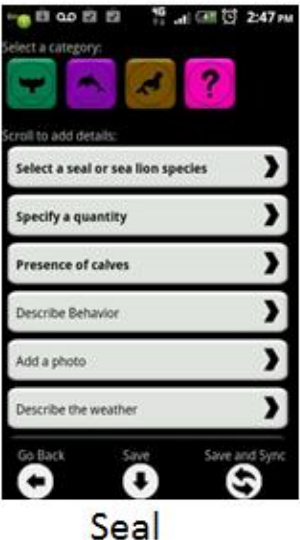

Or

Sea Lion

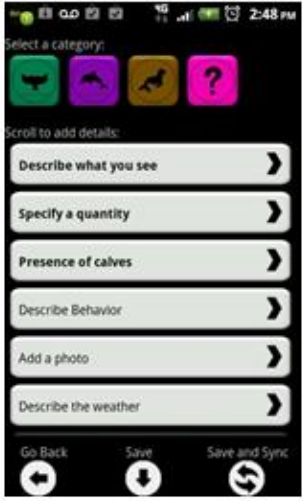

Unknown

Figure 6-9: Detail components provided to the user upon species category selection

After the initial selection, each of the detail components have a similar workflow. On selection, the component's onselect ( ) method is called. These methods build a list of options for the user to select from and populate them in an alert dialog box that is presented to the user (AlertDialog, 2012). The AlertDialog box has a listener associated with it that waits for the user to tap a selection. Once the selection is tapped, the corresponding onselected () method is called. This method is responsible for updating the component detail's description with the choice the user made. This serves as a visual confirmation of what the user is recording (Figure 6-10). 

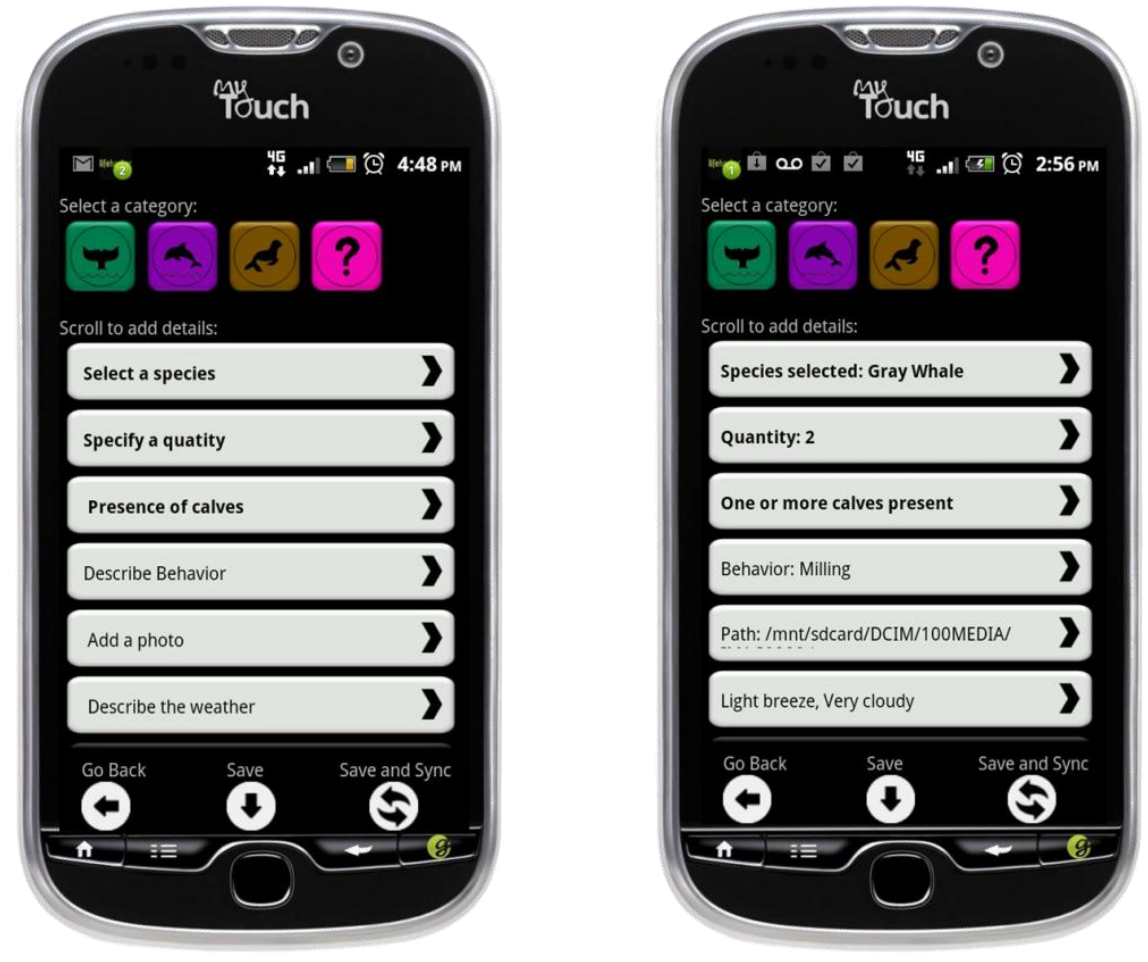

Figure 6-10: Comparison of beginning and ending of the observation form

The third functional component of the Observation Activity is the Save function. This function is called when either the "Save" or the "Sync and Save" button is selected. The first step the method takes is verifying that each of the required pieces of information has been recorded. Afterwards, each of the record's attributes is stored in the local database using methods defined in the EventDatabaseManager Activity.

The final component of the Observation Activity is the Sync function. This function is called whenever the "Sync and Save" button is pushed. The first step the function takes is to call the save method previously mentioned. This ensures that the observation is stored locally. The function then creates a graphic with a geometry passed to the Observation Activity from the SurveyMap Activity. The graphic is also assigned the attributes corresponding to the observation details. The graphic is then passed in a server REST API applyEdits ( ) method to the server.

\subsubsection{Events Database Manager Activity}

The EventsDatabaseManager Activity is responsible for interfacing between the other activities and the database in which the events are stored (Ehrenstein, 2011). The functional components of this Activity are illustrated in Figure 6-11. In the oncreate ( ) method, a new table is created that matches the events feature class schema if one doesn't exist. This should only occur on the initial installation of the application. The open () and close () methods can be called by other activities and are responsible for opening and closing the table so that actions can be performed on it. 
Both of these methods are called by both the SurveyMap and the Observation Activities.

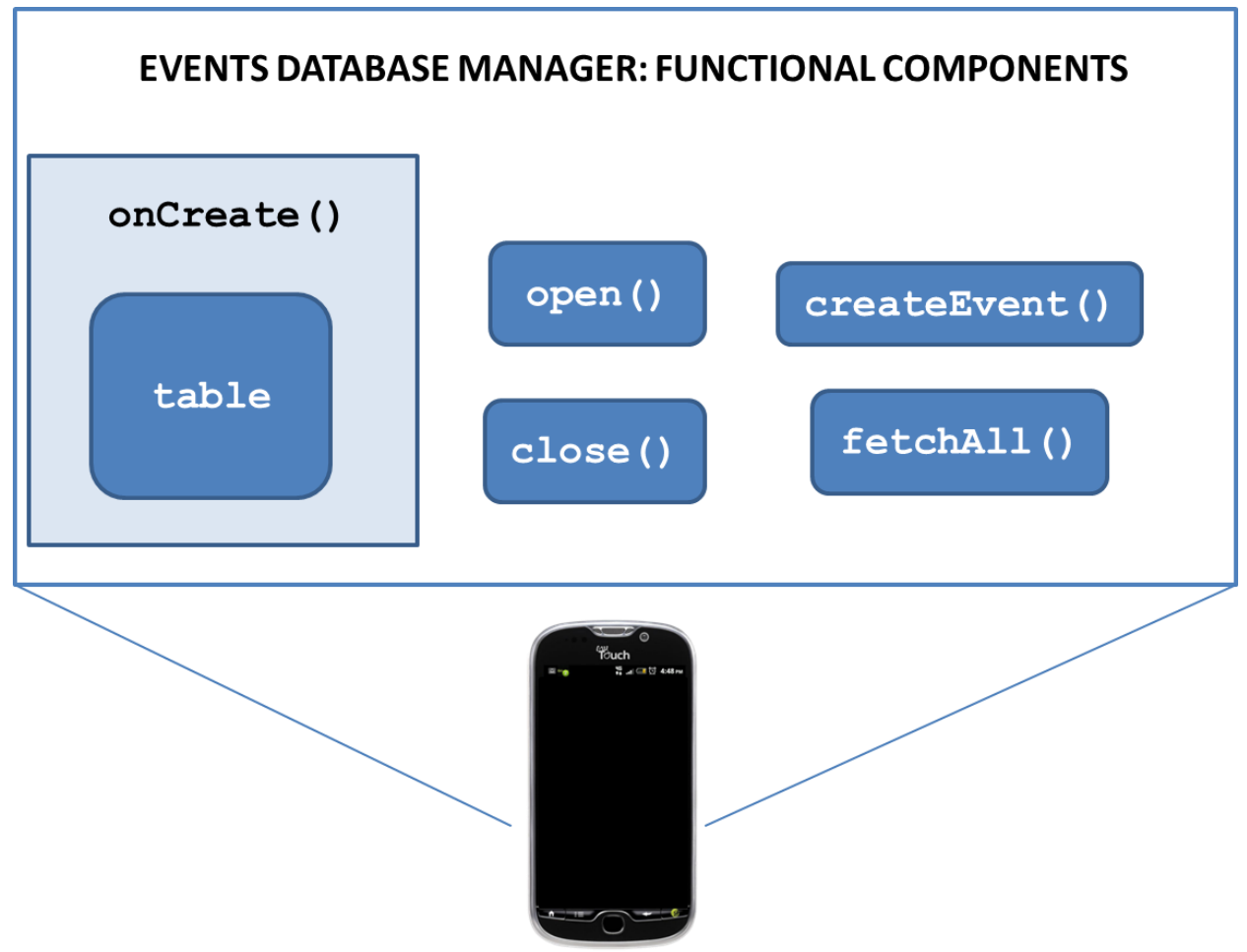

Figure 6-11: Functional components of the Events Database Manager Activity

The fetchAll () method is responsible for querying the table and returning all attributes for every record in the database. This method is called by the SurveyMap Activity. The createEvents () method creates a contentValue object and fills it with the attributes sent to it by the Activity that called the function. It then creates a new record in the database and populates it with the incoming attributes.

\subsection{Summary}

The mobile application consists of three different Views: home.xml, surveyMap.xml, and observation.xml. Each View has a corresponding Activity, and there is an additional Activity for interfacing with the database stored locally on the device. Each Activity has several functional components that are essential to the application.

Both the mobile and web applications play a fundamental role in the project solution. As previously mentioned, the web application allows both users who are actively collecting observations and those that are inactive and simply interested in exploring the data. Active users have access to advanced query tools, an observation submission form, and a data download tool. This mobile application provides a connectivity-hybrid solution for connected editing with data stored locally for future development of a disconnected editing solution. It complements the web application in that it provides a tool for allowing users to submit observations directly from the field. 


\section{Chapter 7 - Implications of Volunteered Geographic Information Software Development}

With the web and mobile applications designed and developed, their implications should be considered. This chapter revisits some of the topics discussed in Chapter Two to understand the effects and implications of the system developed for this project, with an emphasis on the mobile application. Section 7.1 discuses techniques for ensuring enough quality data for scientific research using a VGI approach. Section 7.2 describes how spatiotemporal analysis can be used to identify patterns in VGI data and the differences between this project's data model and the ArcMarine model. Section 7.3 is dedicated to the connectivity issues with the current workflows and toolsets for mobile development. Lastly, Section 7.4 discusses the mobile application user interface and ideas for making it more engaging. The chapter closes with concluding remarks in Section 7.5.

\subsection{Considerations for Volunteered Geographic Information and Science}

To use VGI data in research, the right questions need to be asked of the right people; and enough people have to be asked those questions. The importance of this was discovered during a field test of an early version of the mobile application. Applications that are intended for use by the general public need to frame questions in ways that are easy to understand. The application was originally designed to ask the user how many calves were present with a group of mammals. Counting the number of animals present is a simple task when a group of whales is being surveyed. This is because whales tend to travel in groups of 1-4. However, determining the number of calves present in a group of dolphins is much more difficult because they travel in larger groups of 10-500. Rather than asking the user how many calves were present, the application was changed to simply ask whether there were calves present or not. The true/false response of calf presence can still be used to understand mammal behavior without an exact number.

This was an example of an easy change, which framed the question for a member of the general public. There are some cases in research when the questions cannot be simplified, and therefore should be reserved for trained users. In these cases, user accounts with varying permission levels can be created. For example, an expert user could have access to more involved questions whereas a public user could have access to general questions.

During this project, the application was tested on a single mobile device. In order to collect enough data for scientific research, the application would have to be deployed on a much larger scale (i.e.- Google Play Application Store). In doing so, the design of the system infrastructure would need to be reevaluated. Chapter Two discussed some citizen science-based web applications deployed using open source technologies.

Unfortunately, it is difficult for small organizations to "set up a web server, learn open source (free) web-development Content Management Systems, and ultimately, create, design, and maintain their own website to support their own citizen science program needs," (Newman, Graham, Crall, \& Laituri, 2011, p. 1853). 
Esri has recently developed solutions for multi-scale deployment, as an alternate to open source technology. One solution is ArcGIS Online for Organizations, which gives organizations a mapping platform for sharing maps and editing through feature services. Another solution is ArcGIS Server on Amazon Cloud. Both of these solutions are flexible in terms of the number of users with access. They also do not require personnel to maintain the physical servers that all of the data lives on. Unfortunately, each of the Esri alternatives is expensive. The client would need to apply for an Esri Conservation grant to utilize the technology.

\subsection{The Use of VGI in Marine Research}

Chapter two discussed methods that are currently used in the field of marine research for storing and analyzing data. ArcMarine was described as a data model for storing a wide range of data in an efficient manner for analysis. However, its design demonstrated that it was not intended for volunteered geographic information. The data model would need to be modified before it could be used for the project by eliminating most of the tables, and adding new tables and necessary attributes. The conceptual database designed for this project was similar to ArcMarine in that it associated non-spatial elements (such as an observer or survey) to spatial elements (such as an event). In this respect, the survey table of this project's conceptual model was similar to the survey info table in the ArcMarine data model. Similarly, this project's events feature class is similar to the ArcMarine location series feature class.

Regardless of the similarities in the feature classes and tables, the models diverged during the design of the logical model. Because of the nature of Web and Mobile GIS projects, the conceptual model was consolidated into a flat file structure to improve the speed of queries performed on the database. This made ArcMarine an unrealistic data model for the project's scope.

A study that performed spatio-temporal analysis on Flickr photos found that social media data in conjunction with geovisualization methods could be used to understand mobility and social dynamics in urban systems (SAGL, Resch, Hawelka, \& Beinat, 2012). Similar analysis could be performed on marine mammal observations contributed using a VGI approach. Without taking this into account, improper assumptions and assessments could be made during analysis. Spatio-temporal techniques, including those in ArcGIS tool suite can help understand these space-time clusters. For example, the Grouping Analysis tool can group features based on attributes and with optional spatial/temporal constraints. Similarly, the Cluster \& Outlier Tool uses Local Moran's I statistic to identify statistically significant hot spots, cold spots, and spatial outliers. Tools like this could help determine if the same animal is likely being reported several times by different users. They could also be used to identify groups of events that belong to a particular trip and user.

\subsection{Connectivity in VGI Web and Mobile GIS Applications}

There were a couple of significant obstacles faced during the course of the project, both of which pertain to connectivity of the mobile application. Providing a disconnected editing experience using the current ArcGIS Runtime Software Development Kit (SDK) for Android was challenging. This is because the kit does not include the necessary tools 
for seamless disconnected editing. A work around was created for storing data in a local SQLLite database on the phone. However, the application does not attempt to submit observation data more than once. This is not ideal as users do not always have connectivity. This also forces the user to submit observations as they record them rather than retroactively at their leisure.

The second issue addressed related to connectivity pertains to the basemaps used in the mobile application. In a true disconnected environment, the user would be accessing basemaps stored locally on their device. The geographic scope was limited for the project to an area that had connectivity. Because of this, Esri online basemaps were utilized. If the application was designed for truly disconnected editing, cached basemaps for the user's location would have to be delivered to the mobile device. If the user requested cached maps through the mobile application, the application could be redesigned to identify the user's location, and download the maps necessary for their region. Because of the nature of cached maps, storing them on the Android device could monopolize a significant amount of memory on the phone. For example, the project's study area requires approximately 4 GB of memory in cached maps.

\subsection{Mobile User Interface Critique}

The four areas of proper user interface design discussed in Chapter Two were simplify the task, reduce memorization, plan for error, and test the usability of the application. The task of submitting observations was simplified in the project by taking the scientific form used in the client's research and turning it into an easily accessible form in either a mobile and web application. Reducing memorization was handled by providing the user with a list of options to choose from rather than asking them to manually enter the requested information. A feedback system was designed in the mobile application in planning for error. After completing each element in the observation form, the user is presented with a visual confirmation of their selection. This feedback system, allows them to change their selection if desired.

The usability of the mobile and web applications were not tested over the course of this project. There are several components of the user interface that could be improved to make the system more engaging. One approach to this is to game-ify the experience. Adding game elements to the applications could provide the users with opportunities for collaboration and a feeling of belonging to something greater than themselves (McGonigal, 2011). Some game elements include feedback loops that could tell the user information such as how many observations they've submitted to the community average. Another game element for engaging users is to challenge them intellectually. Users could be challenged by competing or working as a team to collect data and answer trivia questions. This method also encourages collaboration among the application users. If users don't already have a team to collaborate with, a notification system could be used to inform users if they are in close proximity of other users.

\subsection{Summary}

In conclusion, Section 7.1 demonstrated how the right questions need to be asked to the right people. It also discussed how expanding VGI projects to collect enough data for research is either time consuming or expensive. Section 7.3 explored the differences 
between the data model developed for this project and the existing ArcMarine model. It also described how GIS can be used to identify spatiotemporal patterns in data. In Section 7.3 the disadvantages surrounding connectivity and Mobile GIS. A better toolset is needed for disconnected editing with the Runtime SDK for Android. Additionally, better workflows are needed for handling offline basemaps. Finally, Section 7.4 discussed the design of the mobile user interface and how error was accounted for, tasks were simplified, and memorization was reduced. Testing of the mobile application usability still needs to be performed, and the user interface could be improved using game elements to make it more engaging. 


\section{Chapter 8 - Conclusions and Future Work}

The challenge that the client faced was how to generate a high volume of quality data for her long-term study on marine mammal migratory behavior and human impact. In order to reduce the cost and effort required in the data collection process, Stelle decided to explore a VGI approach to collecting this data, which incorporates the collection of data by both researchers and members of the general public. The proposed solution consisted of web and mobile applications and a geodatabase for storing observations collected by researchers and members of the general public.

A conceptual model was used to describe the client's business model and logical models for both the server and client side were designed. The server side logical model consisted of a single feature class for holding all submitted events and an associated attachment table to hold all evidence, such as photos. The client side logical model consisted of a single table in a SQLLite database on the mobile device. All evidence was stored as a path to its location in the Photo Gallery on the Android device. Domains and subtypes were incorporated into the server side model to ensure data integrity.

The web application was designed for the user to visualize all data on a map and query the data based on the type of species, date range, and event type. It was also designed to allow users to download selected data in a shapefile format. The web application interface includes a form for submitting observations upon login to the system.

The mobile application was designed to allow users to visualize their whale watching trip and corresponding recorded observations. This is done by rendering a new position update on a map every five minutes. A form was created that allows users to submit new observations and corresponding evidence. The user can choose to associate their observations with a user account or submit them anonymously. The data associated with the observations and position updates are stored locally on the device and synced with the server when submitted. Upon which, a new symbol is rendered on the map for their observation.

The purpose of this project was to demonstrate the capability of the system with proofs of concept. During development of the proofs of concept, some recommendations were determined pertaining to the user experience and expanding the project to a larger audience.

The first suggestion for improving the user experience is to give users more control over their data. On the mobile application, the user should be able to retroactively submit observations after a trip. Currently, the user has a single chance to submit the observation. In some cases, the user may also desire to edit their observations. For example, they may have made a mistake in the details of an observation or forgot to submit a photo with the observation. Editing capabilities of observations should be added to the web application interface. This capability would, for example, allows users to add photos to previously submitted observations.

There are other suggestions for improving the user interface and making the applications more engaging. The applications could include game elements such as feedback loops, collaboration, and competition to give the user a feeling of belonging to something greater than themselves. Feedback loops could tell the user how many 
observations they've submitted compared to the others in the community. Specific examples of how these elements could be incorporated are discussed in Chapter Seven.

In order for the application to be deployed to a wide audience, several considerations need to be taken into account. Currently, the applications are only designed to allow users to select from animals in southern California. The available species list would need to be expanded to a much larger scale. With a larger geographic scope, the mobile application would need to be capable of working in completely disconnected environments. To do so, basemaps would need to be cached and available for download by users in these environments.

As the above suggestions are incorporated, it is essential that the applications are tested on small user groups before deploying at a large scale. Testing will provide important feedback on the user experience. These suggestions for future work will produce a system that is ready for use by a wide range of users.

In summary, this project demonstrated how volunteered geographic information could be used alongside Web and Mobile GIS to manage marine mammal observations. Several suggestions for improvement have been identified for further development. Upon deployment, the data collected using the applications can play a key role in understanding human impact on, and behaviors of, migrating marine mammals. 


\section{Works Cited}

Activity. (2012, 10 12). Retrieved 10 14, 2012, from Android Developer: http://developer.android.com/reference/android/app/Activity.html

AlertDialog. (2012, 10 12). Retrieved 10 14, 2012, from Android Developer: http://developer.android.com/reference/android/app/AlertDialog.html

Android 2.3.3 APIs. (2012). Retrieved 2012, from Android Developer : http://developer.android.com/about/versions/android-2.3.3.html

Android Developer Reference. (2012). Retrieved 10 9, 2012, from Intent: http://developer.android.com/reference/android/content/Intent.html

Aberley, D., \& Sieber, R. (2002). PPGIS.net Open Forum on Participatory Geographic Information Systems and Technologies. Retrieved 12 2012, from About PPGIS: http://www.ppgis.net/ppgis.htm

Aguilar, M. (2012, July). Gizmodo.com. Retrieved July 2012, from Only 10-Percent of Androids Have Ice Cream Sandwich After Eight Months: http://gizmodo.com/5923143/only-10percent-of-androids-have-ice-cream-sandwich-after-eight-months

Apple. (1987). Human Interface Guidelines: The Apple Desktop Interface. Addison-Wesley Publishing Company Inc: New York.

Bauer, J. R. (2012, 6). Assessing the Robustness of Web Feature Services Necessary to Satisfy the Requirements of Coastal Management Applications. MA Thesis. Retrieved 102012 , from Oregon State University Library: http://ir.library.oregonstate.edu/xmlui/bitstream/handle/1957/30062/BauerJennifer2012.p df? sequence $=1$

Best, B., Halpin, P., Fujioka, E., Read, A., Qian, S. S., Hazen, L., et al. (2006). Geospatial Web Services Within a Scientific Workflow: Predicting Marine Mammal Habitats in a Dynamic Environment. Ecological Informatics.

Connors, J. P., Lei, S., \& Kelly, M. (2011). Citizen Science in the Age of Neogeography: Utilizing Volunteered Geographic Information for Environmental Monitoring. Annals of the Association of American Geographers, Forthcoming 2012.

Davidson, A. D., Boyer, A. G., Kim, H., Pompa-Mansilla, S., Hamilton, M. J., Costa, D. P., et al. (2012). Drivers and Hotspots of Extinction Risk in Marine Mammals. Proceedings of the National Academy of Sciences of the United States of America, 3395-3400.

Dojo. (2011). Dojo toolkit. Retrieved 10 1, 2012, from Dijit Documentation for Version 1.6: http://dojotoolkit.org/reference-guide/1.6/dijit/index.html\#dijit-index

Ehrenstein, C. (2011). Android App Development \& Design. Learn By Video. Peach Pit Press. Esri. (2011, Dec 14). Android Runtime SDK for Android. Retrieved Feb 22, 2012, from Esri: http://resources.arcgis.com/content/arcgis-android/sdk

Esri. (2012, Feb 1). What is a Feature Service? Retrieved Feb 20, 2012, from Esri: http://help.arcgis.com/en/arcgisserver/10.0/help/arcgis_server_dotnet_help/index.html\#// 009300000020000000

Esri. (n.d.). ArcGIS Server Training Seminars. Retrieved Feb 20, 2012, from Esri: http://training.esri.com/campus/catalog/ServerSeminars.cfm

Foley, M. M., Halpern, B. S., \& Micheli, F. (2010). Guiding Ecological Principles for Marine Spatial Planning. Marine Policy, 955-966.

Francica, J., \& Schutzberg, A. (2011, Sep 13). Top Skills Needed to be Successful in a GIS Career. Directions Magazine Podcast. Podcast retrieved from iTunes. 
Fu, P., \& Sun, J. (2011). Web GIS Principles \& Applications. Redlands: Esri Press.

Geospatial Innovation Facility. (2012). About OakMapper. Retrieved from OakMapper Sudden Oak Death: http://www.oakmapper.org

Glennon, A. (2011). Geyser Notebook. Retrieved February 14, 2012, from Android Market: https://market.android.com/details?id=net.geysers.app.geysernotebook

Glennon, A. (2012, February 15). personal communication. (M. King, Interviewer)

Goodchild, M. F. (2007). Citizens as Sensors: the World of Volunteered Geography. GeoJournal, 211-221.

Google. (2012). Android. Retrieved Feb 23, 2012, from Android Developers: http://developer.android.com/index.html

Halpern, B. (2008). A Global Map of Human Impact on Marine Ecosystems. Science, 948-952.

Halpin, P. (2004). NOAA Office of Science \& Technology / EcoGIS: GIS Tools for Ecosystem Approaches to Management. Retrieved August 15, 2012, from Spatial Analysis Needs for Marine Ecosystem Management: Habitat Characterization, Spatio-Temporal Models and Connectivity Analysis Frameworks: http://www.st.nmfs.noaa.gov/ecogis/Workshop2004/Documents/Halpin_presentation.pdf

Jones, J. S., \& Ganey, S. (2009). Building the Legal and Institutional Framework. Ecosystembased Management for the Oceans, 162-179.

Location \& Maps. (2012, 10 12). Retrieved 10 14, 2012, from Android Developer: http://developer.android.com/guide/topics/location/index.html

Lloyd, C. (2012, September 20). Android Community. Retrieved October 1, 2012, from Verizon sees more Android activations than all other carriers: http://androidcommunity.com/verizon-sees-more-android-activations-than-all-othercarriers-20120920/

Mandel, T. (1997). The Elements of User Interface Design. New York: John Wiley \& Sons.

McGonigal, J. (2011). Reality is Broken: Why Games Make Us Better and How They Can Change the World. New York: The Penguin Press.

National Ocean Service. (2011, 1 6). Marine Mammal Sightings Database. Retrieved 1016 , 2012, from Channel Islands National Marine Sanctuary: http://www.cisanctuary.org/mammals/

Newman, G., Graham, J., Crall, A., \& Laituri, M. (2011). The art and science of multi-scale citizen science support. Ecological Informatics, 218-226.

Newman, G., Zimmerman, D., \& Crall, A. (2010). User-friendly web mapping: lessons from a citizen science website. International Journal of Geographic Information Science, 18511869.

NOAA. (2011, 7 31). NOAA Coastwatch: West Coast Regional Node. Retrieved 10 16, 2012, from Coasta watch Browser: http://coastwatch.pfeg.noaa.gov/coastwatch/CWBrowser.jsp

Norman, D. A. (1988). The Design of Everyday Things. New York: DoubleDay.

O'Malley, R. (2010, 9 15). Ocean Productivity. Retrieved 10 16, 2012, from http://www.science.oregonstate.edu/ocean.productivity/

Pelton, M. R., \& Manen, F. T. (1996). Benefits and Pitfalls of Long-Term Research: A Case Study of Black Bears in Great Smoky Mountain National Park. Wildlife Society Bulletin, 443-450.

Pian, S., \& Menier, D. (2011). The use of a geodatabase to carry out a multivariate analysis of coastline variations at various time and spave scales. Journal of Coast Research Special Issue 64, 1722-1726. 
Pompa, S., Ehrlich, P. R., \& Ceballos, G. (2011). Global Distributions and Conservation of Marine Mammals. Proceedings of the National Academy of Sciences of the United States of America, 13600-13605.

Ruckelshaus, M., Klinger, T., Knowlton, N., \& DeMaster, D. P. (2008). Marine Ecosystembased Management in Practice: Scientific and Governance Challenges. BioScience, 5363.

SAGL, G., Resch, B., Hawelka, B., \& Beinat, E. (2012, 3 7). From Social Sensor Data to Collective Human Behaviour Patterns - Analysing \& Visualising Spatio-Temporal Dynamics in Urban Environments. Retrieved 10 23, 2012, from GIS Point: http://gispoint.de/index.php?id=5\&tx_browser_pi1\%5BnewsUid\%5D=682\&cHash=2082 90d911

Steiniger, S., Neun, M., \& Alistair, E. (n.d.). University of Minnestota - Spatial Database and Spatial Data Mining Research Group. Retrieved Feb 23, 2012, from Foundations of Location Based Services: http://www.spatial.cs.umn.edu/Courses/Spring10/8715/papers/IM7_steiniger.pdf

Stelle, L. (2008). Activity budget and diving behavior of grey whales (Eschrichtius rubustus) in feeding grounds off coastal British Columbia. Marine Mammal Science Vol 24. No 3, 462-478.

Stelle, L. (2012, Feb 10). Data Integration: Marine Mammals and Their Ecosystems Citizen Science "Whale mAPP" [Powerpoint slides]. Redlands, California: Unpublished.

The Cornell Lab of Ornithology. (2011). eBird. Retrieved Jan 31, 2012, from eBird: http://ebird.org

Timoney, B. (2012, 8 1). How the Public Actually Uses Local Government Web Maps: Metrics from Denver. Retrieved 10 1, 2012, from Map Brief:

http://mapbrief.com/2012/08/01/how-the-public-actually-uses-local-government-webmaps-metrics-from-denver/

Tsou, M.-H. (2004). Integrating Mobile GIS and Wireless Internet Map Servers for Environmental Monitoring and Management. Cartography and Geographic Information Science, 153-165.

University of Washington Sea Grant Institude. (2011, 8 11). Impacts and Outcomes of Mature Coastal Web Atlases: California. Retrieved 10 2012, from YouTube: http://www.youtube.com/watch?v=cLPq65Rj0uA

UW Center for Game Science. (2011). Fold it: Solve Puzzles for Science. Retrieved Jan 31, 2012, from Homepage: http://fold.it/portal/

Viswanathan, P. (2012). About.com. Retrieved October 1, 2012, from Android OS Vs. Apple iOS - Which is Better for Developers?: http://mobiledevices.about.com/od/kindattentiondevelopers/tp/Android-Os-Vs-Apple-IosWhich-Is-Better-For-Developers.htm

Wagtendonk, A. J., \& De Jeu, R. A. (2007). Sensible Field Computing: Evaluating the Use of Mobile GIS Methods in Scientific Fieldwork. Photogrammetric Engineering \& Remote Sensing Vol. 73, No. 6, 651-662.

Walker, D. A., Halfpenny, J. C., Walker, M. D., \& Wessman, C. (1993). Long-term Studies of Snow-Vegetation Interactions. BioScience, 287-301.

Wright, D., Blongewicz, M., Halpin, P. N., \& Breman, J. (2007). Arc Marine. Redlands: ESRI Press. 


\section{Appendix A. Geodatabase Domains}

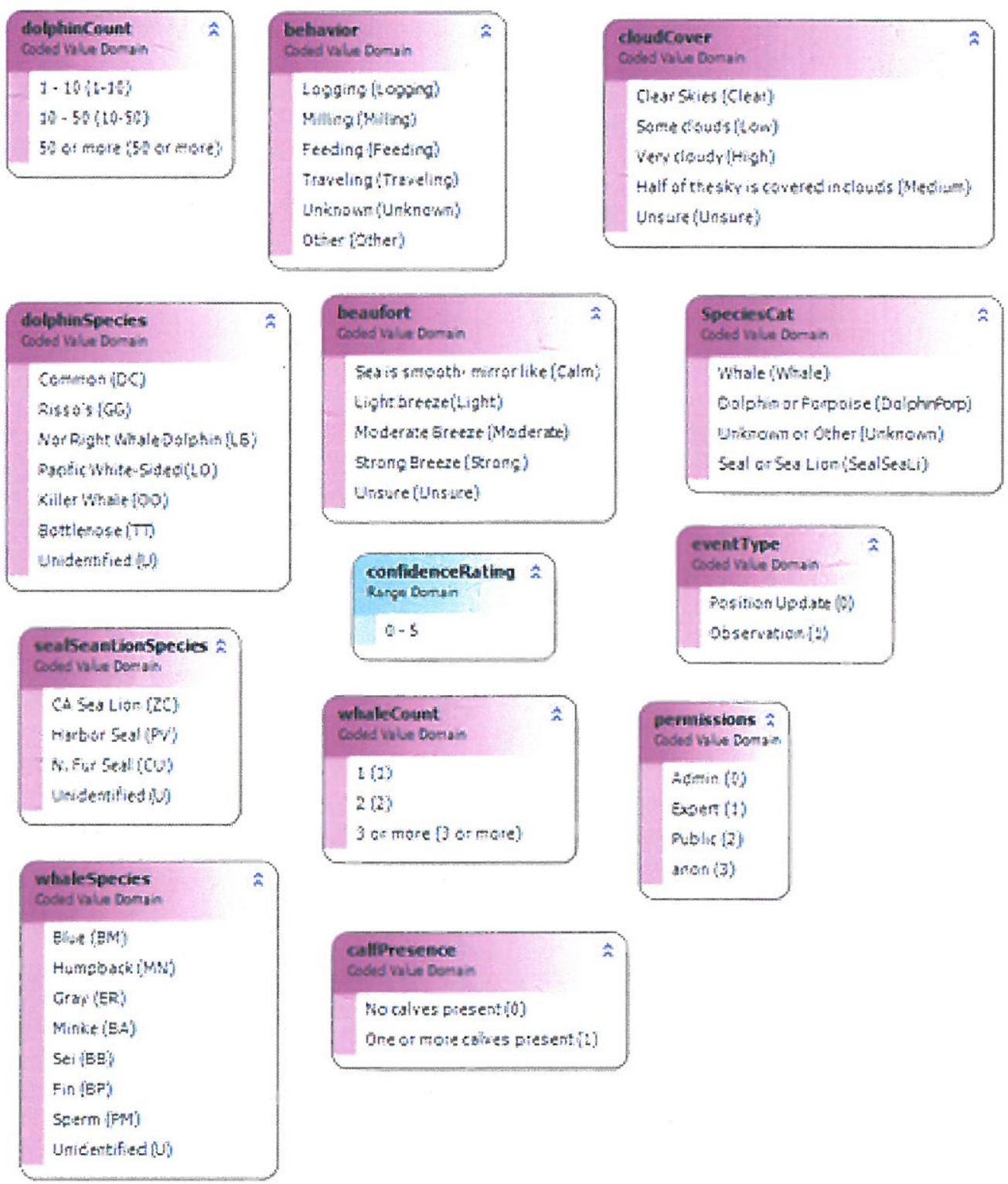



Appendix B. Mobile Application Activities Code 

Observation.java

1 package edu.gis.spatial.redlands.edu.Cohort21.melodi_king;

2

3 import java.io.File;

65

66 public class Observation extends Activity \{

67 int SELECT_PICTURE = 1 ;

68 public double locy;

69 public double locx;

70 public String userID;

71 int $i=999$;

72 public boolean catpicked = false;

73 // private Spinner speciesType;

74

75

76

77

78

79

80

81

82

83

84

85

86

87

88

89

90

91

92

93

94

95

96

97

98

99

// database object

private EventsDBManager mDbManager;

// initial/default variable values

private String category = "Unknown or Other";

private String speciesType = "Unidentified";

private String count = "No calves present";

private String notes $=" "$;

private String behavior = "Unknown";

private String calves = "No calves present";

public String seaString $="$ ";

public String weatherString = " ";

private Cursor mCursor;

private RatingBar ratingBar;

String txtRating;

public boolean speciesPicked = false;

public boolean countPicked = false;

public boolean calvesPicked = false;

public int catInt;

private int rateInt $=2$;

// no longer using credentials, can delete

// UserCredentials credentials = new UserCredentials();

// ArcGISFeatureLayer fLayer = new

//

ArcGISFeatureLayer("http://gis.spatial.redlands.edu/ArcGIS/rest/services/melodi_king/ events/FeatureServer/1",

100 // MODE.SNAPSHOT, Usercredentials(credentials));

101 ArcGISFeatureLayer fLayer = new ArcGISFeatureLayer (

102 "http://gis.spatial.redlands.edu/ArcGIS/rest/services/melodi_king/events/

FeatureServer/1",

103

104

105

MODE.SNAPSHOT);

int permissions $=2$;

String layerDefinition $="\{\backslash$ "currentVersion $\backslash ": 10.02 ; \backslash$ id $\backslash ": 1, \backslash$ "name $\backslash ": \backslash$ "events

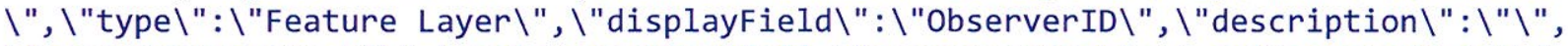

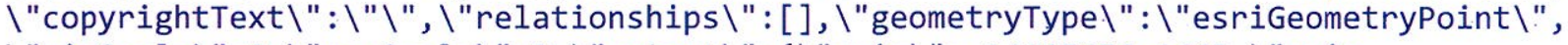

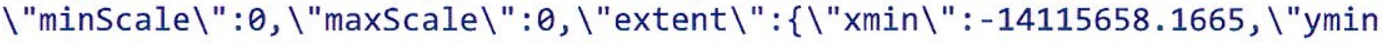

$\backslash ": 3240128.2558, \backslash " x \max \backslash ":-13061473.3222, \backslash " y \max \backslash ": 4321833.7614, \backslash "$ spatialReference $\backslash ":$

$\{\backslash$ "wkid $\backslash ": 102113\}\}, \backslash$ "drawingInfo \":\{\"renderer $\backslash ":\{\backslash$ "type $\backslash ": \backslash$ "uniqueValue $\backslash "$,

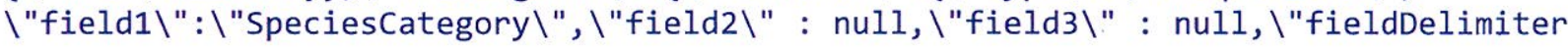


Observation.java

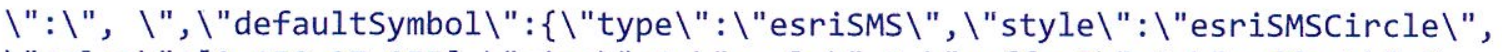

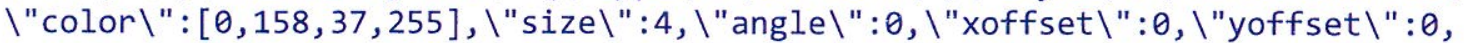
$\backslash$ "outline $\backslash ":\{\backslash$ "color $\backslash ":[\theta, \theta, \theta, 255], \backslash$ "width $\backslash ": 1\}\}, \backslash$ "defaultLabel $\backslash ": \backslash " \backslash$ u003call other

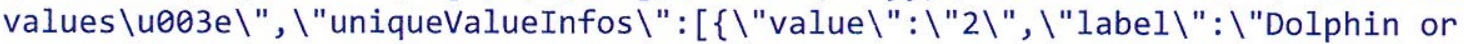

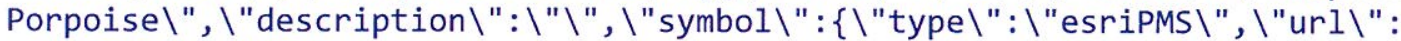
\"B698AF73\",\"imageData\": \"iVBORwOKGgoAAAANSUhEUgAAABgAAAAZCAYAAAArK +5dAAAAAXNSROIB2cks fWAAAAlWSFIzAAAOXAAADsQB1SsOGWAAAWFJREFUSInllwtIk2EUx397t/mmstSZS 20i3iorDbbuYhQ1kUFGYGFEd6w+FEVpRZFhSR

+EUAlBsQhaQh/EriSRYjUUwyS3LphamJdZ5BtUZi/uOpcarc1pQRBOPj1wzvP/PeccznIU/GVT/RcAtZE1ux NISQsiJFSFSpAZ/tSNtbeJmnbABNj/CJBN/towUVe4UF496zEN6rksJZQIt38bBc011C5rFE0Vsiw3/xZgl3 j2mF6edSXZnq/po4ty91HITQ9ACFMW7+D0giR5XtB59oYDtyYEyOPiO1GeXJDMfBHAzDUASjjIevbgYBSAqe hJxKhMJyv7K1+04lA38GIcgEsxQnmFkVUiwHsGqOUCABJdVHHYI1pLLPspI40crA6ahh5Ss9cvoFC8kR8kz9 E5sNNCHSaKfSXpNokeTpFFEXcCVrJ1ZldUU6rNZrOMCfggS1uSMHCPai5wXEtQSyKbOcx0jIShQ2aEFuqopp g8KhZim5wJfgCBTIoRUFJLiZd4NvlkspMgND

+VQI2RFVRWgGc0BxpYob9Nx9glUqAMduJEWUYFSCDFQ/yHWTBzICuEEEEWYcE/

+3w12fGeAWEG6Wwij1hmEkAgfbykHhORXBNFnDvaiZPpGJhKDACt3PMYOi+Ag9GhYT5GHqXK47WXJLOdMzh + GVoBWS3UXMUQfYN

+ARFafYNLYpOvUihQoELtnfR366ez36Ktq0HyA4iWkk484s5GPUnKH6IALlzus51RnyArD9okSXriN4Mcpro uOZovWSXzTjUiGrToSaSdRvTMYArRWHIIFPHoikGeqxhYzjt637TTePXXPZ

+rYraUltvE7blveTVPSyQdtJFAKiN8wYqZSOKwY8eKmcWsoY36z328LHpCg3VCgAwUzkqeLVIimLp5uiGFJY KGUDSEo/veUIBworFwv30QziPXKav1pTXmNs1ltgPIqaTtdAetpeBMDSBQC4Lgwv5XmOEeSRy8e1k

+cRL40pbOuB90LobnQIZPpzze7X/ky/zPAd8AJIX1ZOTUiUWAAAAASUVORK5CYII= \", \"contentType $\backslash "$ :

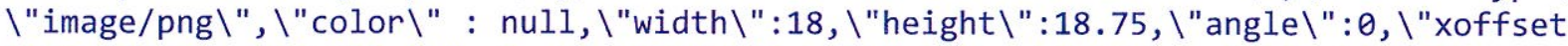

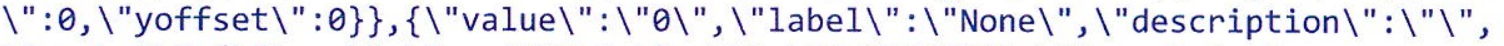

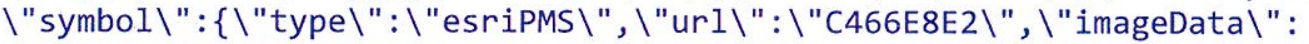

\"iVBORwOKGgoAAAANSUhEUgAAAAwAAAAMCAYAAABWdVznAAAAAXNSROIB2cks fwAAAAIwSFIzAAAOXAAADs QB1SSOGWAAAJ1JREFUKJGtObEKQVEYB/DFQDezDBZ5C6U8AQm7V7CIyWSUhzGZWL2EzAaZ9WWxUPderlLOeM 75nf7/75T8uEr/AHWsOHue7zDH8R0o4oBmam

+ADlo4ZUCSJIUISF90P7TGMAMiYvQlfheVfKT6F1BGLQ/OaBSA0y75DpuImBSALW75Dkv0ZacEV0x5H +sVbdl/2GOGOyfw6jEuiOUB/ZOhYNqEvhsAAAAASUVORK5CYII= \", \"contentType \": \"image/png \",

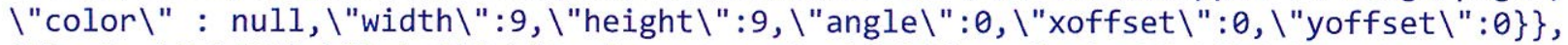

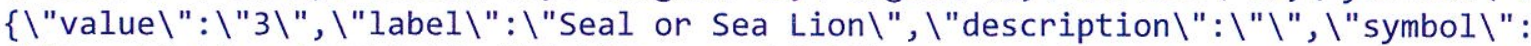

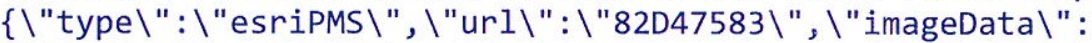

\"iVBORwOKGgoAAAANSUhEUgAAABkAAAAZCAYAAADE6YVjAAAAAXNSROIB2cks fwAAAAIwSFlzAAAOXAAADs QB1S S0GWAAAxVJREFUSInt1V1MU2CYWPF/vZiFFkIOiJRaCREiH5HVr3WgQUKyGDUab0S3i4Vkx10Yw41ZRm K2+HG9hMwtMdkFRi8gJkuWLCxzi1kmk4ABK4qiVJqWwaEIr9oK9NDT010QiaW20C8WE32uznne9zy/5zk5H2 b+hzC/U4ilqVT+oswp7cgzZeVjwajGFiOjQTH+eOC9DVwGtDdGWjw1B9bYtDMNG4uqc7MtlqTF7XD8ebT3Yr $+/ \mathrm{sfu}$ +uKCqau9/Rlqbyts2FFjaal1rc9Ptybdb61qbqj7c5AjmnPnVVwD88trIuf01B21Wvql1yVI6ILwQpW9smpF /hCmscqi1sdTc/mfgEXB/VSQBhq817cI0lzMtAHC138+lAQWArqP1FOVZ940oEeW3B

+L4qsj5I54vyyWKDBmA6GJ sGQAotGVhgKWDm9dXDYWIWkVRhjIiT

+ZmP6t2rM80BHcnRPI1C4sU2q1UOmQPwrsXyIXIRpPL1GYMLa4TEBG

+6/Y15aeez1Not2IykLOrXF7XOZzcRApiNpptL5/H4zpBEeHW

+AzdQ1P4hJqCz8diy8c2u2RPqbkyoaPFAWMCGJ0SdPYHueYTK7etaOylptSs1BczBdF0ZvUExX1jIdp +Hs5Y/EU48paa1X0gPJ8KrYqU5NuvTTyZ

+9T/OLn7UgnqqmSskkRH39KTJQFuh8SCppEAAjPPJm8K2xVIvqUpSF1BzqmeUXG4vqLENPtMZVGDbDPs2VKG S871Ys8DAFo8Dpq31TMYnObGW010HW4FZ7XCCO

+qkzR8e91/qcXT8XRe/fzEbnfS201HIdSoyvfNbqqdMgYD7KxwsrPCyWAgNP63L/DVynqvRACKO/qOhY +4 PXiZFNsqS+Tl/OZ1MvUb1qbsvzcpRP

+Ictqrc0e1kY9BH27yfvTXH1sv94yGmt0u2Wi3WsiWkj/Ec9EYvf5pf2A6fLJzWPzOqlppEYCaY8Rh4J0uo1 vP3plQ2h06qdZiRjZiNmo6kbmoFpyzf3z1h

+viFBBNVycj8iI0/zhwb2m4N4+35vf7HImOfwEYGSjqIxnPkQAAAABJRU5ErkJggg==\", \"contentType

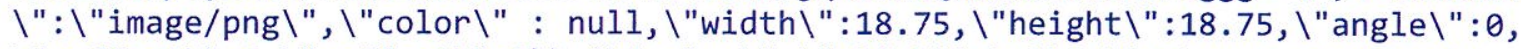

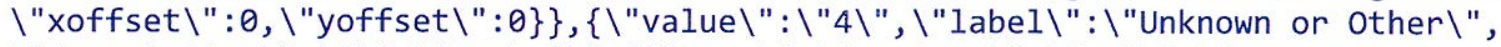

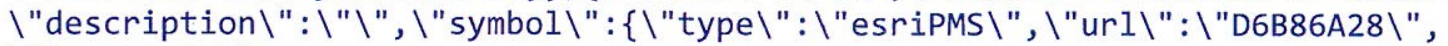

$\backslash$ "imageData\":

\"iVBORwOKGgoAAAANSUhEUgAAABsAAAACCA Pag QB1Ss0GWAAAWNJREFUSIntlktIVFEYx393Rj30jG0OhVLmk8zQNmEa2CLKyt4PKnET05PRykIh0qXFYQ96SU

KWkJUtgooCKOMxlRb2oAiiIArptUhoMYp0o2fMua1mmaveGUuIFn $2 \mathrm{r} 7 / 7 / 3 / 1+9 z u c v 71 \mathrm{~h} /$ MUI 
Observation.java

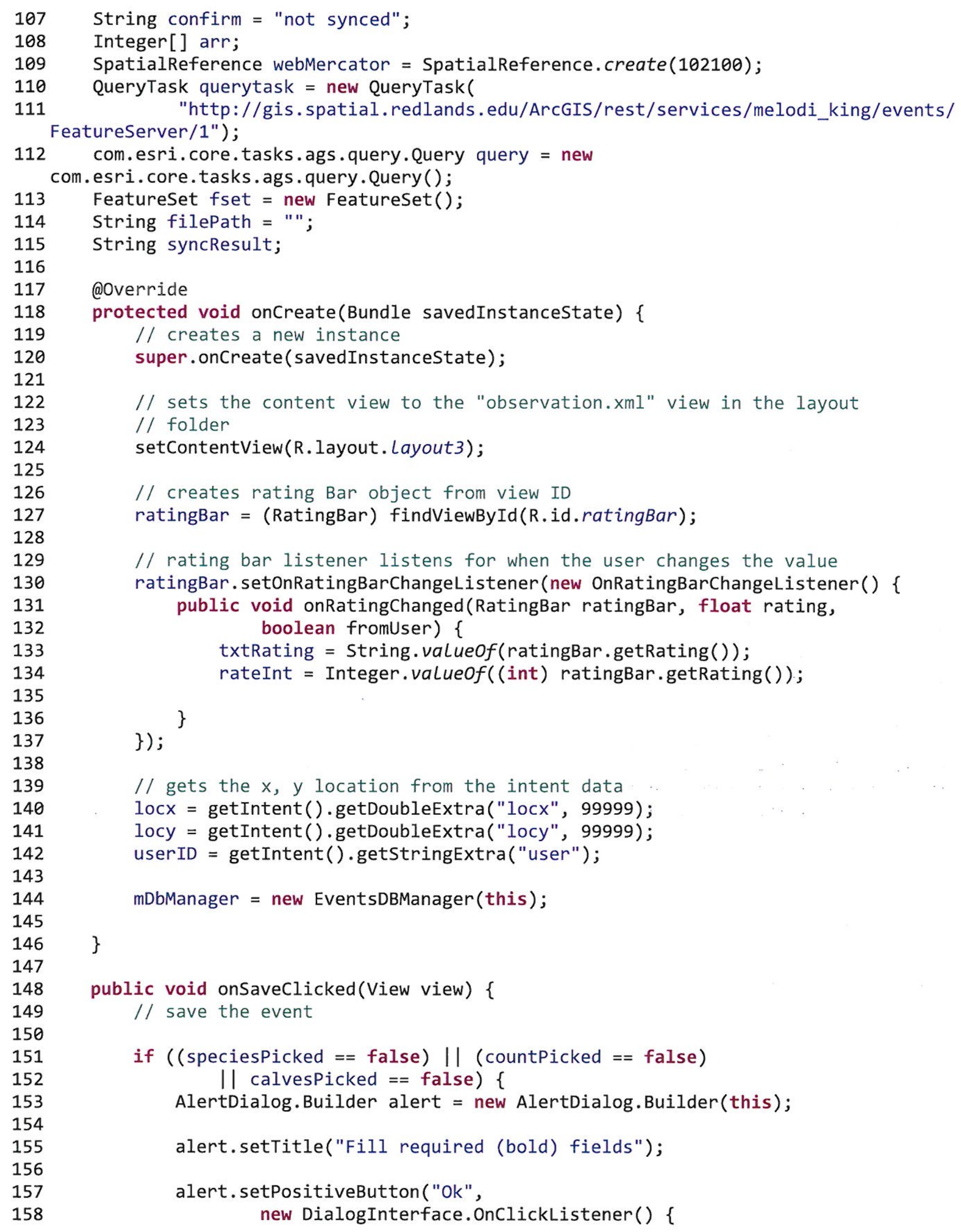




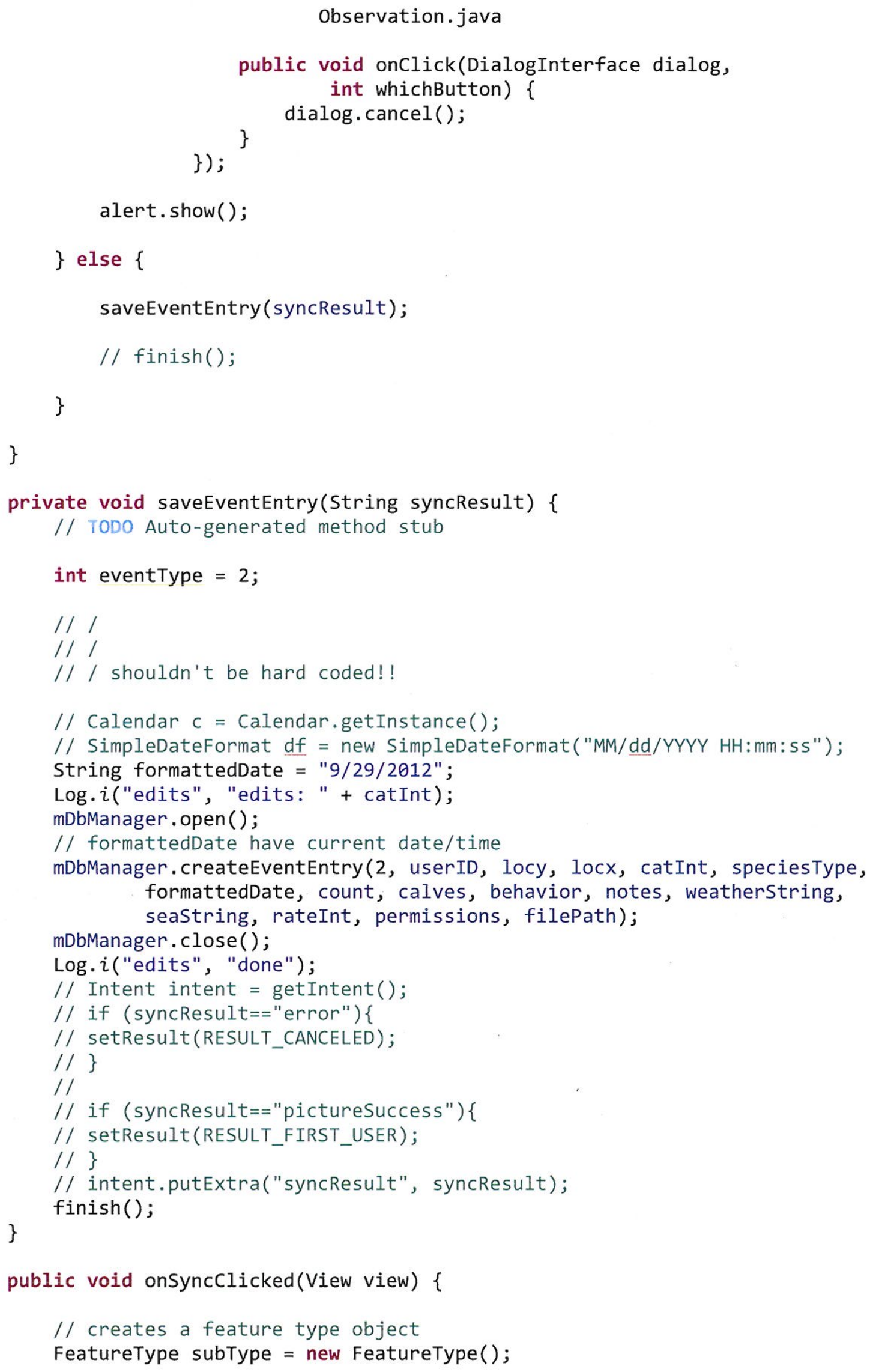

Observation.java 
Observation.java

subType $=$ fLayer $\cdot$ getTypes ()$[$ catInt $]$;

// calls the function that sends the new record to the server applyEdits(GeometryEngine.project(locx, locy, webMercator), subType, fLayer, view);

\section{\}}

public void applyEdits(Geometry geometry, FeatureType subType, final ArcGISFeatureLayer featureLayer, View view) \{

// create a graphic using the type

Calendar rightNow $=$ Calendar.getInstance () ;

// creates graphic so that that the graphic can be sent to the server Graphic graphic = featureLayer.createFeatureWithType (subType, geometry);

// adds attributes to the graphic

Map<String, Object> attr = graphic.getAttributes () ;

attr.put("Number", count);

attr.put("ObserverID", userID);

attr.put("Behavior", behavior);

attr.put("SpeciesType", speciesType);

int calfInt $=1$;

if (calves $==$ "No calves present") \{ calfInt $=0$;

\}

attr.put("CalvesPresent", calfInt);

attr.put("Notes", notes);

attr.put ("CloudCover", weatherString);

attr.put("Beafort", seastring);

attr.put("ConfidenceRating", rateInt);

attr.put("eventType", 1);

attr.put("Permissions", permissions);

attr.put("Date", rightNow);

// create a new graphic with the attributes. attributes are immutable Graphic newGraphic = new Graphic(geometry, graphic.getSymbol(), attr, graphic.getInfoTemplate());

// applies edits

featureLayer.applyEdits(new Graphic [] \{ newGraphic \}, null, null, new CallbackListener<FeatureEditResult[][]>() \{

public void onError(Throwable error) \{

\}

public void onCallback(FeatureEditResult[][] editResult) \{ Log.i("edits", "inside event callback");

if (editResult[0] != null \&\& editResult[0][0] != null $\& \&$ editResult [0][0].isSuccess()) \{ 
Observation.java

291

292

293

294

295

296 callback");

true) \{
syncResult = "success";

Log.i("edits", syncResult);

Intent intent $=$ getIntent () ;

Log.i("edits", syncResult);

if (syncResult $==$ "success") \{

\}

setResult(RESULT_OK);

if (filePath $!="$ ") \{

int obj $=$ editResult $[0][0] \cdot \operatorname{getObjectId}()$;

File fileP = new File(filePath);

featureLayer . addAttachment(

obj,

filep,

CallbackListener <FeatureEditResult $>()\{$

new public void onCallback( FeatureEditResult objs)

// TODO Auto-generated

// method stub

if (objs.isSuccess $($ ) $==$

"pictureSuccess";

297

298

299

300

301

302

303

304

305

306

307

308

309

310

311

312

313

314

315
syncResult $=$

\}

Log.i("edits", "attachment

\}

public void onError( Throwable e) \{

// method stub

Log.i("edits", "attachment error"); Log. i("edits", e.getMessage( ));
// TODO Auto-generated

\}

\}); 
Observation.java

316

317

318

319

320

321

322

323

324

325

326

327

328

329

330

331

332

333

334

335

336

337

338

339

340

341

342

343

344

345

346

347

348

349

350

351

352

353

354

355

356

357

358

359

360

361

362

363

364

365

366

367

368

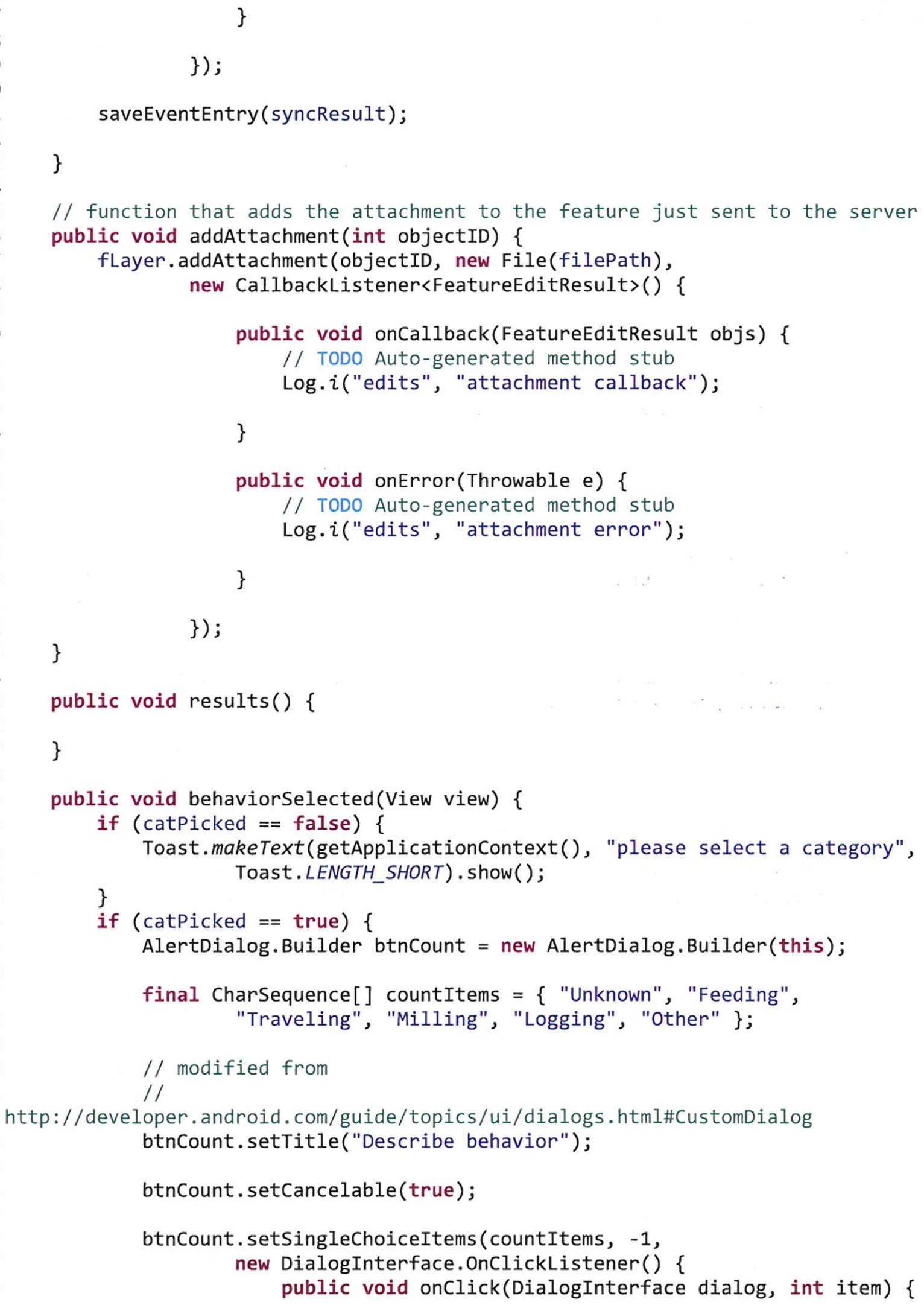




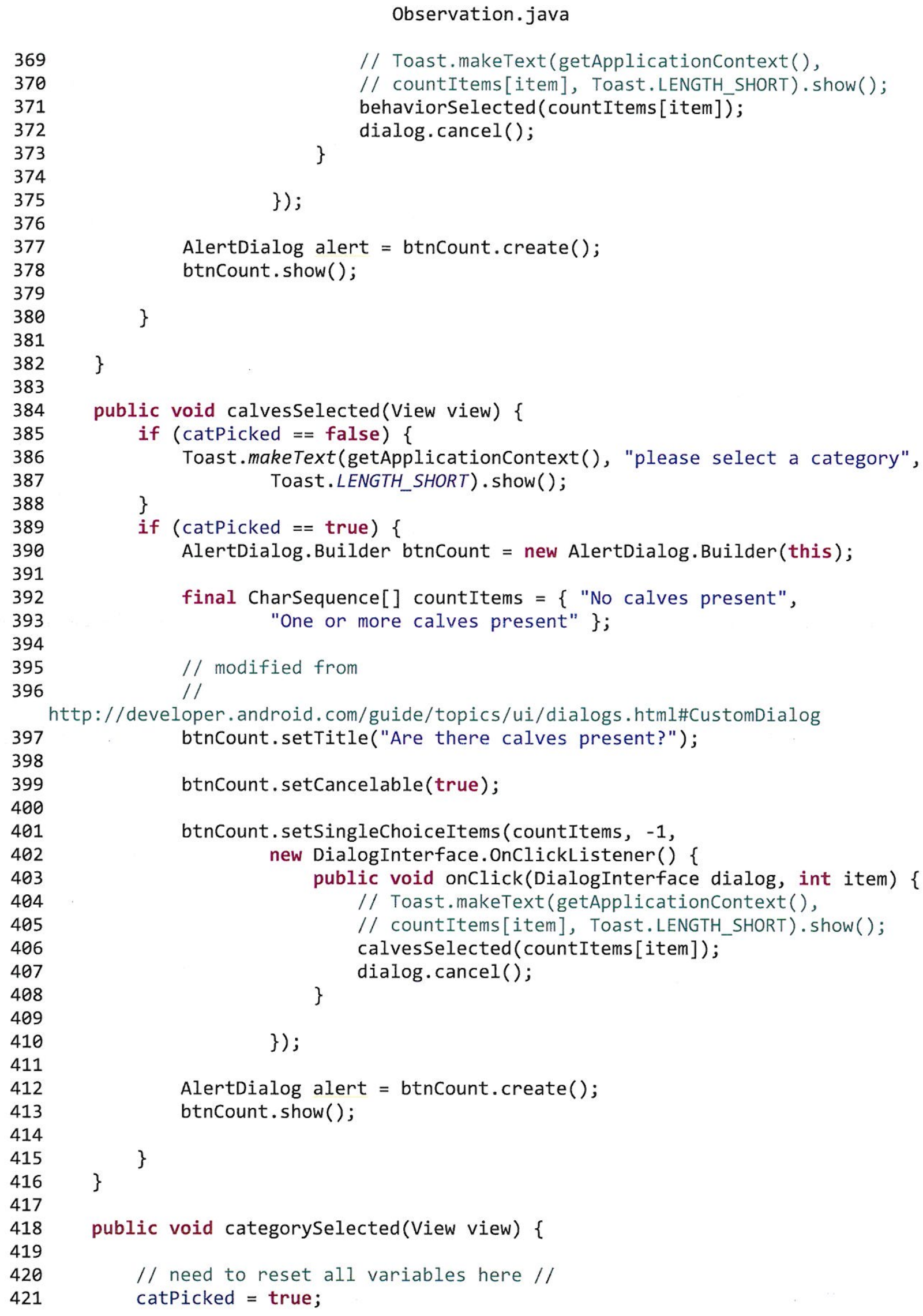

Observation.java 
Observation.java

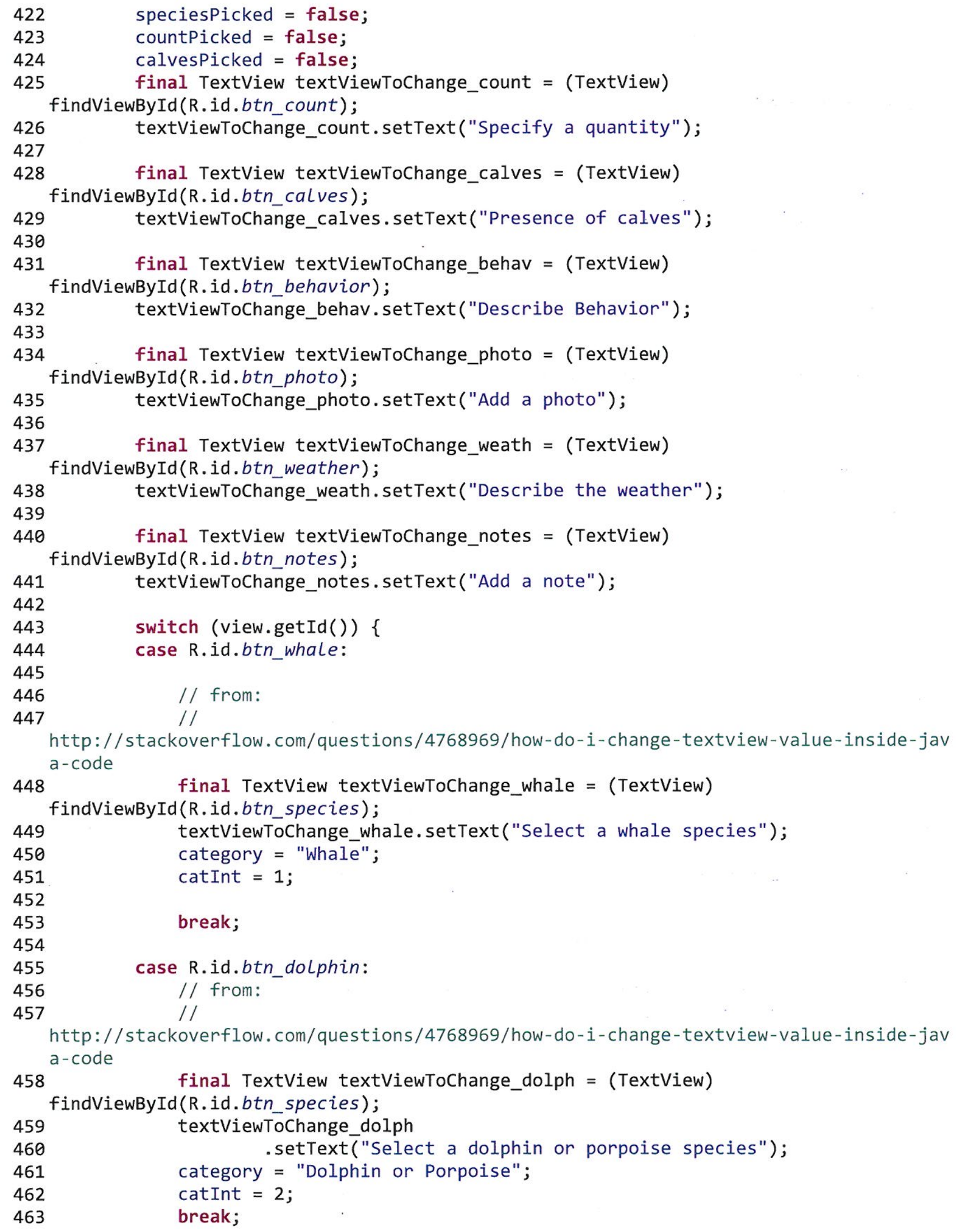


Observation.java

464

465

466

467

http://stackoverflow.com/questions/4768969/how-do-i-change-textview-value-inside-jav a-code

468 findViewById(R.id.btn_species);

469

470

471

472

473

474

475

476

http://stackoverflow.com/questions/4768969/how-do-i-change-textview-value-inside-jav a-code

case R.id.btn_unknown:

// from:

//

case R.id.btn_seal:

// from:

/I

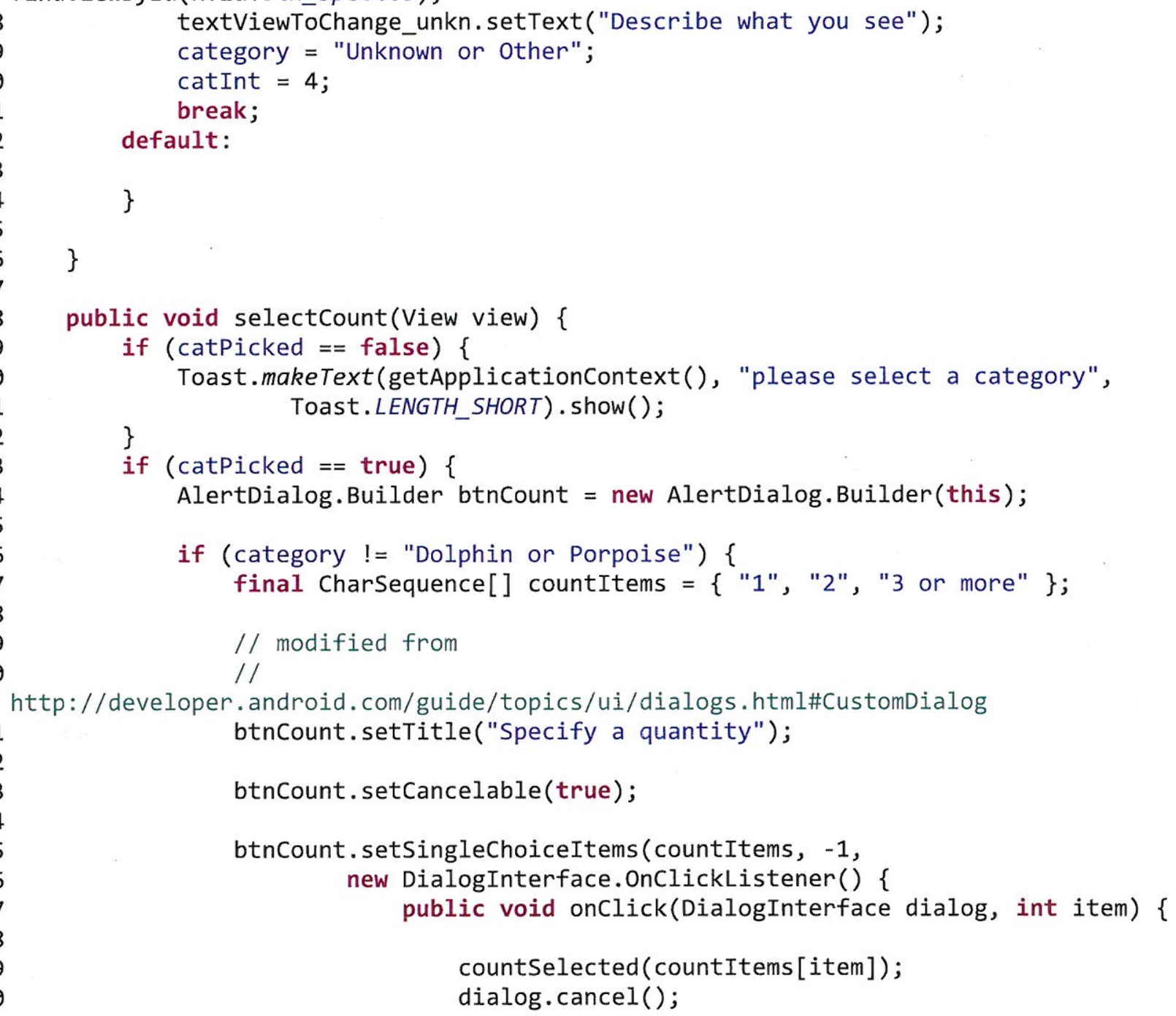


Observation.java

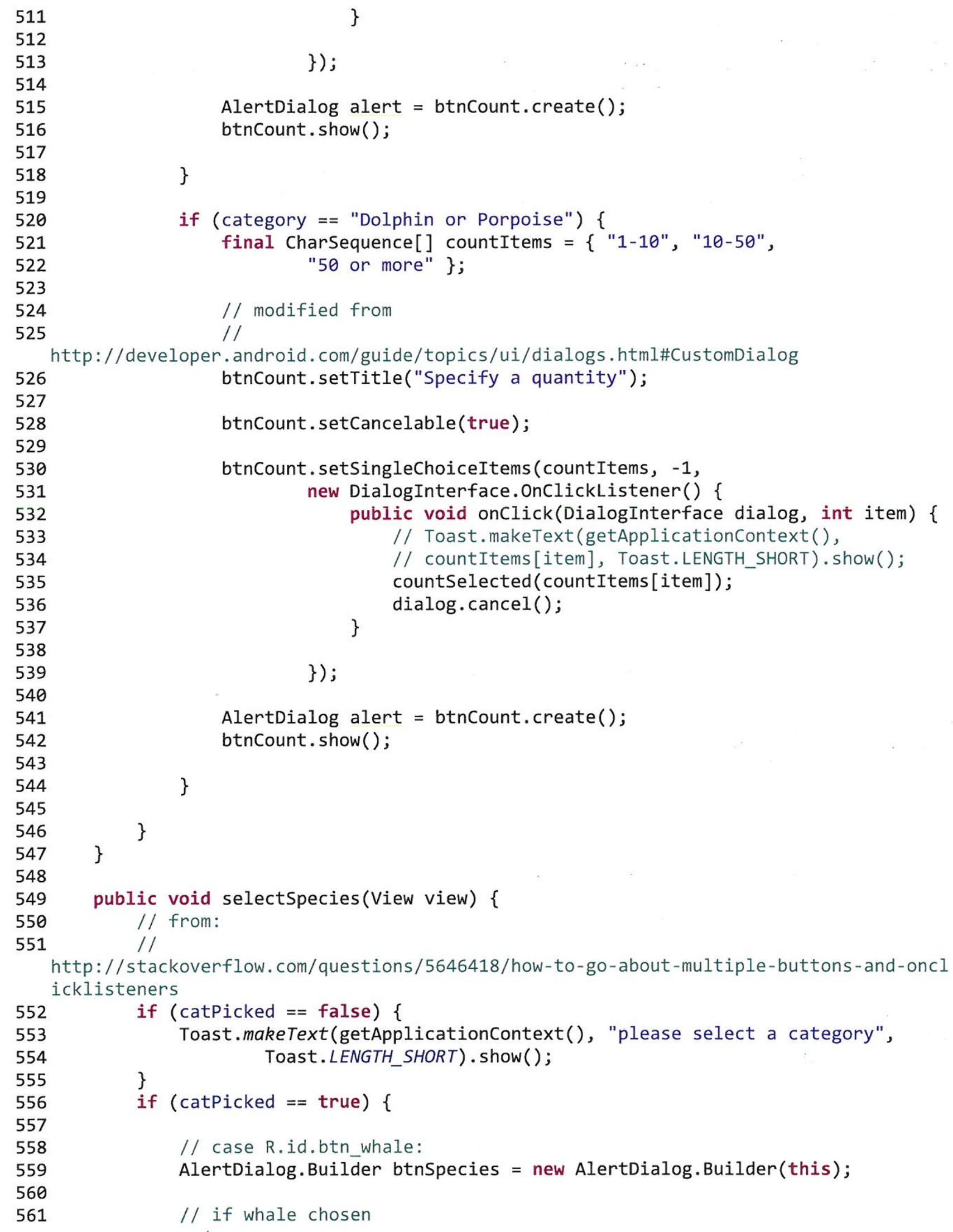


Observation.java

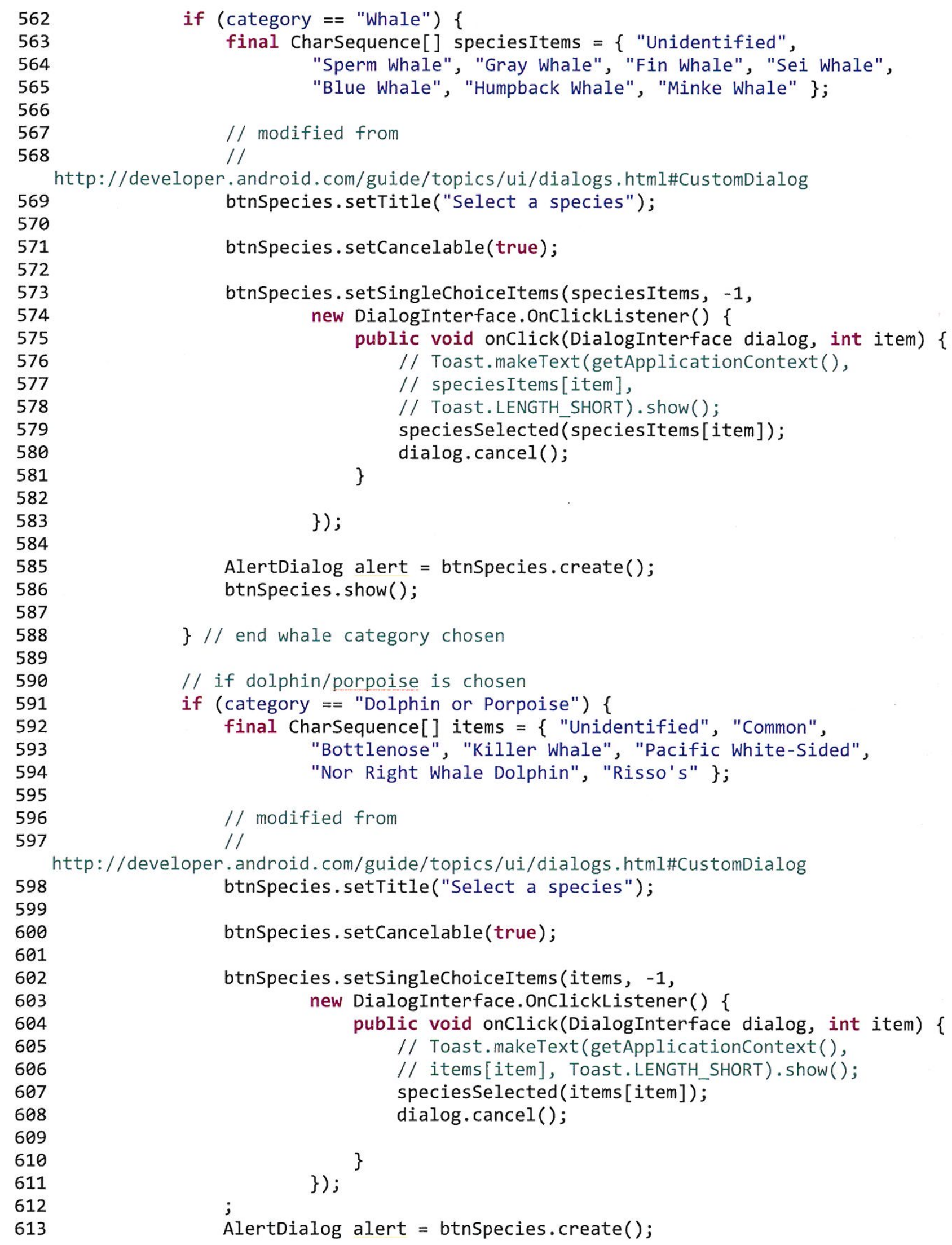

AlertDialog alert $=$ btnSpecies. $\operatorname{create}()$; btnSpecies. show();

\} // end whale category chosen

// if dolphin/porpoise is chosen

if (category $==$ "Dolphin or Porpoise") \{

final CharSequence[] items $=\{$ "Unidentified", "Common", "Bottlenose", "Killer Whale", "Pacific White-Sided", 
Observation.java

614

615

616

617

618

619

620

621

622

623

624

625

626

627

628

629

630

631

632

633

634

635

636

637

638

639

640

641

642

643

644

645

646

647

648

649

650

651

652

653

654

655

656

657

658

659

660

661

662

663

664

665

666

btnSpecies.show();

\} // end dolphin/porpoise category chosen

// if seal/sea lion is chosen

if (category $==$ "Seal or Sea Lion") \{

final CharSequence[] items $=\{$ "Unidentified", "CA Sea Lion", "Harbor Seal", "N. Fur Seal" \};

// modified from

//

http://developer. android.com/guide/topics/ui/dialogs.html\#CustomDialog

btnSpecies.setTitle("Select a species");

btnSpecies.setCancelable(true);

btnSpecies.setSingleChoiceItems (items, -1, new DialogInterface.OnClickListener() \{ public void onClick(DialogInterface dialog, int item) \{

// Toast.makeText(getApplicationContext(),

// items[item], Toast.LENGTH_SHORT).show(); speciesselected(items [item]); dialog.cancel();

\}

\});

;

AlertDialog alert = btnSpecies.create();

btnSpecies.show();

\} // end seal/sea lion category chosen

// if unknown is chosen

if (category $==$ "Unknown or Other") \{

AlertDialog.Builder alert $=$ new AlertDialog.Builder(this);

alert.setTitle("What do you see?");

// Set an EditText view to get user input

final EditText input = new EditText(this);

alert.setView(input);

alert.setPositiveButton("Ok", new DialogInterface.OnClickListener() \{

public void onclick(DialogInterface dialog, int whichButton) \{

Editable value = input.getText ();

speciesSelected(value);

\}); 
Observation.java

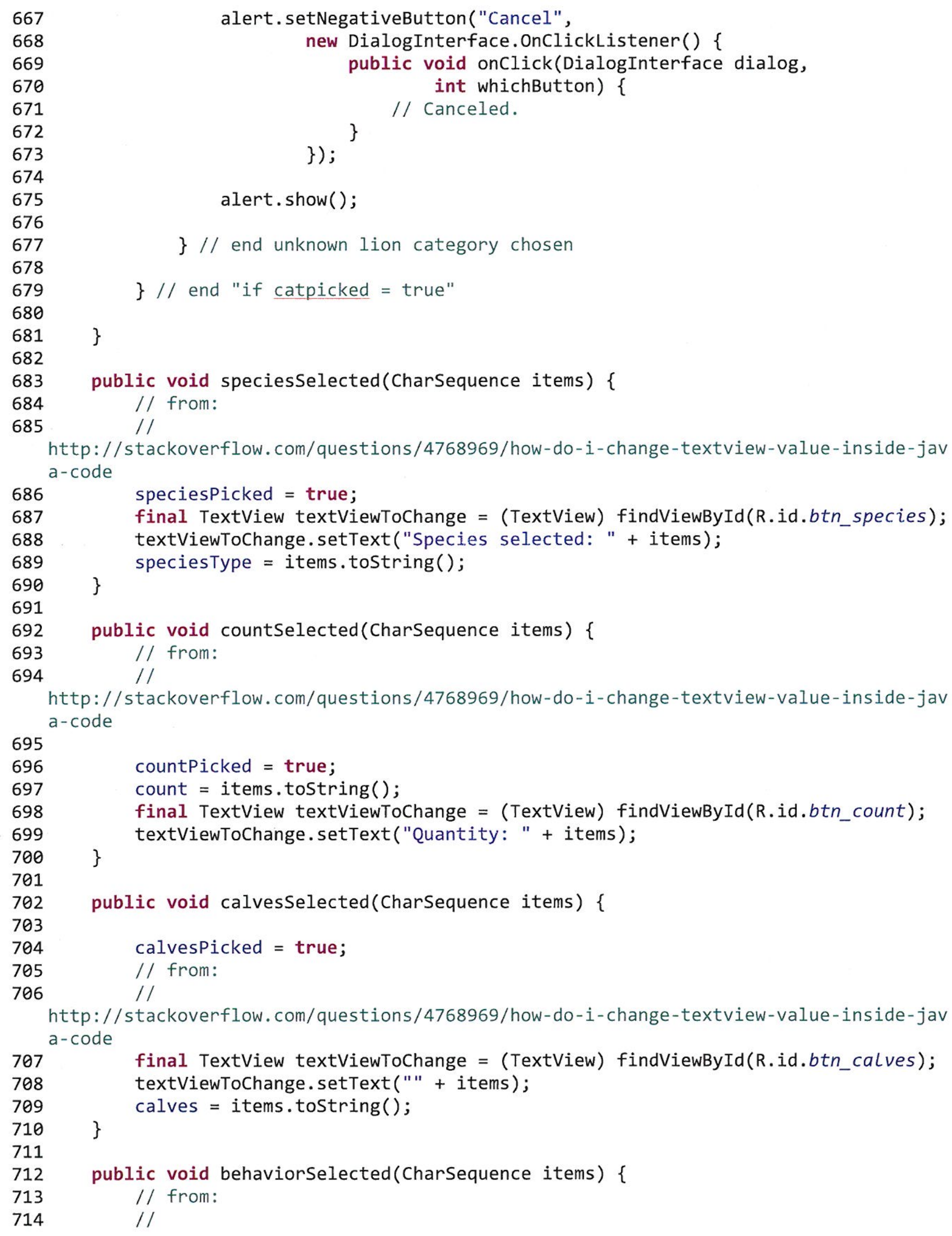

667

668

669

670

671

672

673

674

675

676

677

678

679

680

681

682

683

684

685

http://stackoverflow.com/questions/4768969/how-do-i-change-textview-value-inside-jav a-code

686

687

688

689

690

691

692

693

694

http://stackoverflow.com/questions/4768969/how-do-i-change-textview-value-inside-jav a-code

695

696

697

698

699

700

701

702

703

704

705

706

http://stackoverflow.com/questions/4768969/how-do-i-change-textview-value-inside-jav a-code

707

708

709

710

711

712

713

714

alert.setNegativeButton("Cancel", new DialogInterface.OnClickListener() \{ public void onClick(DialogInterface dialog, int whichButton) \{

\});

// Canceled. \}

alert.show( );

\} // end unknown lion category chosen

\} $/ /$ end "if catpicked = true"

\section{\}}

speciesPicked = true;

final TextView textViewToChange = (TextView) findViewById(R.id.btn_species); textViewToChange.setText("Species selected: " + items);

\} speciesType = items.toString ();

public void countSelected(CharSequence items) \{

// from:

$/ /$

countPicked = true;

count = items.tostring () ;

final TextView textViewToChange $=$ (TextView) findViewById(R.id.btn_count);

\}

textViewToChange.setText("Quantity: " + items);

public void calvesSelected(CharSequence items) \{

calvesPicked = true;

// from:

// 
Observation.java

http://stackoverflow.com/questions/4768969/how-do-i-change-textview-value-inside-jav a-code

715 final TextView textViewToChange $=$ (TextView)

findViewById(R.id.btn_behavior);

http://stackoverflow.com/questions/4768969/how-do-i-change-textview-value-inside-jav a-code

http://stackoverflow.com/questions/4768969/how-do-i-change-textview-value-inside-jav a-code

http://stackoverflow.com/questions/4768969/how-do-i-change-textview-value-inside-jav a-code

final TextView textViewToChange = (TextView) findViewById(R.id.btn_photo); textViewToChange.setText("Path: " + items);

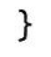


Observation.java

public void onClick(DialogInterface dialog, int whichButton) \{ Editable value = input.getText (); noteAdded(value);

$/ /$ Toast toast $=$

// Toast.makeText(getApplicationContext(), value,

// 5000);

// toast.show(); \});

alert.setNegativeButton("Cancel", new DialogInterface.OnClickListener() \{ public void onclick(DialogInterface dialog, int whichButton) \{

\}); // Canceled. \}

alert.show (); \}

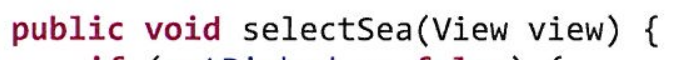




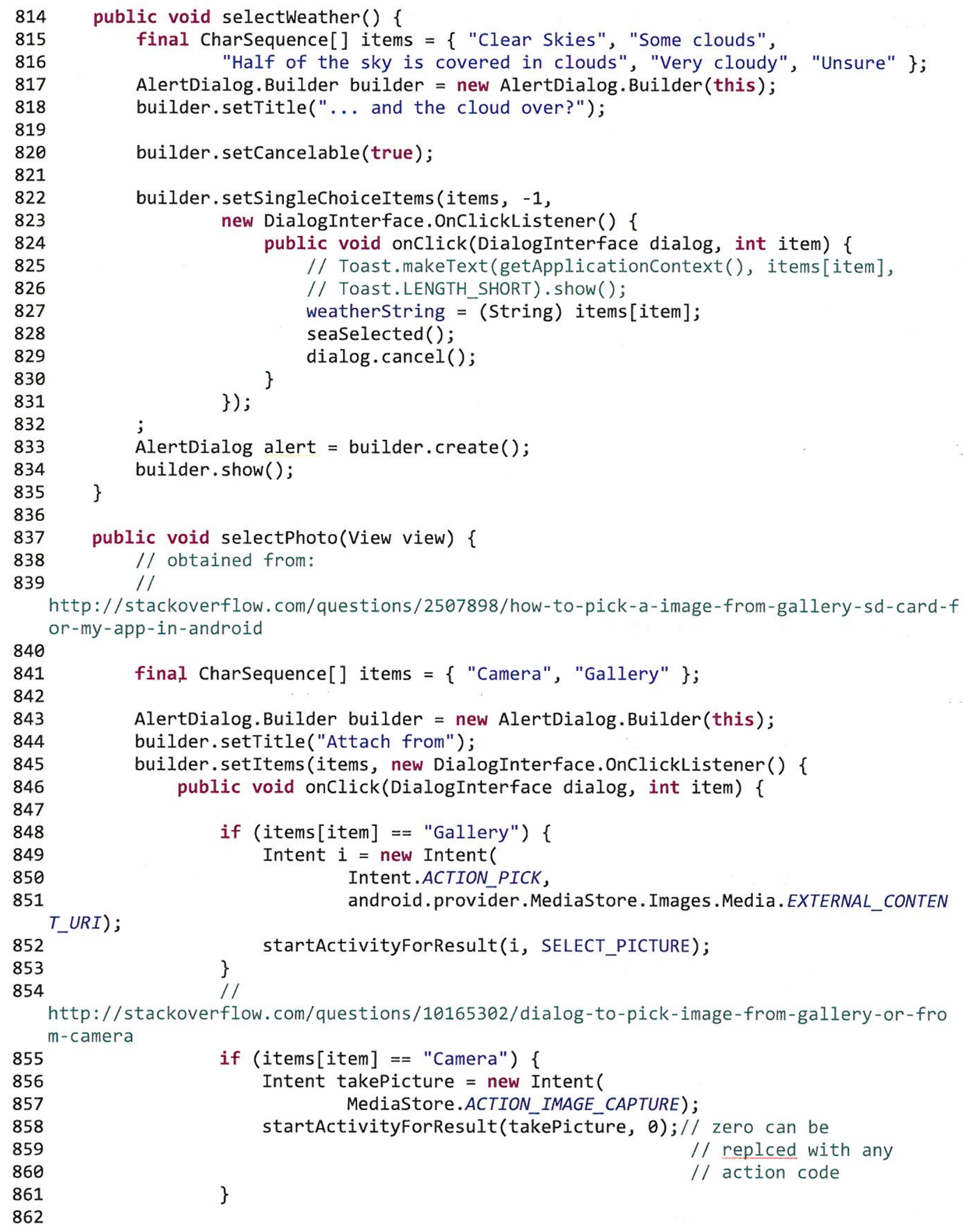


Observation.java

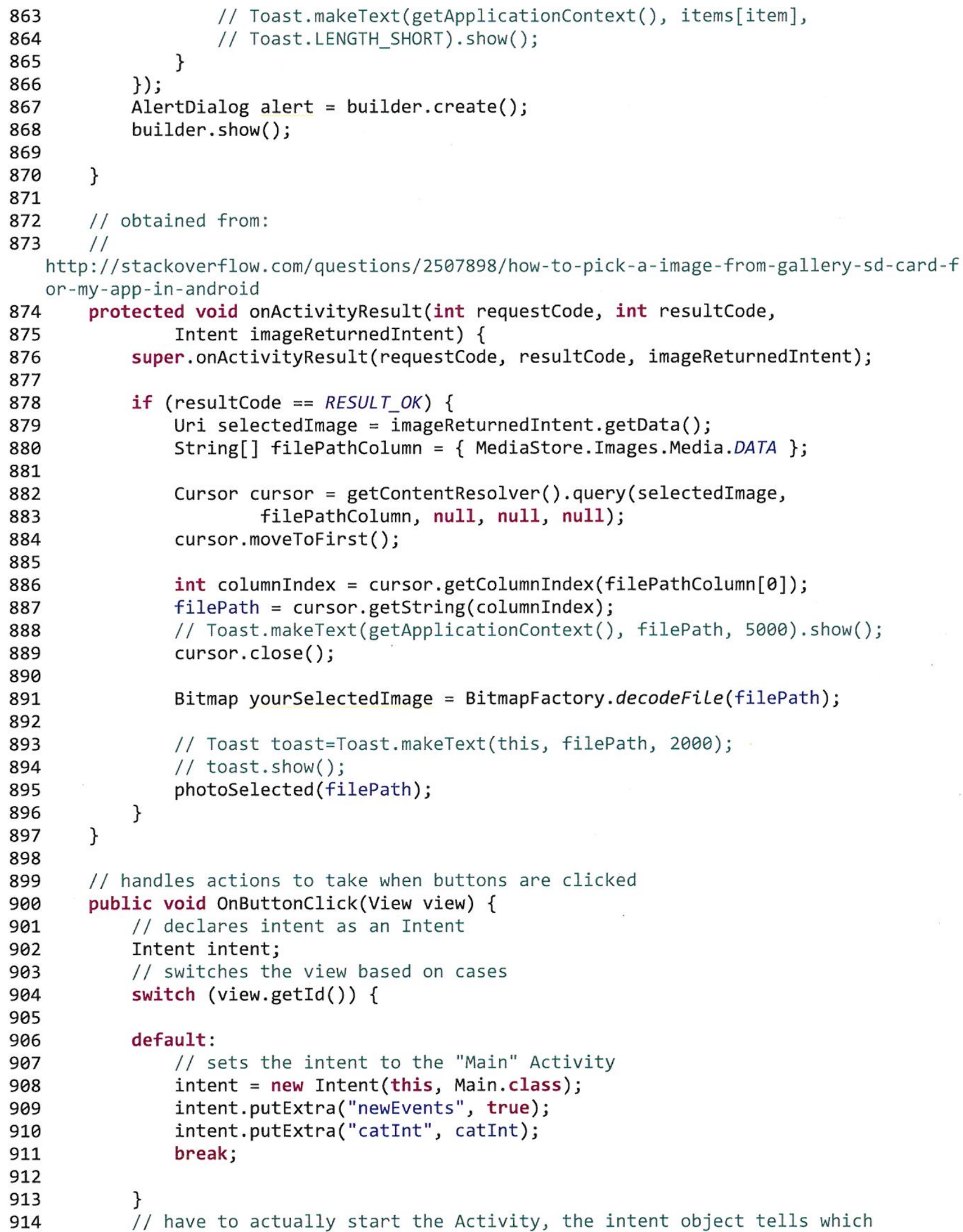


Observation.java

915

916

917

918

919

$920\}$

921

$922\}$

923 

Main.java

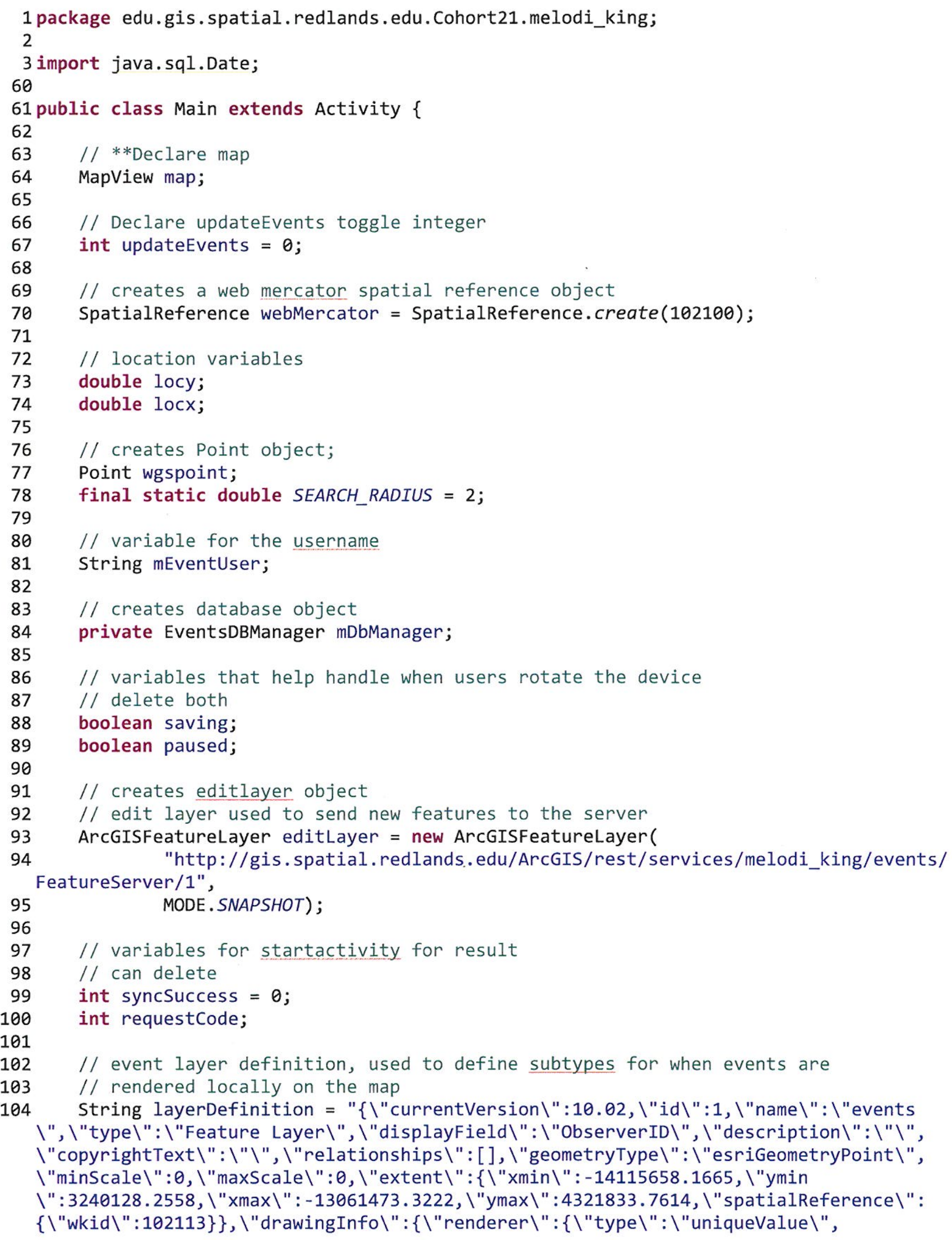


Main.java

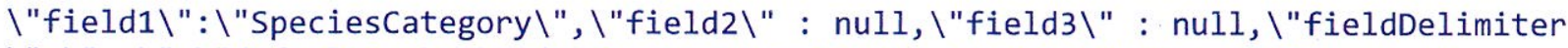

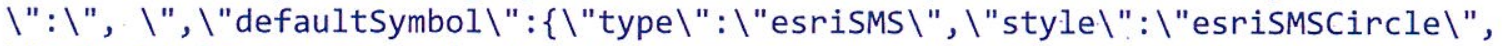
$\backslash$ "color $\backslash ":[0,158,37,255], \backslash "$ size $\backslash ": 4, \backslash$ "angle $\backslash ": 0, \backslash$ "xoffset $\backslash ": 0, \backslash$ "yoffset $\backslash ": 0$,

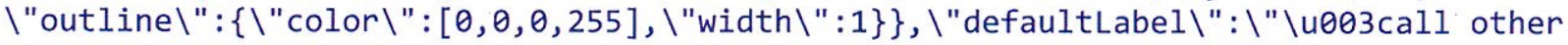
values $\backslash$ ue03e $\backslash ", \backslash$ "uniqueValueInfos $\backslash ":[\{\backslash$ "value $\backslash ": \backslash " 2 \backslash ", \backslash "$ label\": \"Dolphin or

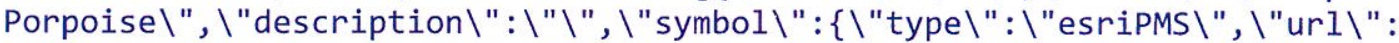
$\backslash "$ B698AF73\",\"imageData \":\"iVBORwOKGgoAAAANSUhEUgAAABgAAAAZCAYAAAArk +5dAAAAAXNSROIB2cksfWAAAAlwSFlzAAAOXAAADsQB1S sOGWAAAwFJREFUSInllwtIk2EUx397t/mmstSZS 20i3iorDbbuYhQlkUFGYGFEd6w+FEVpRZFhSR +EUAlBsQhaQh/EriSRYjUUwyS3LphamJdZ5BtUZi/uOpcarc1pQRBOPj1wzvP/PeccznlU/GVT/RcAtZE1ux NISQsiJFSFSpAZ/tSNtbeJmnbABNj/CJBN/towUVe4UF496zEN6rksJZQIt38bBc011C5rFE0Vsiw3/xZg13 j2mF6edSxZnq/po4ty91HITQ9ACFMW7+D0giR5XtB59oYDtyYEyOPi01GeXJDMfBHAzDUASjj IevbgYBSAqe hJxKhMJyv7K1+o4lA38GIcgEsxQnmFkVUiwHsGqOUcABJdVHHYI1pLLPspI40crA6ahh5Ss9cvoFC8kR8kz9 E5sNNCHSaKfSXpNokeTpFFEXcCVrJ1ZldUU6rNZrOMCfggS1uSMHCPai5wXEtQSyKbOcx0jIShQ2aEFuqopp g8KhZim5WJfgCBTIoRUFJLiZd4NvlkspMgND +VQI2RFVRWgGc0BxpYob9Nx9glUqAMduJEWUYFSCDFQ/yHWTBzlCuEEEEWYcE/ +3w12fGeAWEG6Wwij1hmEkAgfbykHhORxBNFnDvaiZPpGJhKDACt3PMYOi+Ag9GhYT5GHqXK47WXJLOdMzh + GVoBWS3UXMUQfYN +ARFafYNLYPOvUihQoELtnfR366ez36Ktq0HyA4iWkk484s5GPUnKH6IALlzus51RnyArD9okSXriN4Mcpro uӨZovWSXzTjUiGrToSaSdRvTMYArRWHIIFPHoiKGeqXhYzjt637TTePXXPZ

+rYraUltvE7blveTVPSyQdtJFAKiN8wYqZSOKwY8eKmcWsoY36z328LHpCg3VCgAwUzkqeLVIimLp5uiGFJY KGUDSEo/veUIBworFwV30QziPXKav1pTXmNs1ltgPIqaTtdAetpeBMDSBQC4LgwV5XmOEeSRy8e1k +cRL40pbOuB90LobnQIZPpzze7X/ky/zPAd8AJIX1ZOTUIUWAAAAASUVORK5CYII= \", \"contentType $\backslash$ ":

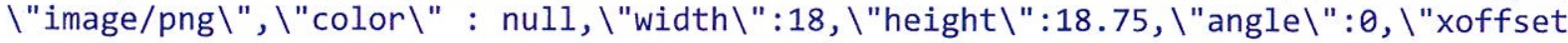

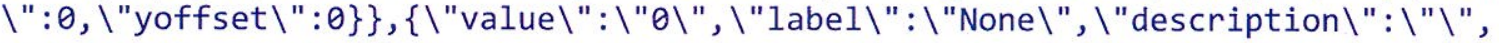

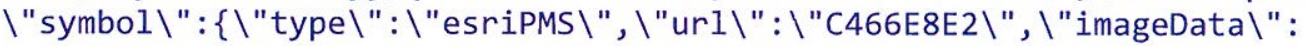
\"iVBORWOKGgOAAAANSUhEUgAAAAWAAAAMCAYAAABWdVZnAAAAAXNSROIB2cks FWAAAAIWSFIzAAAOXAAADs QB1Ss0GWAAAJ1JREFUKJGtObEKQVEYB/DFQDezDBZ5C6U8AQm7V7CIyWSUhzGZWL2EZAaZ9WWxUPderlLOeM 75nf7/75T8uEr/AHWs0Hue7zDH8R0o4oBmam

+ADlo4ZUCSJIUISF90P7TGMAMiYvQlfheVfKT6F1BGLQ/OaBSAOy75DpuImBSALW7.5Dkv0ZacEV0x5H +sVbdl/2GOGOyfw6jEuiOUB/ZOhYNqEvhSAAAAASUVORK5CYII= \", \"contentType \": \"image/png \",

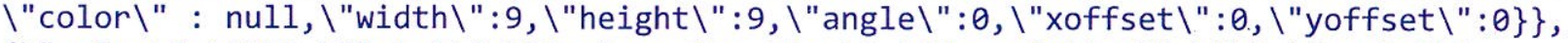

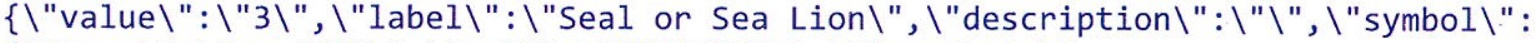

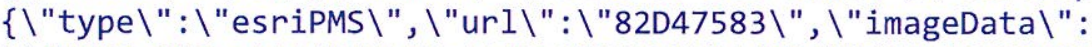

\"iVBORwOKGgoAAAANSUhEUgAAABKAAAAZCAYAAADE6YVjAAAAAXNSROIB2cks fwAAAAIwSFlzAAAOXAAADs QB1SSOGWAAAXVJREFUSInt1V1MU2cYwPF/vziFFkIOiJRaCREiH5HVr3WgQUKyGDUabOS3i4Vkxl0Yw41ZRm K2+HG9hMwtMdkFRi8gJkuWLCXzi1kmk4ABK4qiVJqWWaEIr9oK9NDT010QiaW20C8WE32uznne9zy/5zk5H2 b+hzC/U4ilqVT+oswp7cgzZeVjwajGFi0jQTH+eOC9DVwGtDdGWjw1B9bYtDMNG4uqc7MtlqTF7XD8ebT3Yr + /sfu +uKCqau9/Rlqbyts2FFjaal1rc9Ptybdb61qbqj7c5AjmnPnVVwD88trIuf01B21Wvql1yVI6ILwQpW9smpF /hCmscqi1sdTc/mfgEXB/VSQBhq817cI0lzMtAHCl38+1AQWArqP1F0VZ940oEeW3B

+L4qsj5I54vyyWKDBmA6GJsGQAotGVhgKwDm9dXDYWIWkVRhjIiT

+ZmP6t2rM80BHcnRPI1C4sU2q1UOmQPwrsXyIXIRpPL1GYMLa4TEBG

$+6 /$ Y15aeezlNot2IykLOrXF7XOZzCRApiNpptL5/H4zpBEeHW

+AzdQ1P4hJqCz8diy8c2u2RPqbkyoaPFAWMCGJ0SdPYHueYTK7etaOylptSs1BczBdF0ZvUExX1jIdp +Hs5Y/EU48paa1X0gPJ8KrYqU5NuvTTyZ

+9T/OLn7UgnqqmSskkRH39KTJQFuh8SCppEAAjPPJm8K2xVIvqUpSF1BzqmeUXG4vqLENPtMZVGDbDPs2VKG S871Ys8DAFo8Dpq31TMYnObGWO10HW4FZ7XCCO

+qkzR8e91/qcXT8XRe/fzEbnfS201HIdSoyvfNbqqdMgYD7KXWsrPCyWAgNP63L/DVynqvRACKO/qOhY +4PxiZFNsqS+Tl/0Z1MvUb1qbsvzcpRP

+Ictqrc0e1kY9BH27yfvTXH1sv94yGmt0u2Wi3WsiWkj/Ec9EYvf5pf2A6fLJzWPzOqlppEYCaY8Rh4J0uo1 vP3plQ2h06qdZiRjZiNmo6kbmoFpyZf3z1h

+viFBBNVycj8iI0/zhwb2m4N4+35vf7HlmOfwEYGSjqIxnPkQAAAABJRU5ErkJggg== \", \"contentType

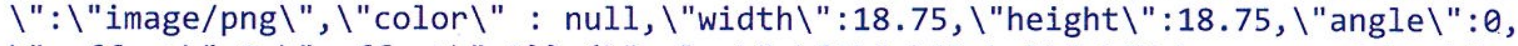

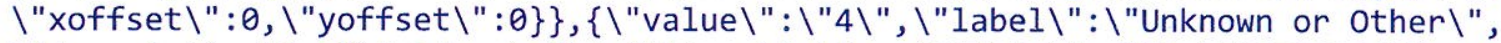

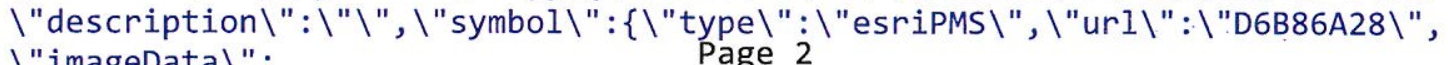
$\backslash$ "imageData $\backslash$ : 
Main.java

105

106

107

108

109

110

111

112

113

114

115

116

117

118

119

120

121

122

123

124

125

126

127

128

129

130

131

132

133

134

135

136

137

138

139

140

141

142

143

144

145

146

147

148

149

150

151

152

153

154

155

156

// creates featurelayer object

ArcGISFeatureLayer fLayer;

// creates cursor object

public Cursor mCursor;

// interval for handler

// private int m_interval $=300000$; // 5 min by default, can be changed

// later

private int $\mathrm{m} \_$interval $=30000$;

// creates handler object for repeating task

private Handler m_handler;

/** Called when the activity is first created. */

//@Override

public void oncreate(Bundle savedInstancestate) \{

super. oncreate( savedInstancestate);

setContentView(R. layout.main);

//**Gets user ID from Home event

mEventUser $=$ getIntent ()$\cdot$ getStringExtra ("user");

// creates new map view from viewID

map $=($ MapView) findViewById(R.id.map);

// initiates database object

mDbManager = new EventsDBManager(this);

// adds the basemap

// map.addLayer(new ArcGISDynamicMapServiceLayer( ap/MapServer"));

"http://services.arcgisonline.com/ArcGIS/rest/services/World_Street_M

map. addLayer(new ArcGISDynamicMapServiceLayer( MapServer"));

"http://services.arcgisonline.com/ArcGIS/rest/services/Ocean_Basemap/

// editLayer isn't drawn, it is just used for sending position updates

// to the server

editLayer.setVisible(false);

map.addLayer(editLayer);

// calls the updatevents layer function

updateEventsLayers();

//***Code snippet modified from Esri's Nearby Sample project

map. setOnStatusChangedListener(new OnStatusChangedListener() \{

private static final long serialversionUID $=1 \mathrm{~L}$;

// function that handles location changes, I got this function

Page 3 
Main.java

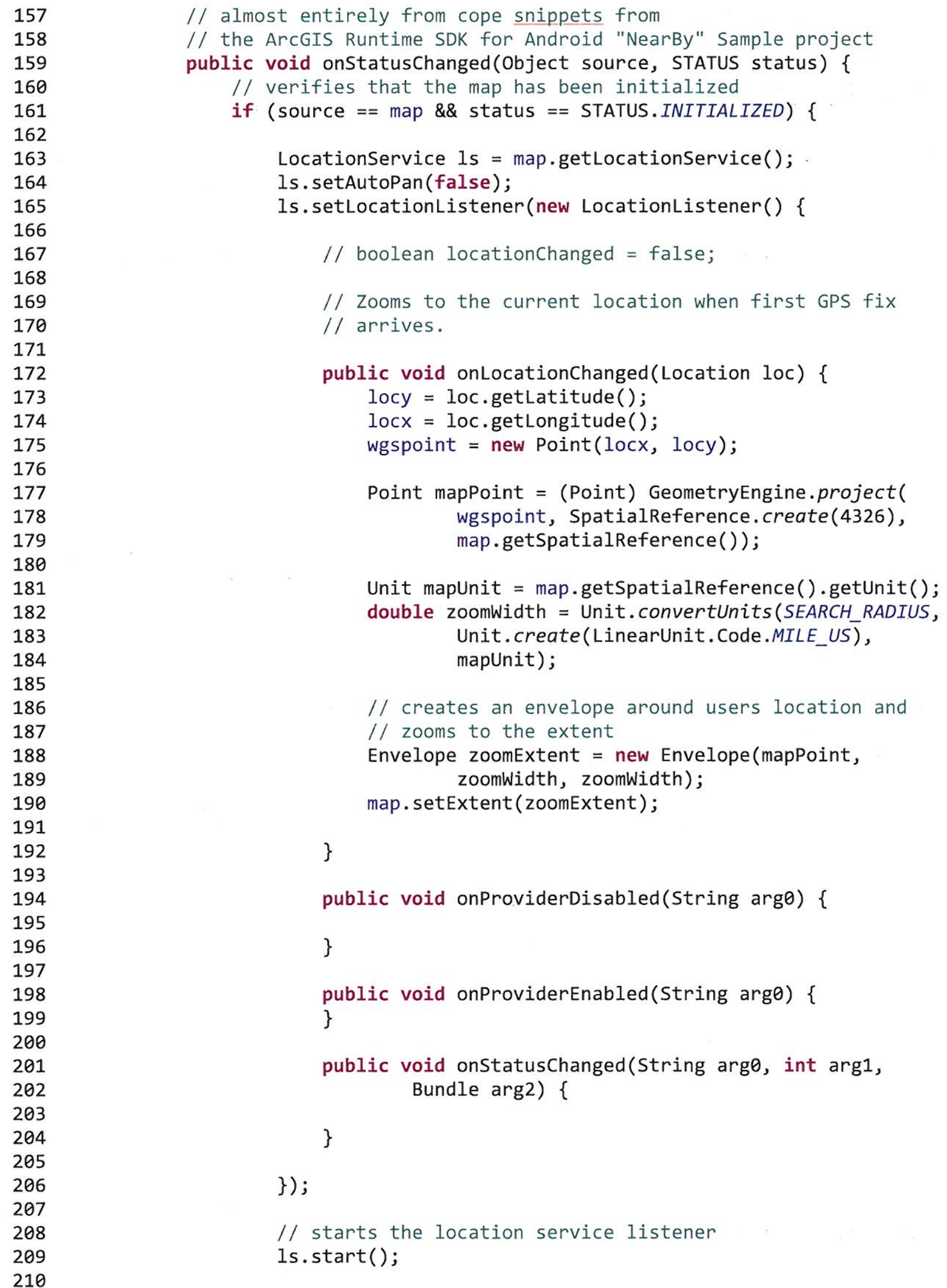


Main.java

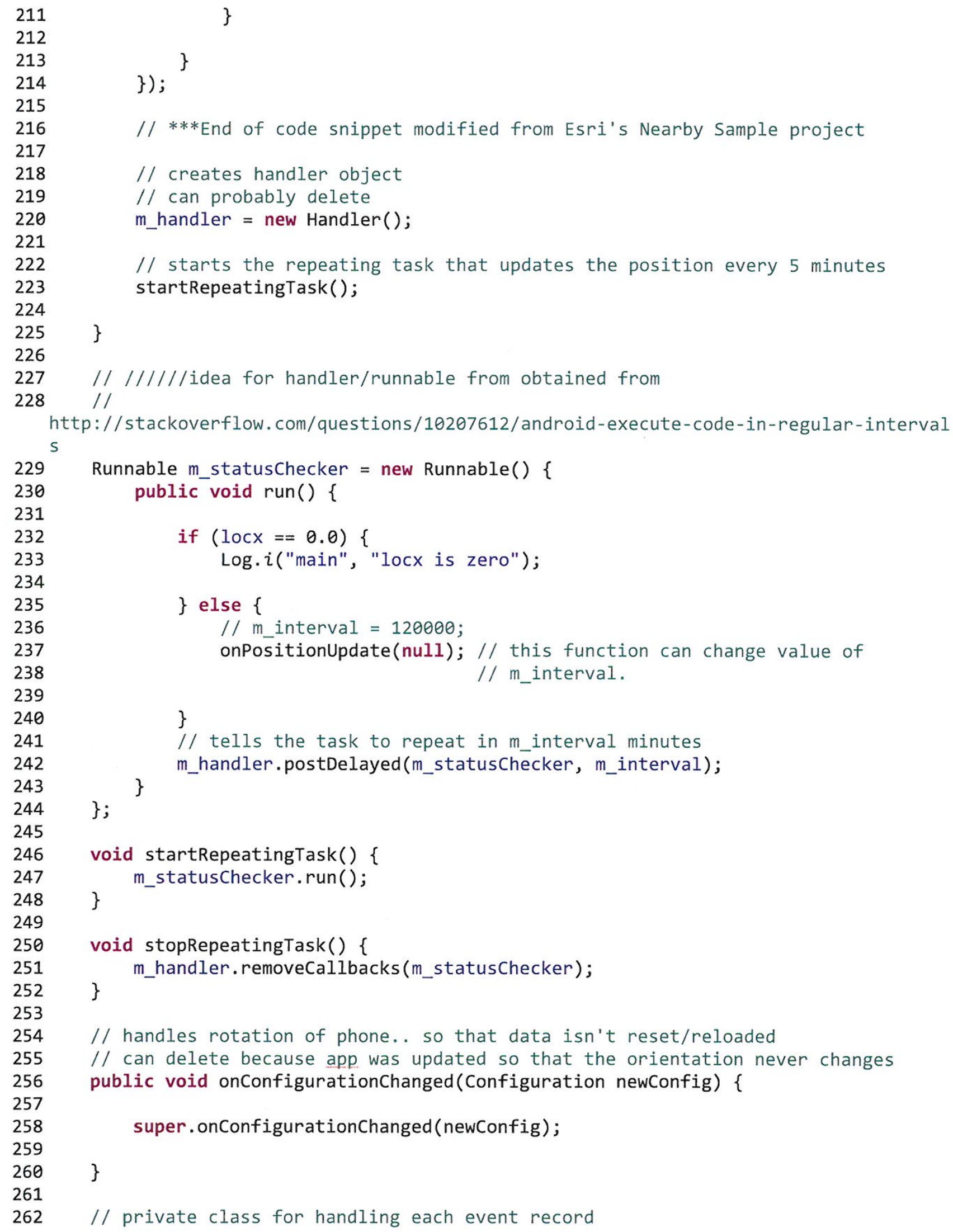


Main.java

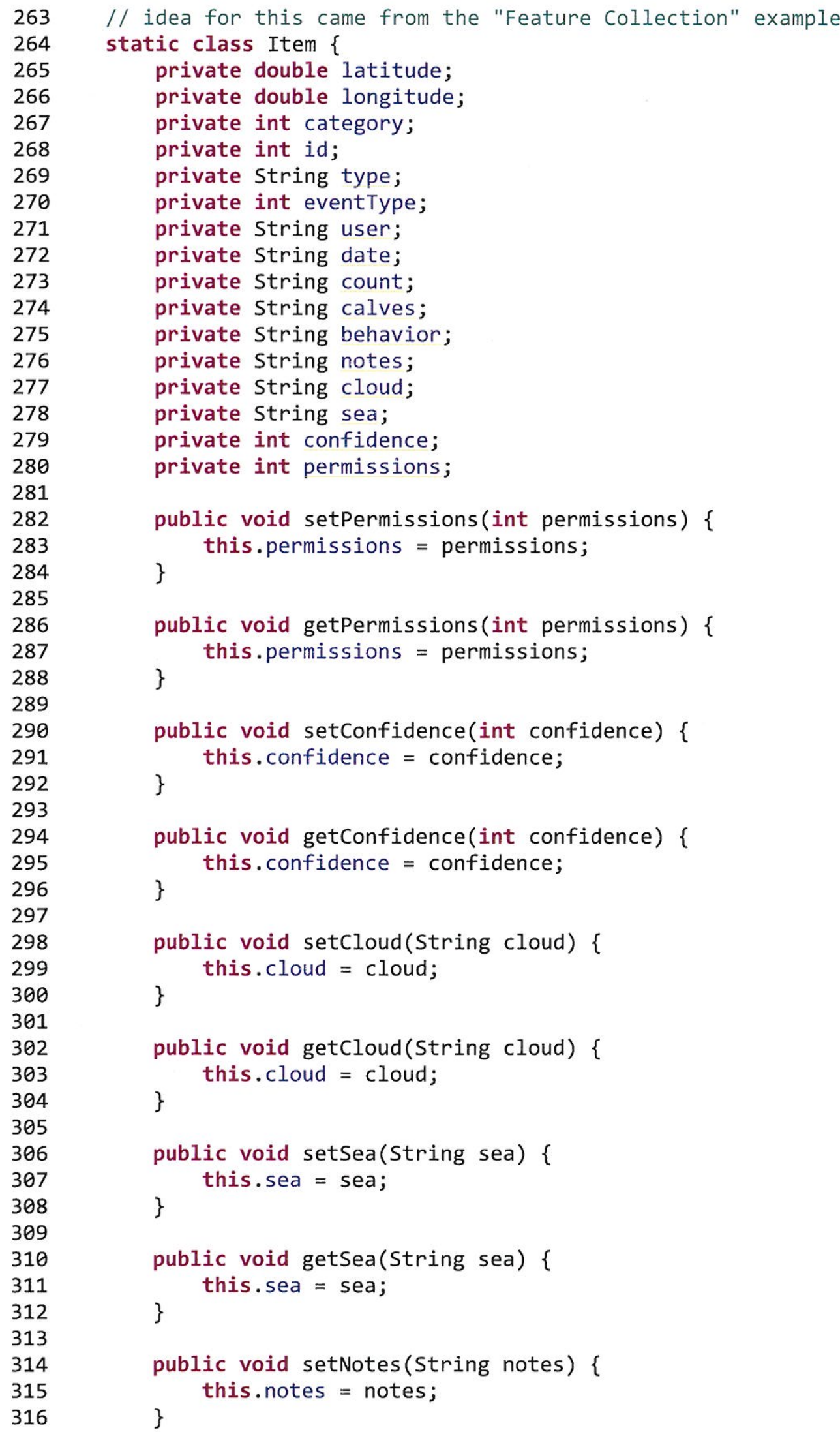




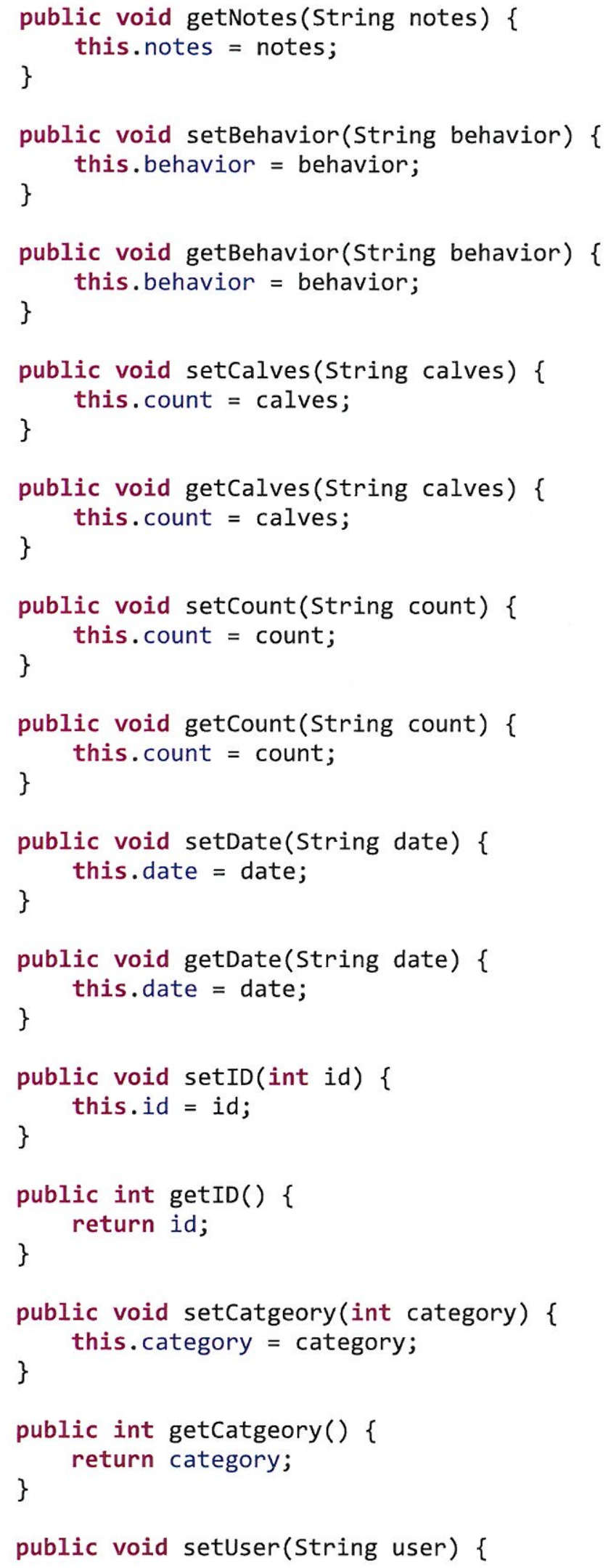


Main.java

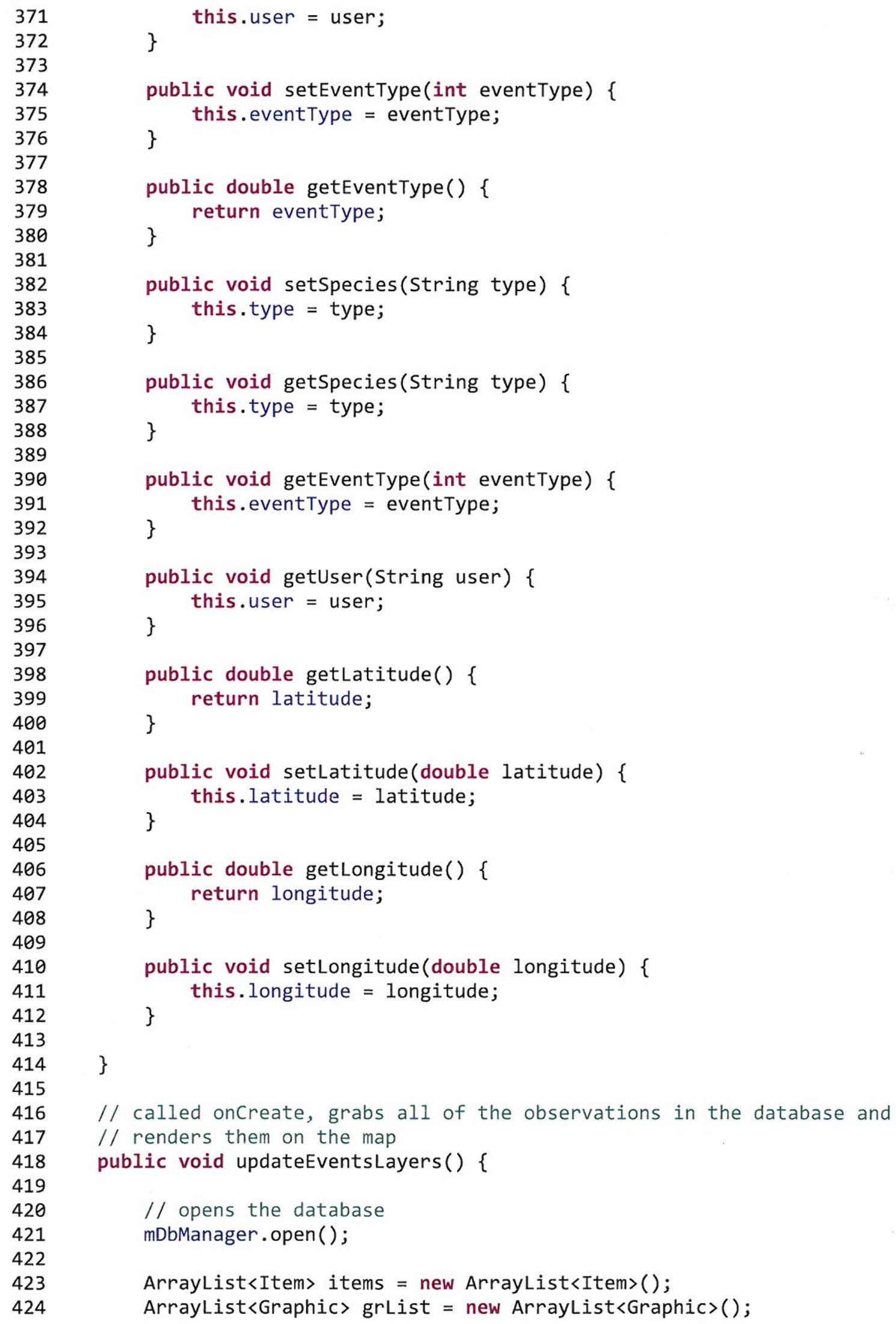


Main.java

425

426

427

428

429

430

431

432

433

434

435

436

437

438

439

440

441

442

443

444

445

446

447

448

449

450

451

452

453

454

455

456

457

458

459

460

461

462

463

464

465

466

467

468

469

470

471

472

473

474

475

476

477

478

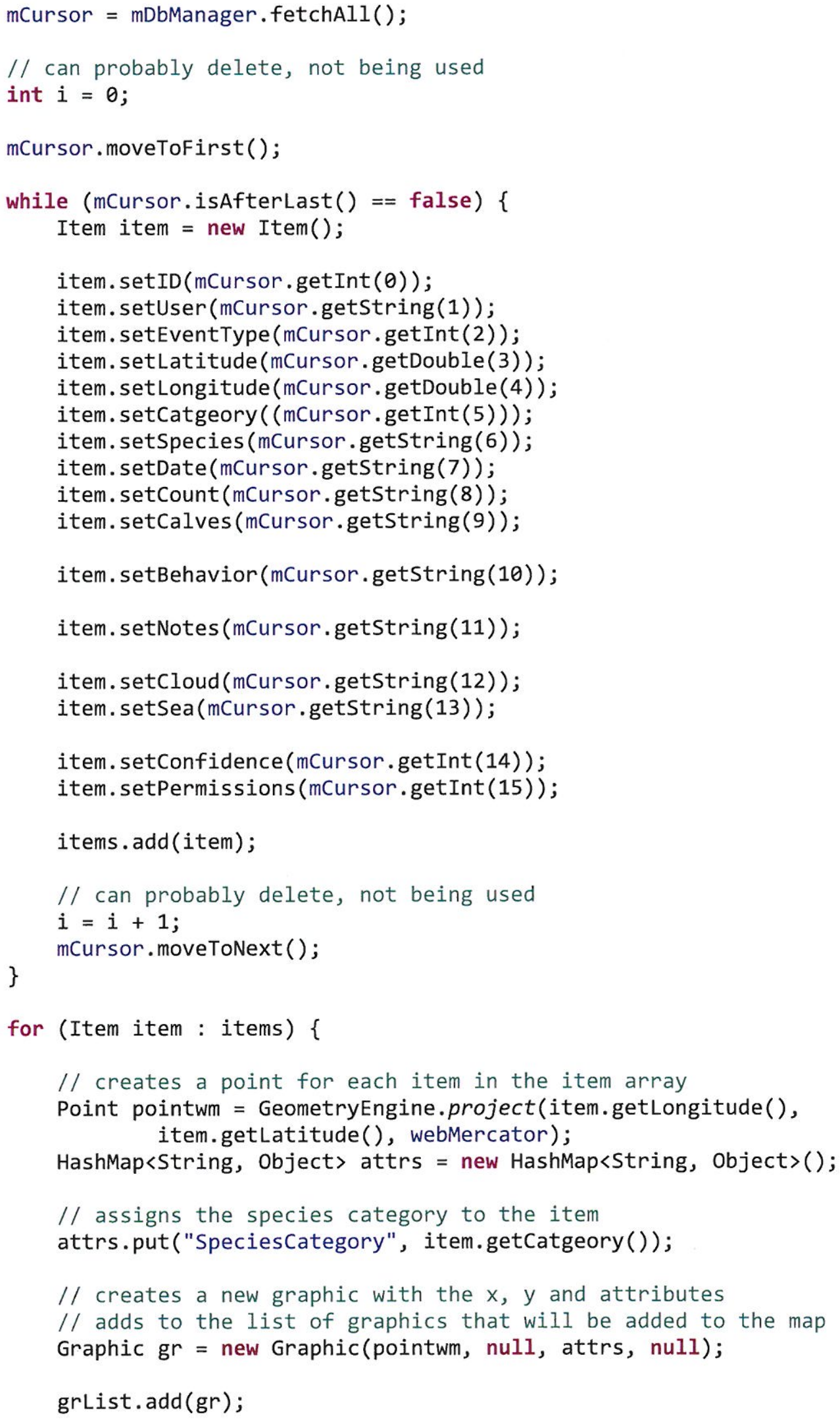


Main.java

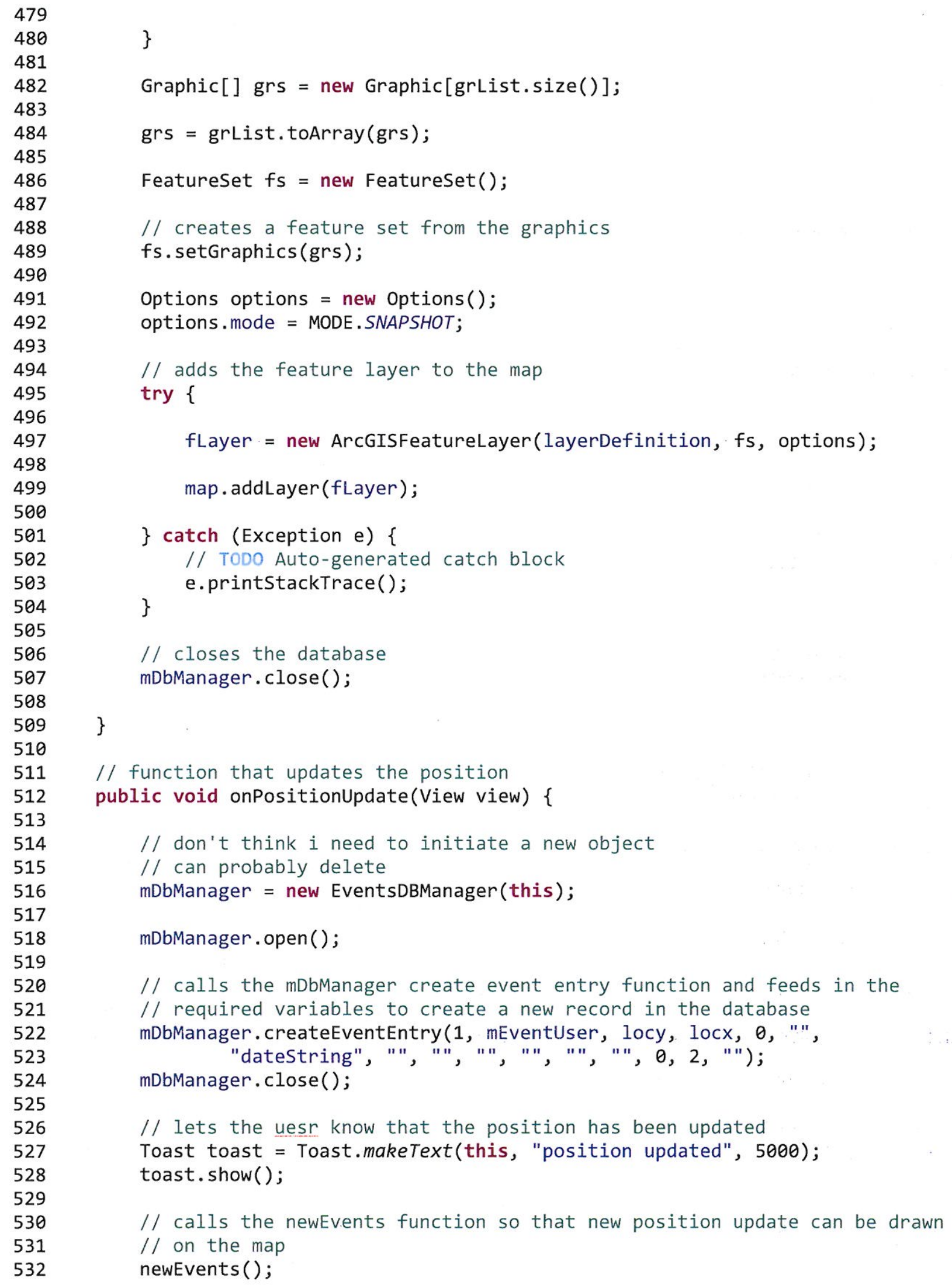


Main.java

533

534

535

536

537

538

539

540

541

542

543

544

545

546

547

548

549

550

551

552

553

554

555

556

557

558

559

560

561

562

563

564

565

566

567

568

569

570

571

572

573

574

575

576

577

578

579

580

581

582

583

584

585

586
// creates a feature type so that the new position update can be sent to

// the server

FeatureType subType $=$ new FeatureType () ;

// gets the subtype of the feature being sent

subType $=$ editLayer.getTypes ()$[0]$;

// calls the appleEdits function to send the feature to the server applyEdits(GeometryEngine.project(locx, locy, webMercator), subType, editLayer);

\}

public void applyEdits(Geometry geometry, FeatureType subType, ArcGISFeatureLayer featureLayer) \{

// creates a calendar object with current time

Calendar rightNow $=$ Calendar.get Instance () ;

// creates string of the calendar object

String dateString $=$ DateFormat.getDateTimeInstance () . format ( rightNow.getTime());

// creates a graphic to send to the server

Graphic graphic = featureLayer.createFeatureWithType(subType, geometry);

// gets attributes of the feature

Map<String, object> attr = graphic.getAttributes () ;

// assigns the attributes to the feature

attr.put("ObserverID", mEventUser);

attr.put("ConfidenceRating", $\theta$ );

attr.put ("eventType", $\theta$ );

attr.put("Permissions", 2);

attr.put("Date", rightNow);

// create a new graphic with the attributes. attributes are immutable Graphic newGraphic = new Graphic(geometry, graphic.getSymbol(), attr, graphic.getInfoTemplate());

// applies edits

featureLayer.applyEdits(new Graphic[] \{ newGraphic \}, null, null, new CallbackListener $\langle$ FeatureEditResult [][]$>()\{$

public void onError(Throwable error) \{

Toast.makeText (getApplicationContext(), "error", 5000) . $\operatorname{show}()$;

\}

public void onCallback(FeatureEditResult[][] editResult) \{

if (editResult[0] != null \&\& editResult[0][0] != null 
592

593

594

595

596

597

598

599

600

601

602

603

604

605

606

607

608

609

610

611

612

613

614

615

616

617

618

619

620

621

622

623

624

625

626

627

628

629

630

631

632

633

634

635

636

637

638

639

640

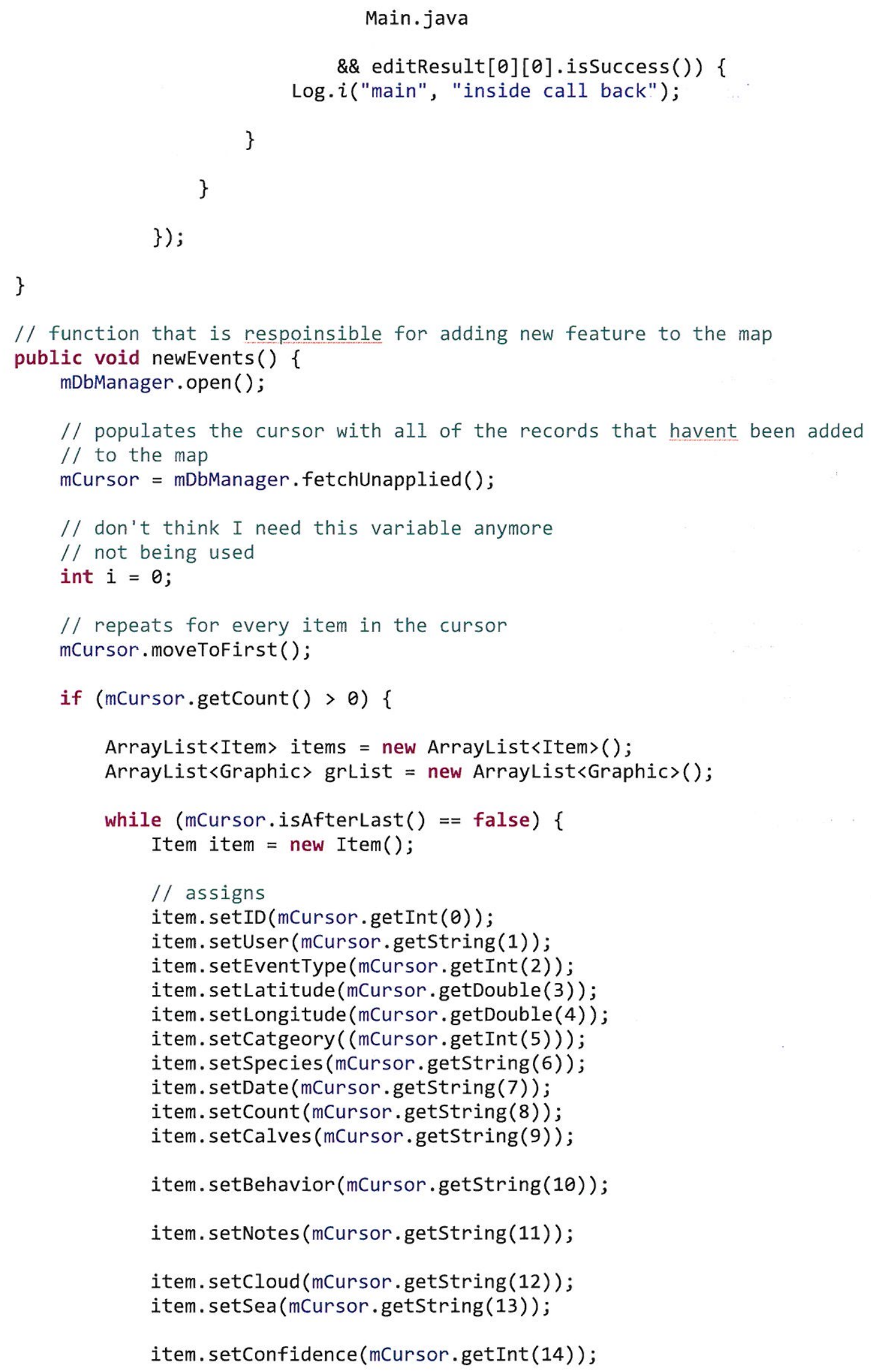

Main.java 
Main.java

641

642

643

644

645

646

647

648

649

650

651

652

653

654

655

656

657

658

659

660

661

662

663

664

665

666

667

668

669

670

671

672

673

674

675

676

677

678

679

680

681

682

683

684

685

686

687

688

689

690

691

692

693

694
item.setPermissions(mCursor.getInt(15));

mDbManager.UpdateApplied(item.getID( ));

items. add (item);

$i=i+1$;

mCursor.moveToNext();

\}

for (Item item : items) \{

// creates a point for each item in the item array

Point pointwm $=$ GeometryEngine $\cdot \operatorname{project}($ item.getLongitude () , item.getLatitude(), webMercator);

HashMap<String, Object $>$ attrs = new HashMap<String, Object $>()$;

attrs.put("SpeciesCategory", item.getCatgeory());

// creates a graphic that can be rendered

Graphic gr = new Graphic(pointwm, null, attrs, null);

Toast.makeText (getApplicationContext(), "" + item.getCatgeory(), Toast.LENGTH_SHORT).show();

// adds graphic to the graphic list

$\operatorname{grList} . \operatorname{add}(\mathrm{gr})$;

\}

Graphic[] grs = new Graphic[grList.size()];

grs $=$ grList.toArray (grs);

Featureset $f s=$ new FeatureSet () ;

// adds graphics to the feature set

fs.setGraphics(grs);

Options options = new Options ( );

options.mode $=$ MODE. SNAPSHOT;

mDbManager.close();

try \{

// adds the feature set to the map

fLayer = new ArcGISFeatureLayer(layerDefinition, fs, options); map.addLayer(fLayer);

\} catch (Exception e) \{

// TODO Auto-generated catch block

e.printStackTrace();

\}

\}

else \{

Toast.makeText(getApplicationContext(), "no new events", 
Main.java

695

696

697

698

699

700

701

702

703

704

705

706

707

708

709

710

711

712

713

714

715

716

717

718

719

720

721

722

723

724

725

726

727

728

729

730

731

732

733

734

735

736

737

738

739

740

741

742

743

744

745

746

747

748

Toast.LENGTH_SHORT).show ( );

\}

// /no longer using onActivity for Result, can delete @override protected void onActivityResult(int requestCode, int resultCode, Intent data) \{ // TODO Auto-generated method stub Log.i("main", "inActivityResult"); super.onActivityResult (requestCode, resultCode, data);

if (data.getExtras().containsKey("syncResult")) \{ Log.i("main", "synced"); Toast toast $=$ Toast. makeText(this, "observation saved", 5000); 
Main.java

749

750

751

752

753

754

755

756

757

758

759

760

761

762

763

764

765

766

767

768

769

770

771

772

773

774

775

776

777

778

779

780

781

782

783

784

785

786

787

788

$789\}$

790

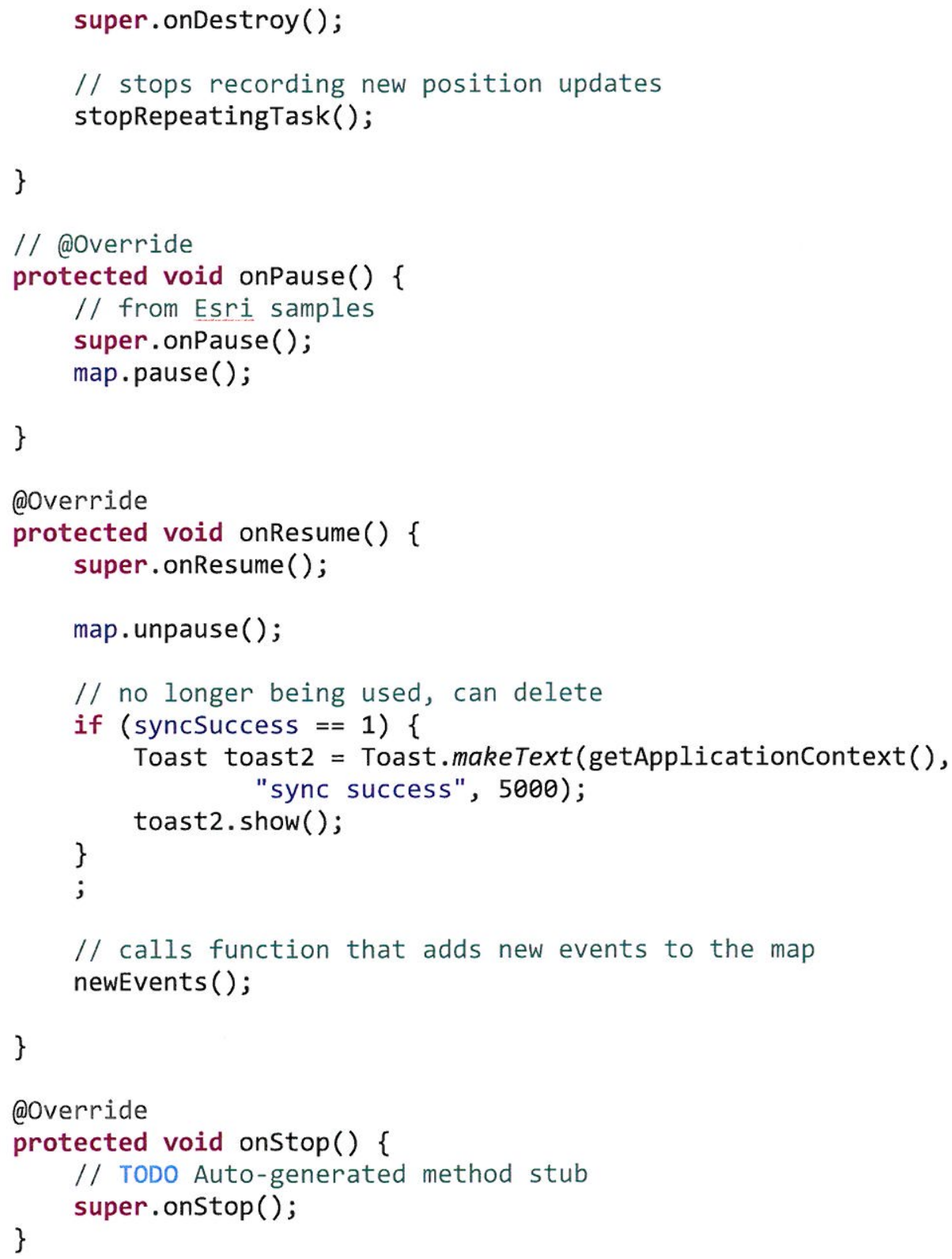

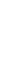



Home. java

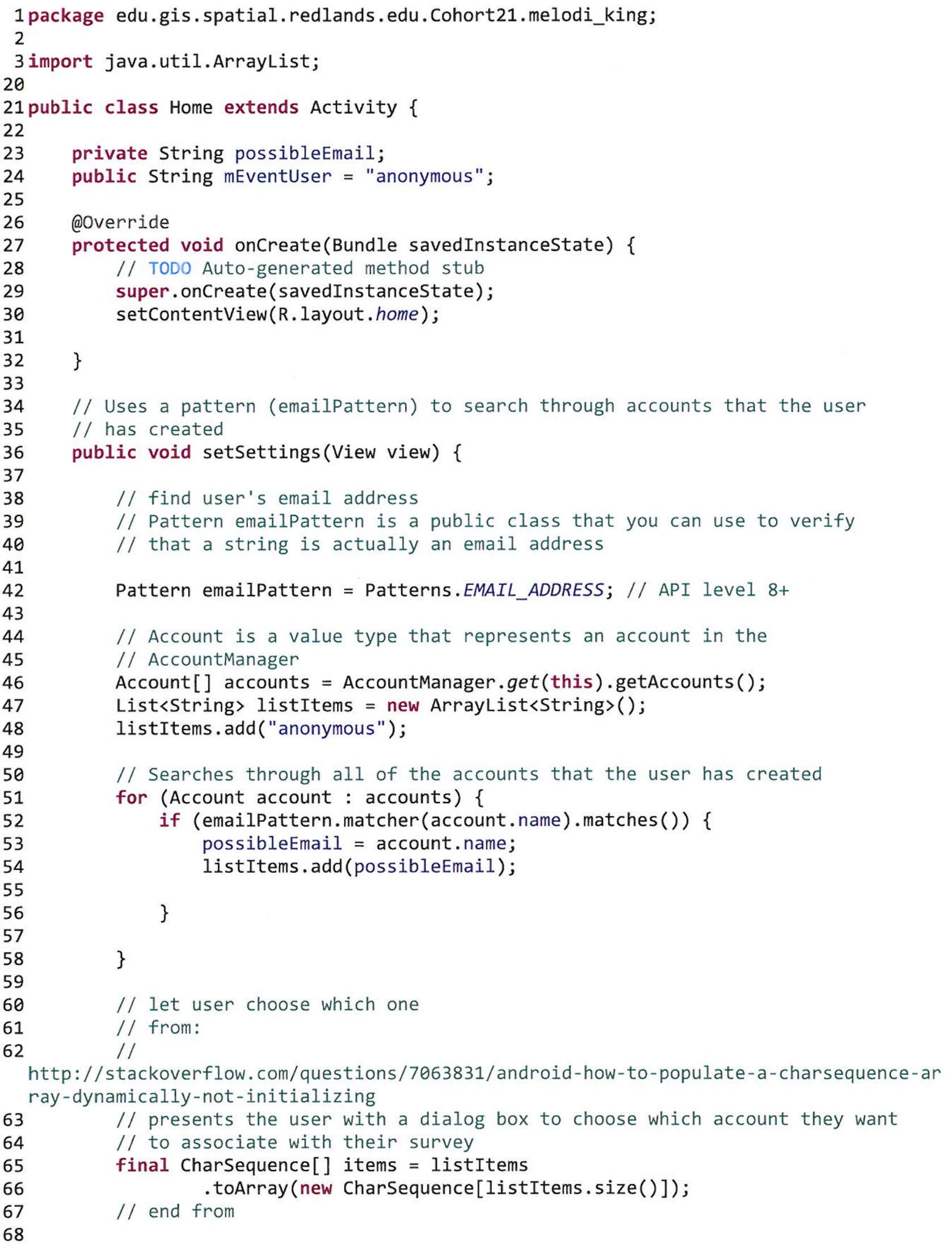


Home.java

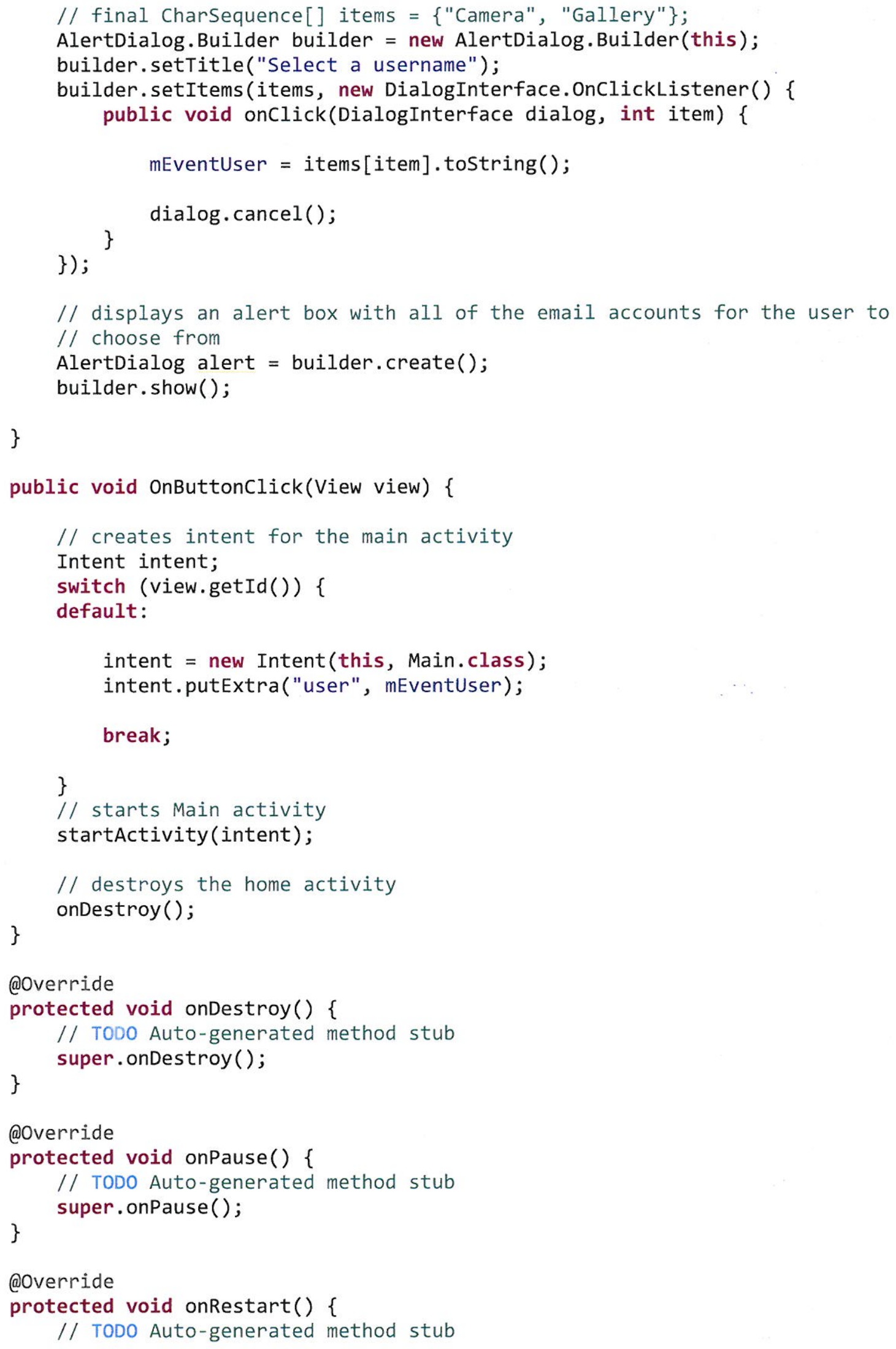


Home.java

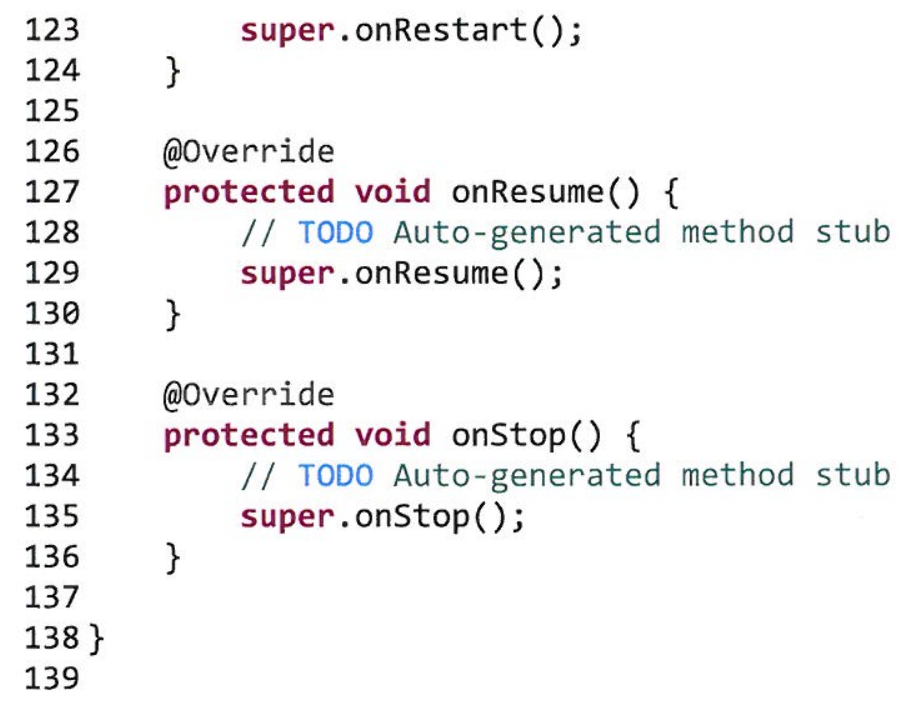





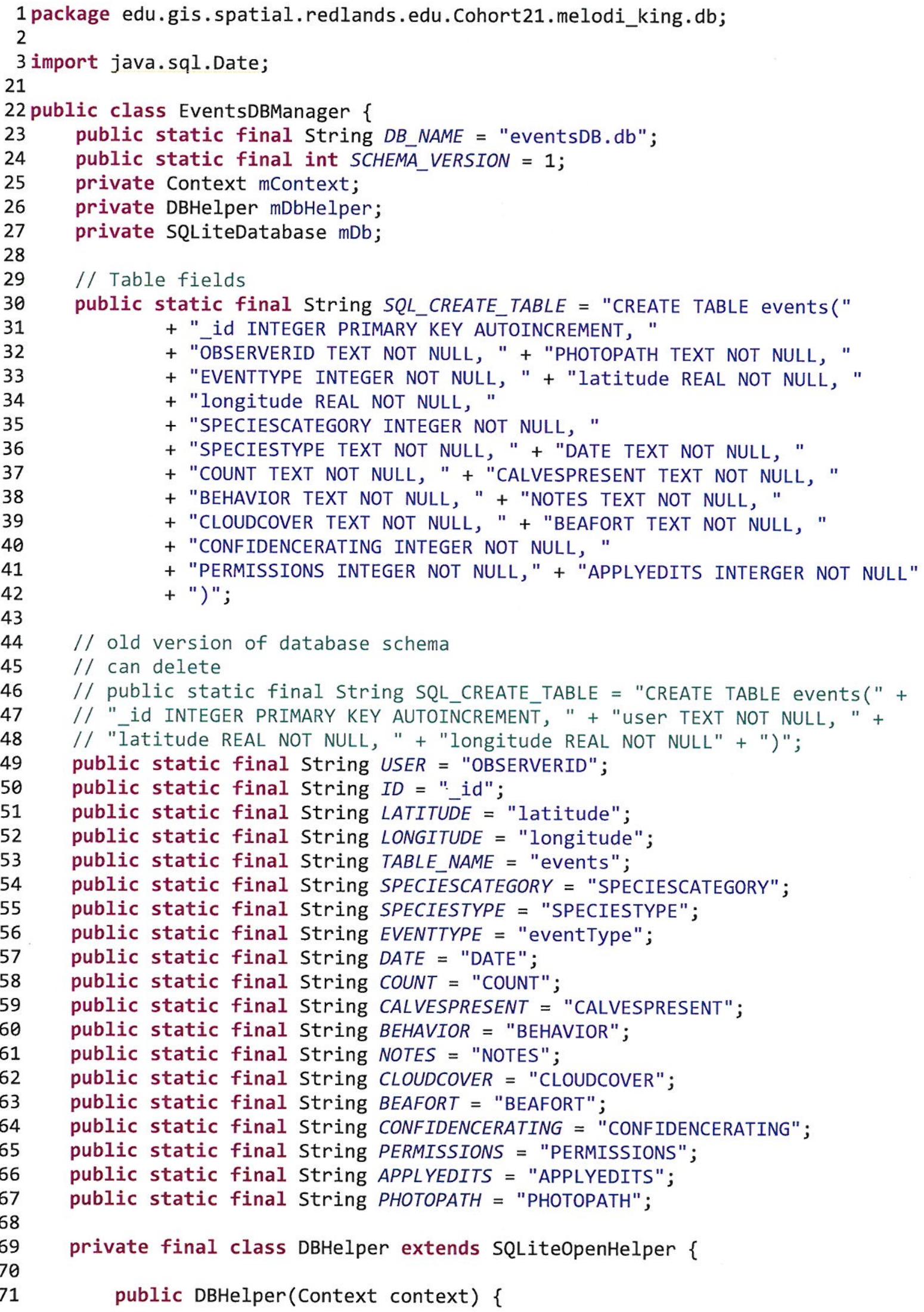


EventsDBManager . java

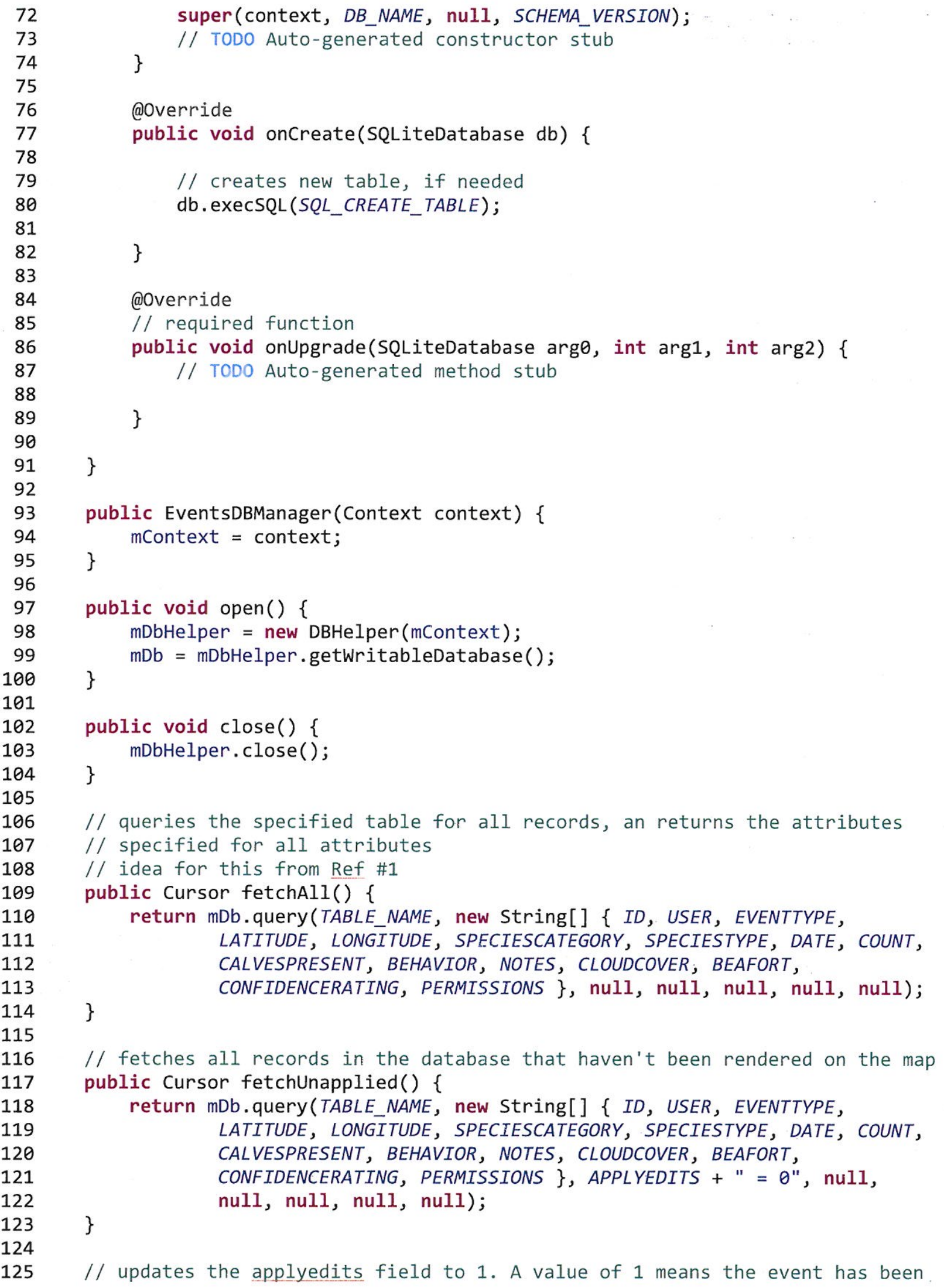




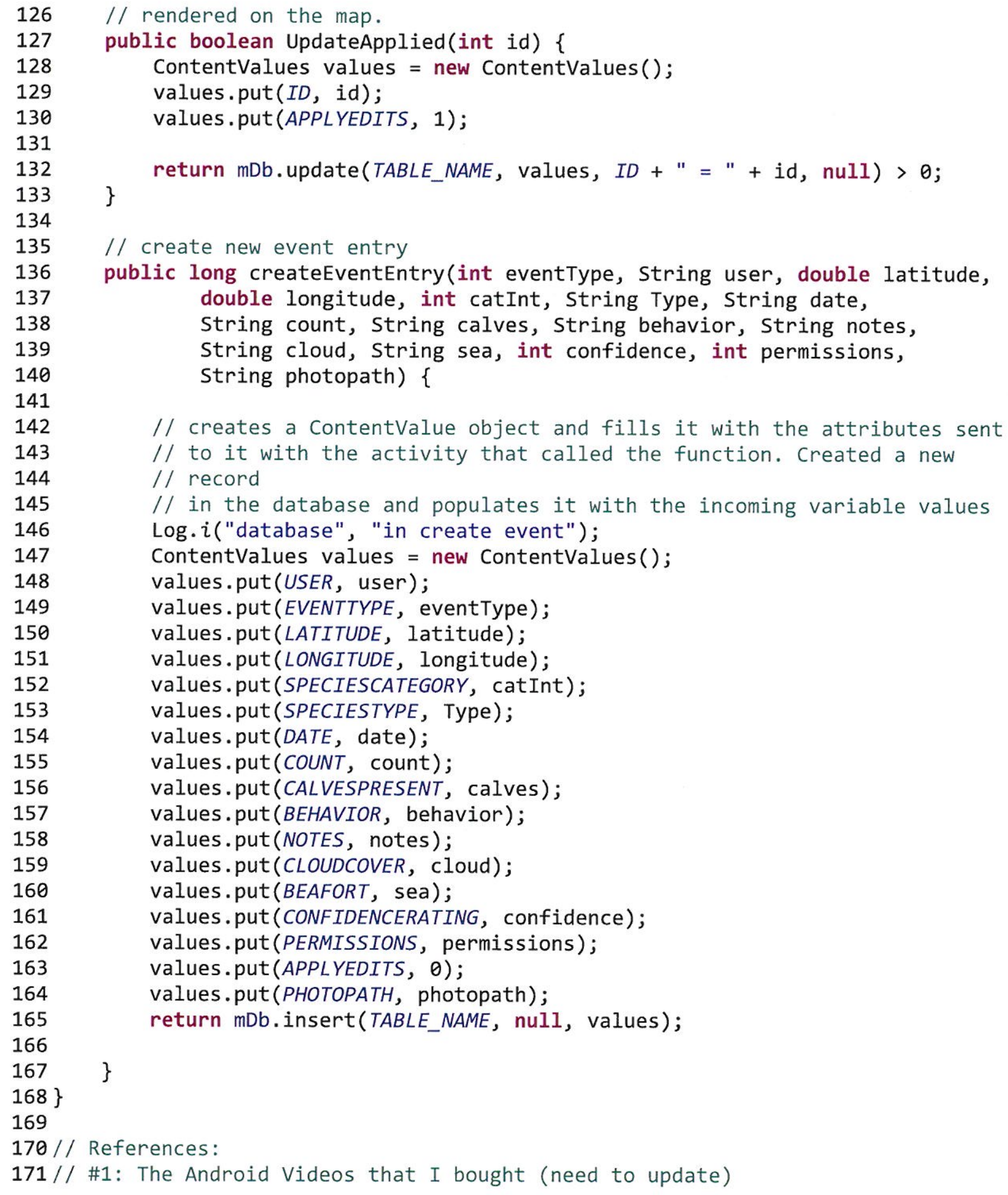



Appendix C. Web Application HTML and Javascript Code 



\section{myobs.html}

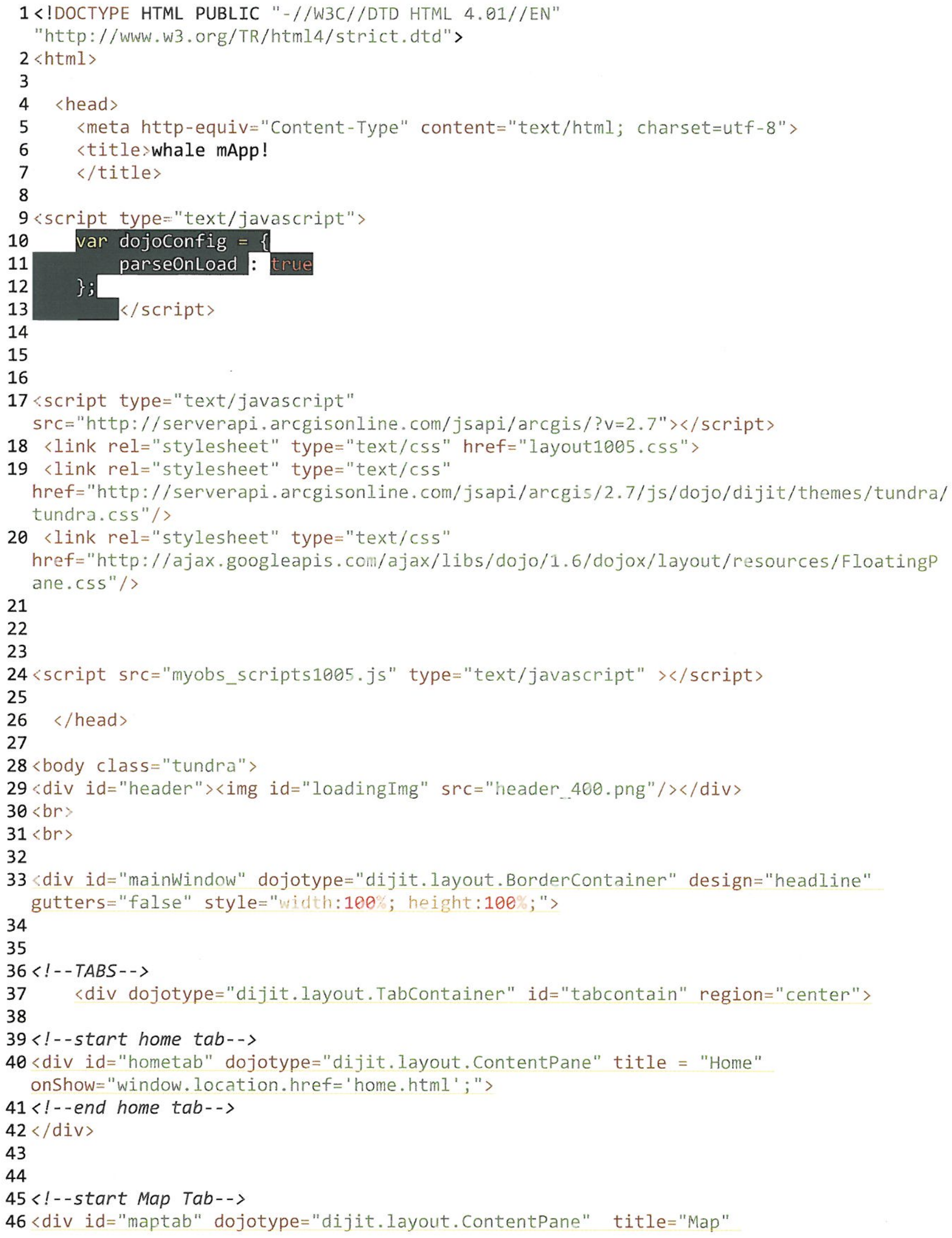


myobs.html

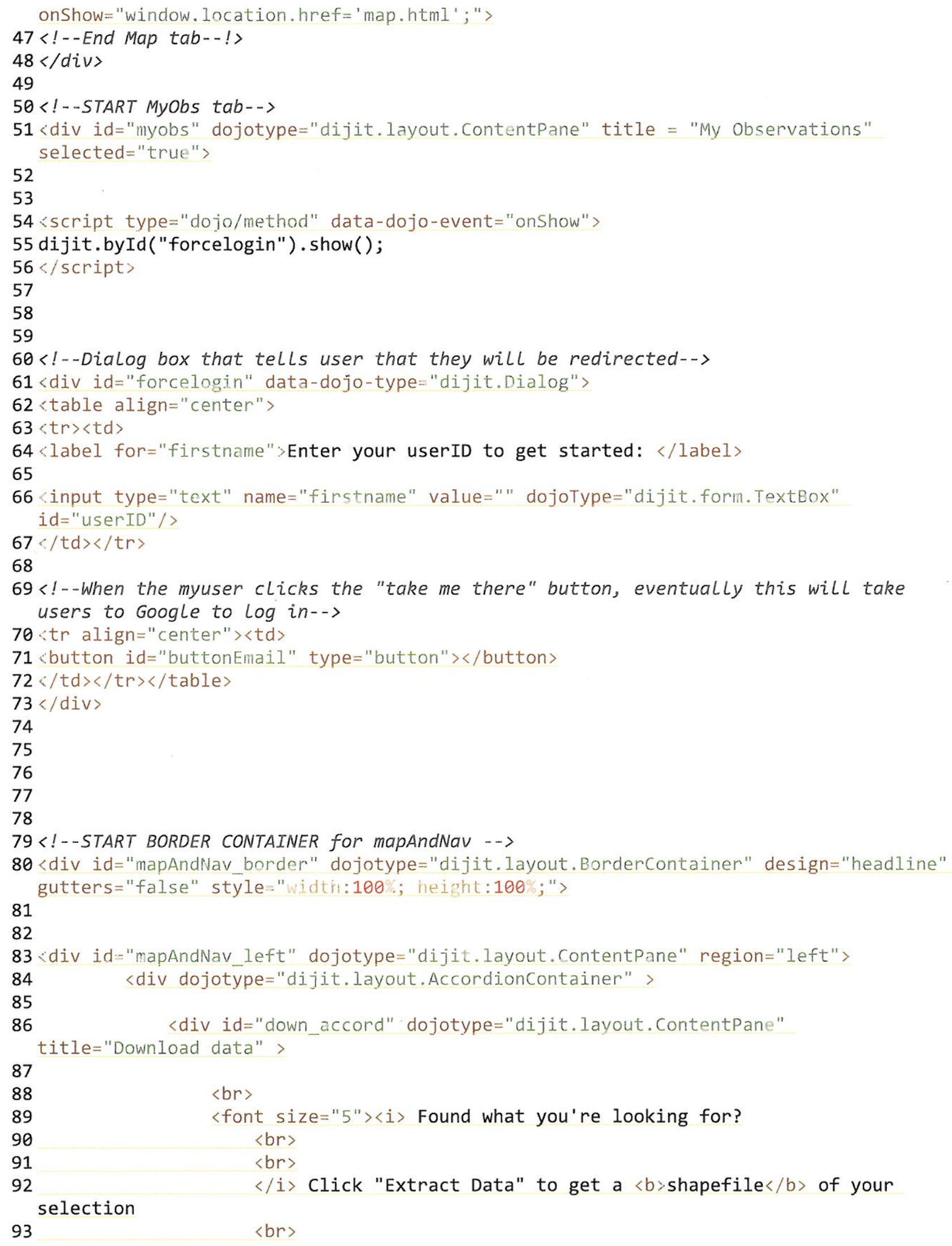




\section{myobs.html}

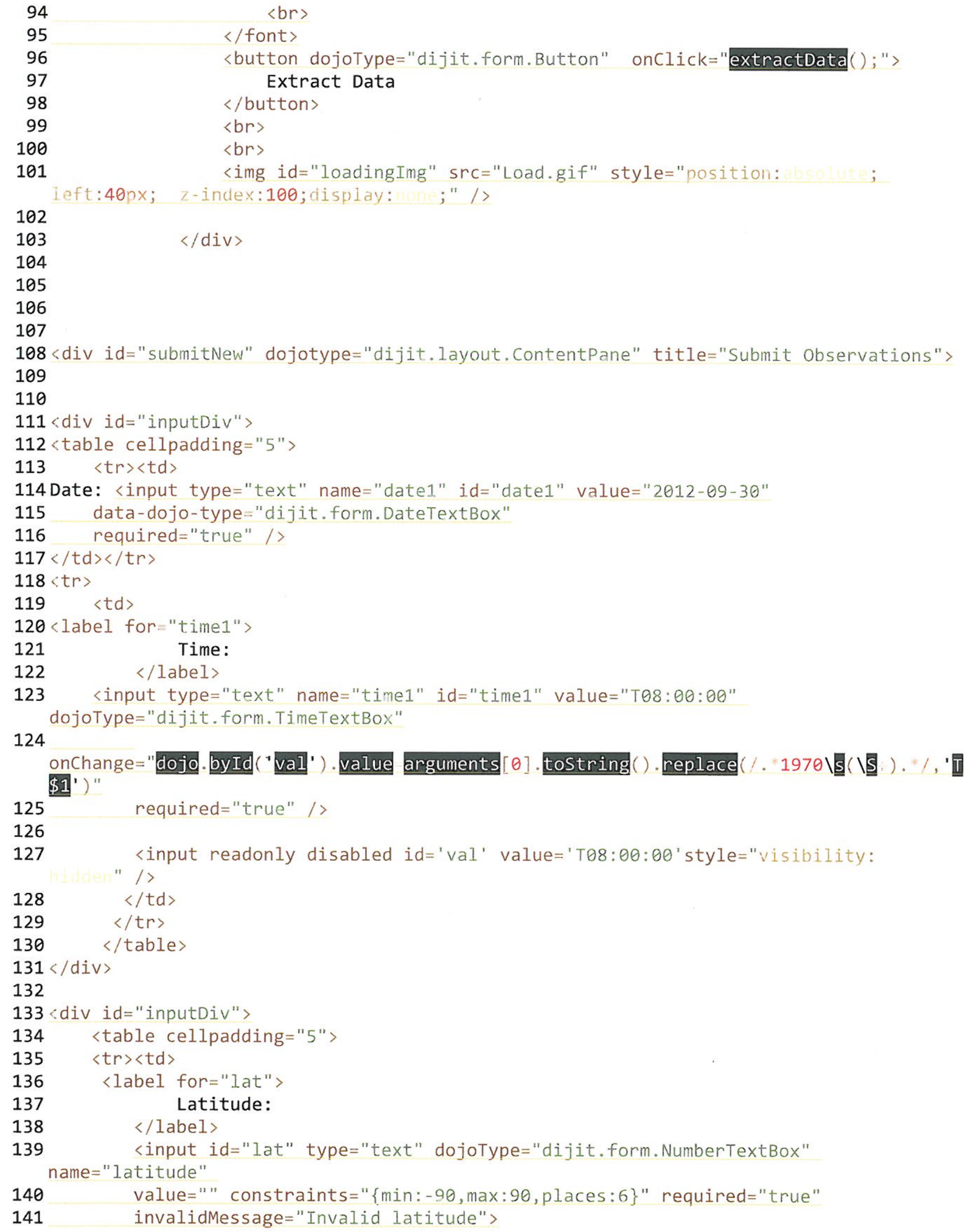


myobs.html

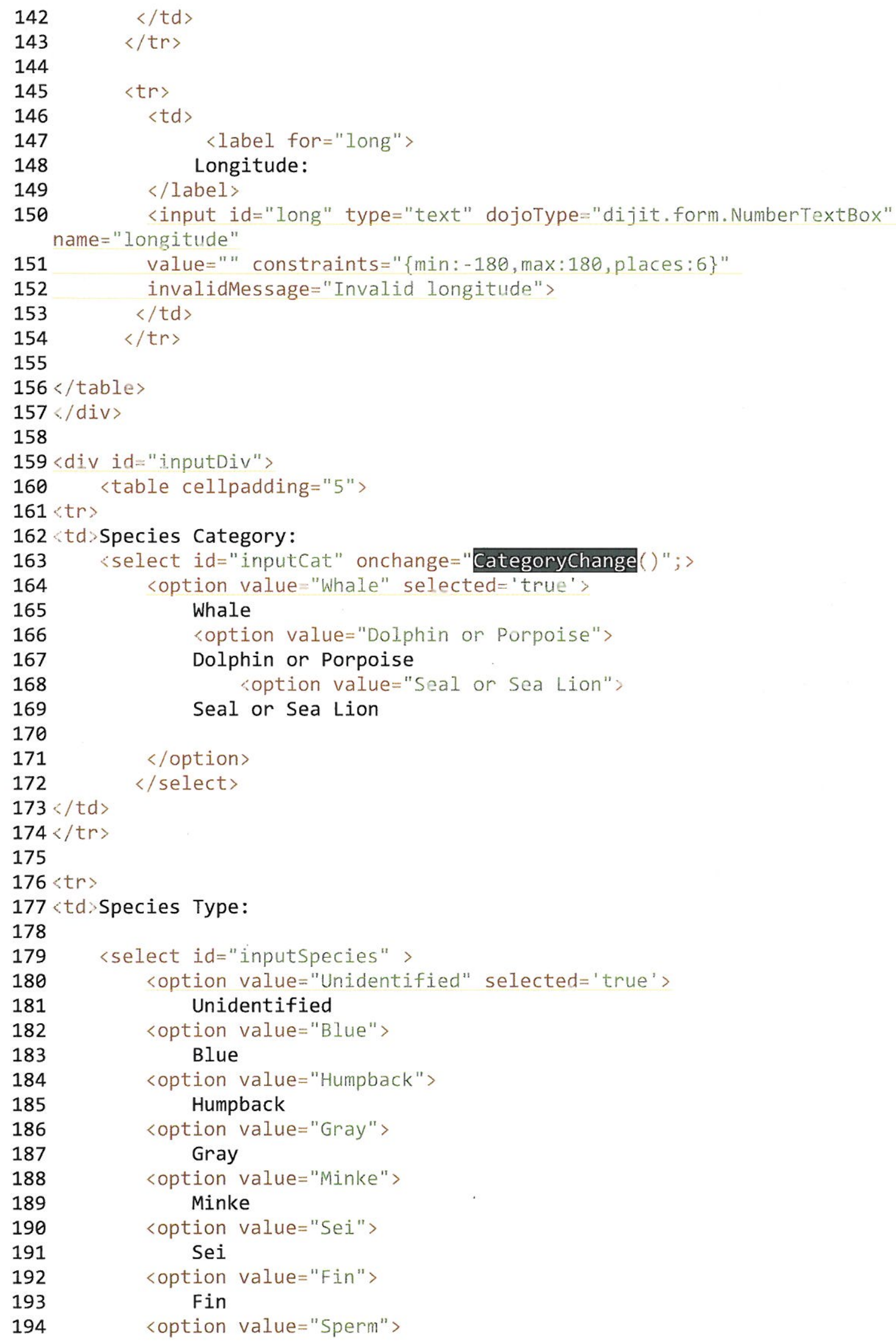


myobs.html

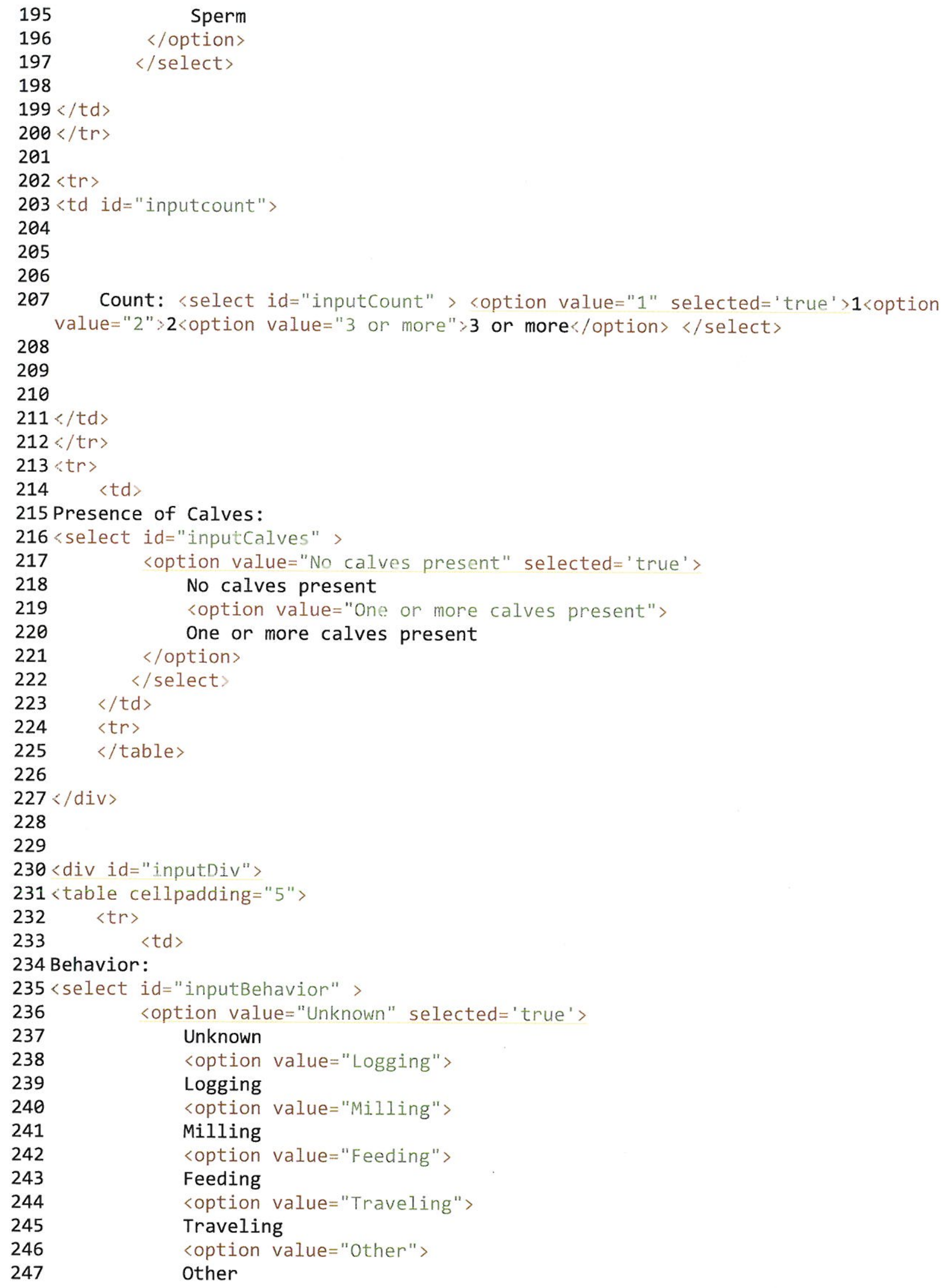


myobs.html

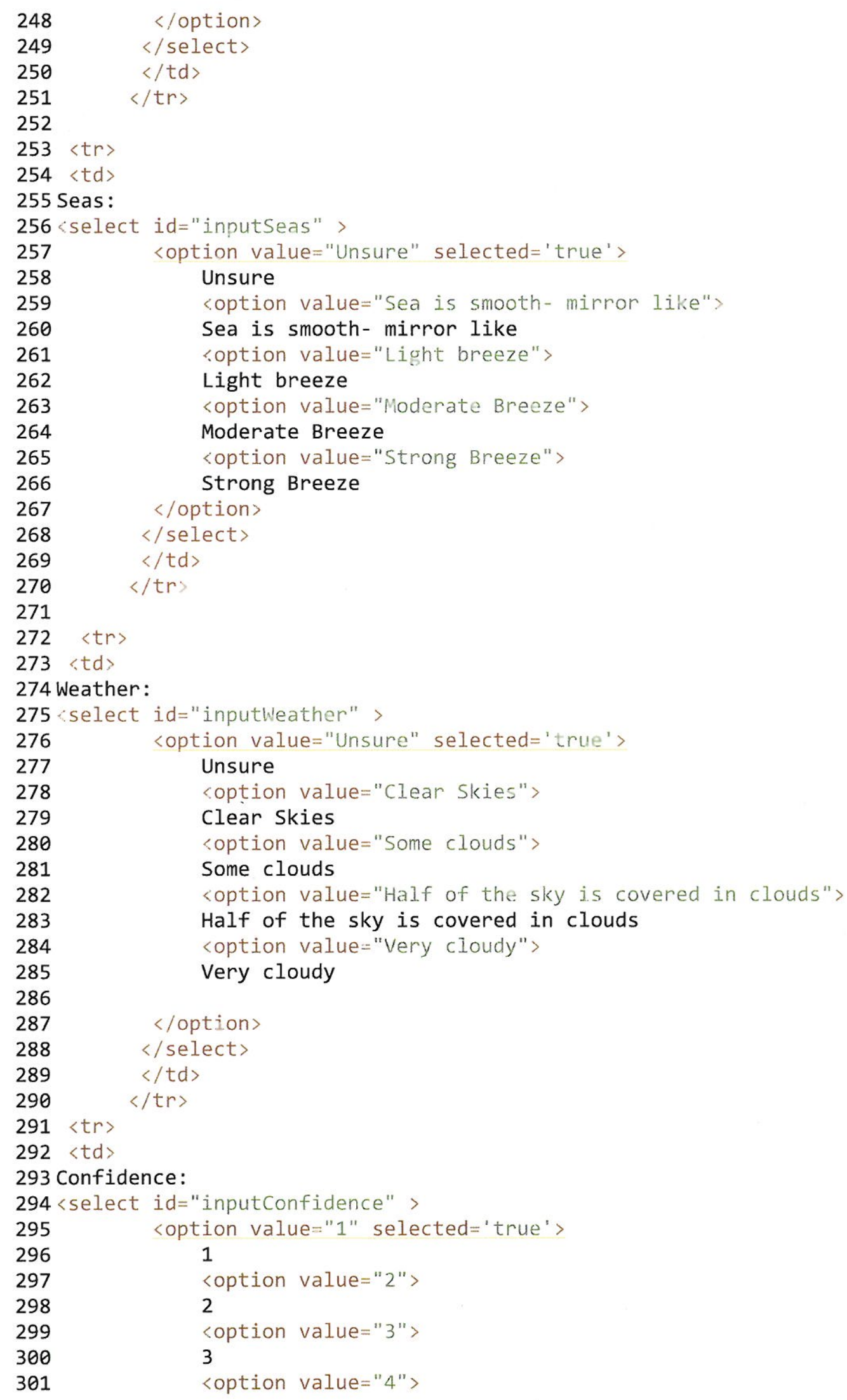


myobs.html

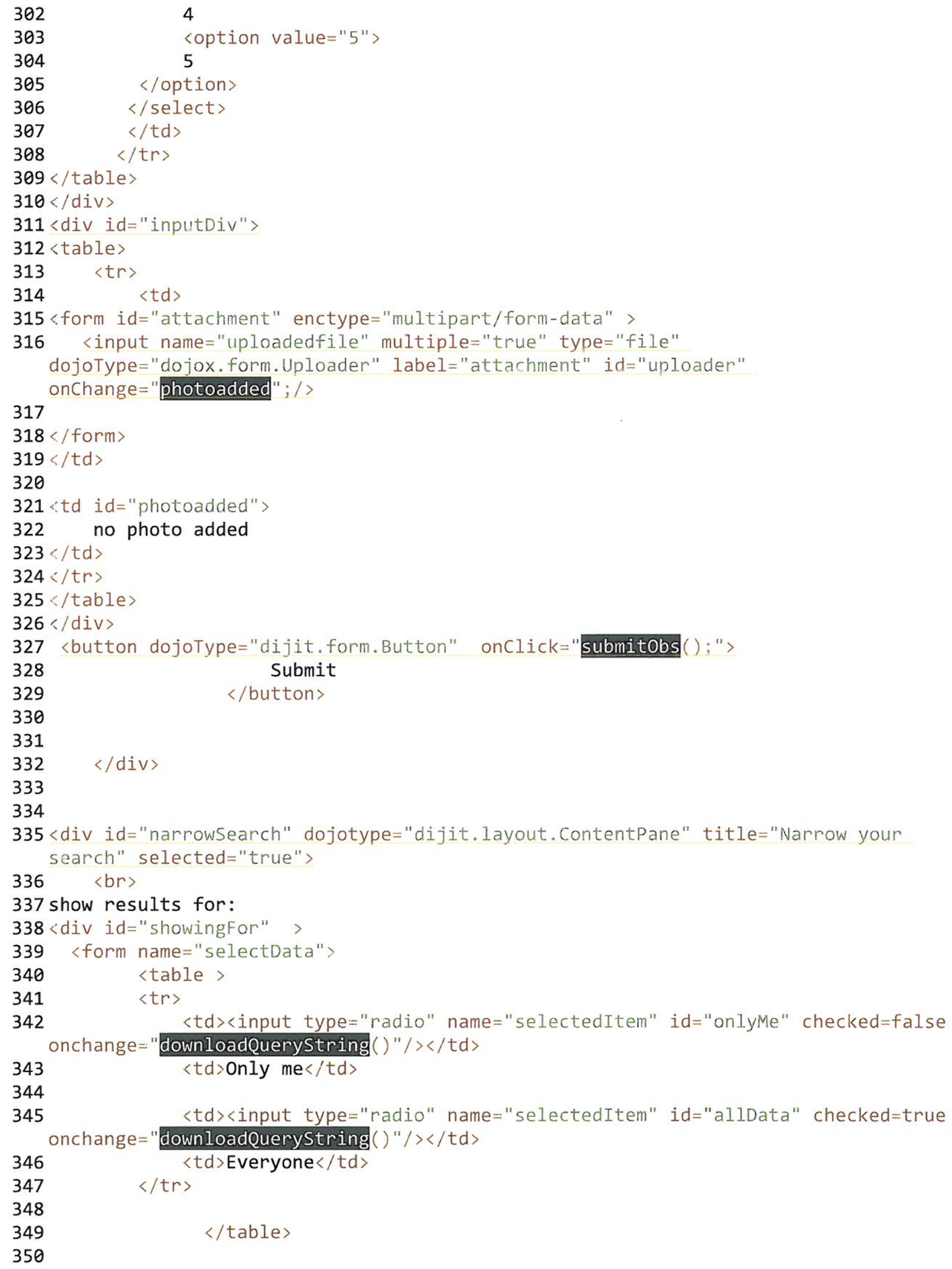


myobs.html

351

352

353

354

355

$356<!--$ end showingFor-->

$357\langle/$ div $\rangle$

358

359

360

361 date range:

362 〈div id="accordianDate" >

363

$364\langle$ table id="dateTable">

365

$366\langle t r\rangle$

$367\langle t d\rangle$

368 from:

$369\langle/$ td $\rangle$

$370<t d>$

$371<!--$ list for users to specify a start month -->

372 select id="monthBeg" onchange="datechange ()" >

373

374

375

376

377

378

379

380

381

382

383

384

385

$386\left\langle\right.$ option value $\left.=" 05^{\prime \prime}\right\rangle$

387

388

389

390

391

392

393

394

395 <option value $=" 08$ " >

396

397

398 <option value $=" 09 "$ "

399

400

coption value $=" 01$ " selected $=$ 'true'> Jan

$<$ /option>

<option value $=" 02$ " >

Feb

〈option value $=" 03 "$ >

Mar

option value =" $\theta 4$ ">

402

403

coption value $=" 06 "\rangle$

Jun

</option>

coption value $=" 07 "\rangle$

Jul

$</ o p t i o n>$

$404\langle$ option value $=" 11 "\rangle$ 
myobs.html

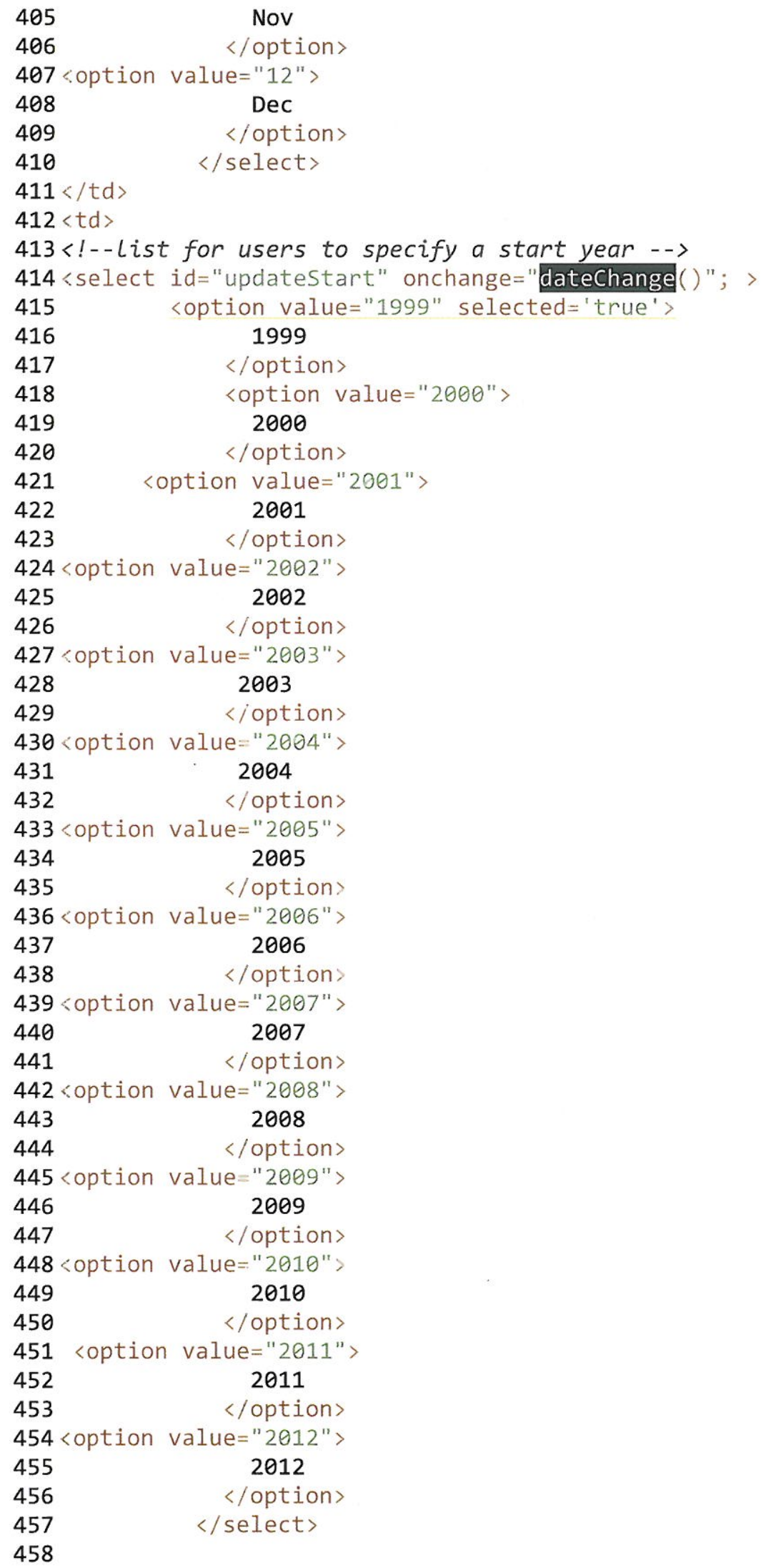


myobs.html

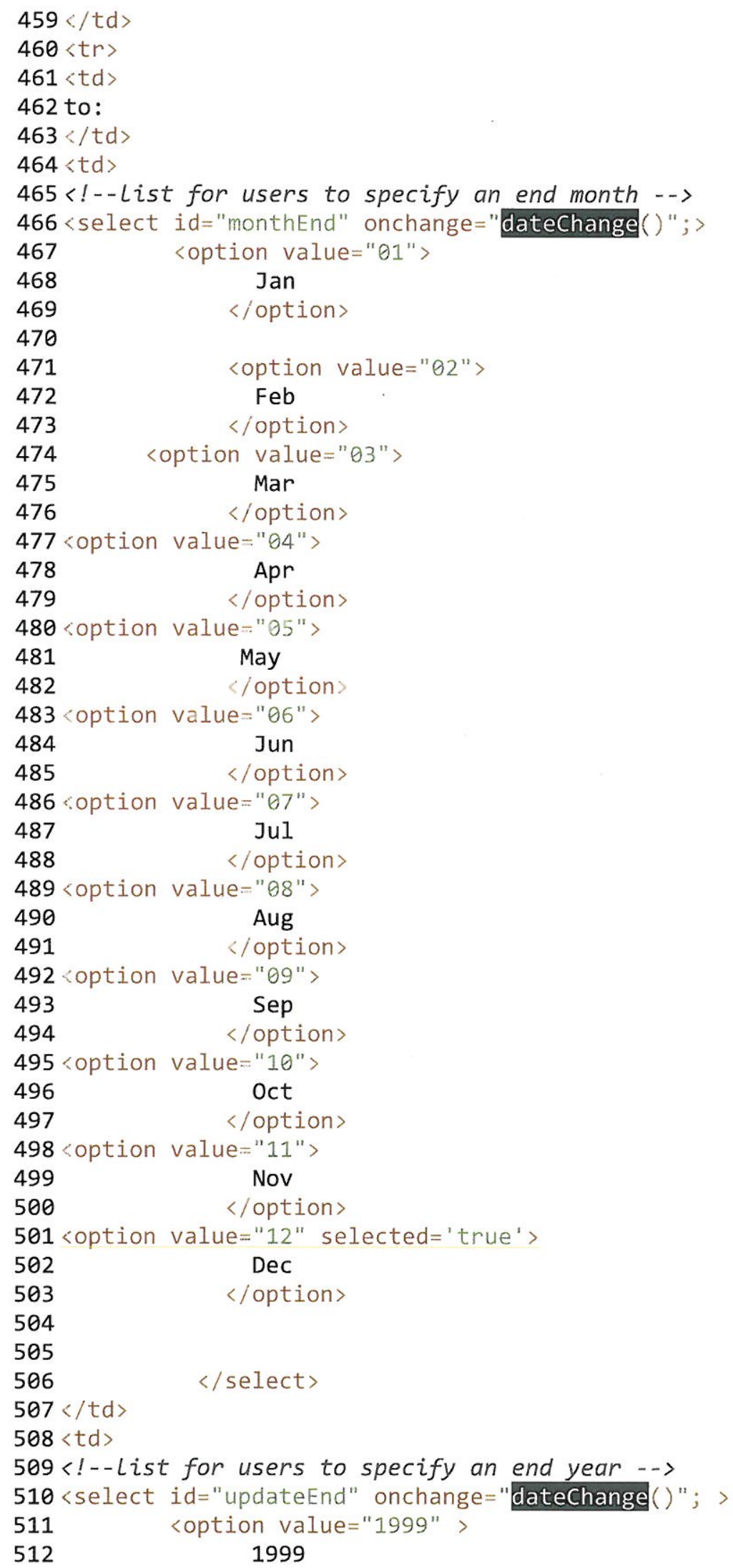


myobs.html

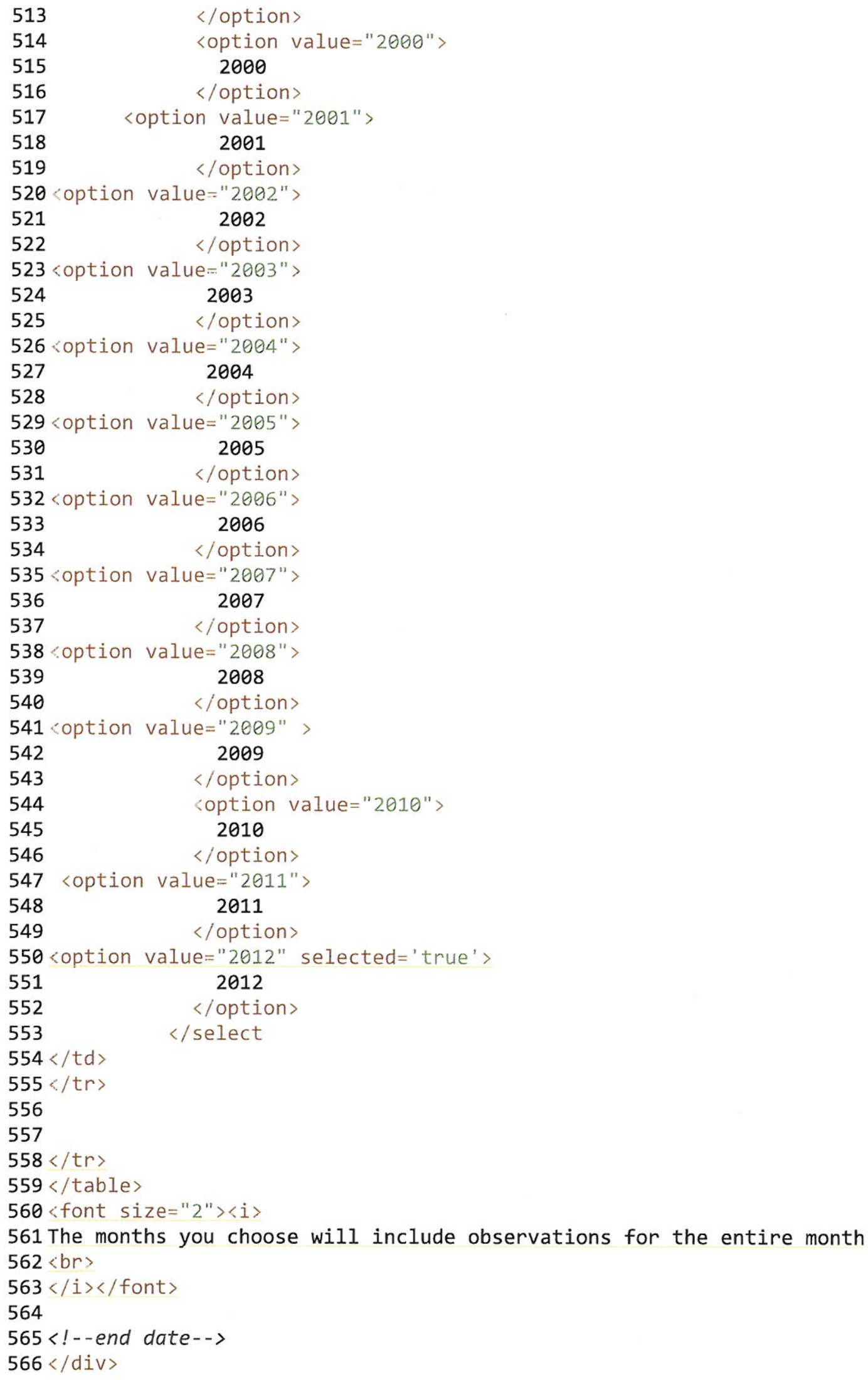


myobs.html

567

568 event type:

$569<$ !--start event accordian container-->

$570\langle$ div id="accordianEvents"〉

$571\langle$ table $>$

$572\langle t r\rangle$

$573\langle t d\rangle$

574 ¿input id="obsBox" ><label for="obsBox" >observations $\langle/$ label $\rangle$

$575</$ td $>$

$576</$ tr $>$

$577\langle t r\rangle$

$578\langle t d\rangle$

$579\langle$ input id="trackBox" ><label for ="trackBox" >tracks /label >

580

$581\langle/ t d\rangle$

$582\langle/$ tr $>$

$583</$ table $>$

584

585

$586<!--$ end event accordian-->

$587\langle/$ div $>$

588

589 species type:

590 〈div id="accordianspecies" >

591

$592<!--T a b l e$ of species options-->

$593\langle$ table $\rangle\langle t r\rangle\langle t d$ id $=$ "species" $\rangle$

594

$595\langle$ input id="whaleBox" > label for="whaleBox" $\rangle$ whales $\langle/$ label $\rangle\langle/$ td $\rangle$

$596\langle/$ tr $>$

$597\langle t r\rangle$

598 〈td id = "species" >

$599\langle$ input id="dolphBox" ><label for "dolphBox"> dolphins and porpoises $\langle/$ label $\rangle\langle/$ td $\rangle$

600

$601\langle$ tr $r$

$602<$ td id = "species" >

$603\langle$ input id="sealBox" $\rangle\langle$ label for $="$ sealBox" $\rangle$ seals and sea lions $\langle/$ label $\rangle\langle/$ td $\rangle$ 604

$605\langle/ t r\rangle$

606

$607\langle/ t r\rangle$

$608\langle$ td id $=$ "species" >

$609\langle$ input id="otherBox" $\rangle\langle$ label for="otherBox" $\rangle$ other species $\langle/$ label $\rangle\langle/$ td $\rangle$

610

$611\langle/ t r\rangle$

$612</$ table $>$

613

$614<!--e n d$ species accordian-->

$615\langle/$ div $\rangle$

616

617

618

619 
myobs.html

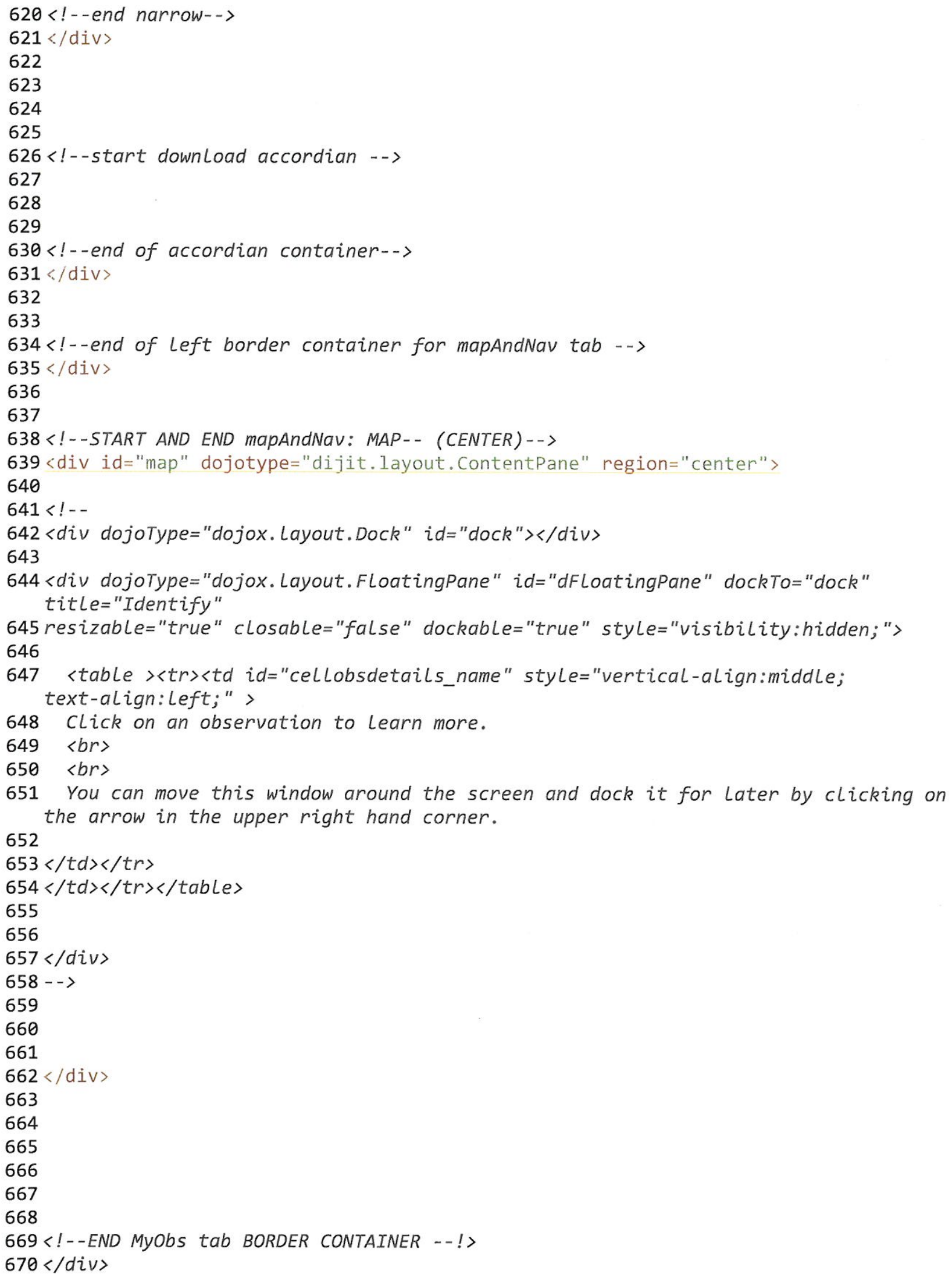


myobs.html

$671\langle!--E N D$ MyObs tab--!>

$672\langle/ d i v\rangle$

673

674

675

676

$677<!--s t a r t$ Learning Tab-->

678 <iv id="learn" dojotype="dijit.layout. ContentPane" title="Learn" onShow="window. location. href=' learn.html';">

$679<!--E N D$ Learning Tab--! >

$680\langle/ d i v\rangle$

681

682

683

$684\langle$ !--END TAB CONTAINER --! $\rangle$

$685\langle/ d i v>$

686

$687\langle$ !--END BORDER CONTAINER--!〉

$688\langle/ d i v\rangle$

689

$690</$ body $>$

$691\langle/ h t m L\rangle$ 
myobs_scripts1005.js

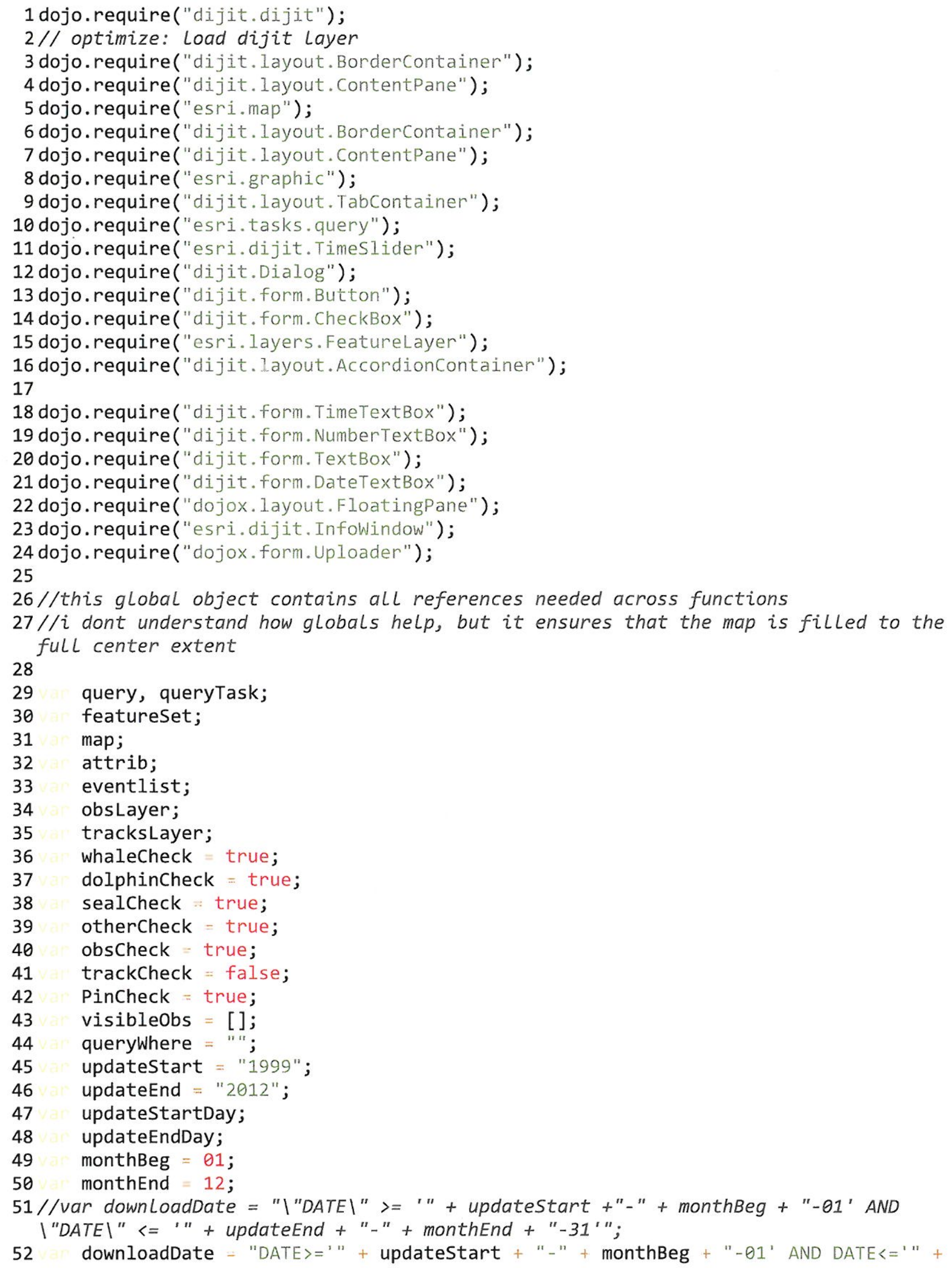


myobs_scripts1005.js

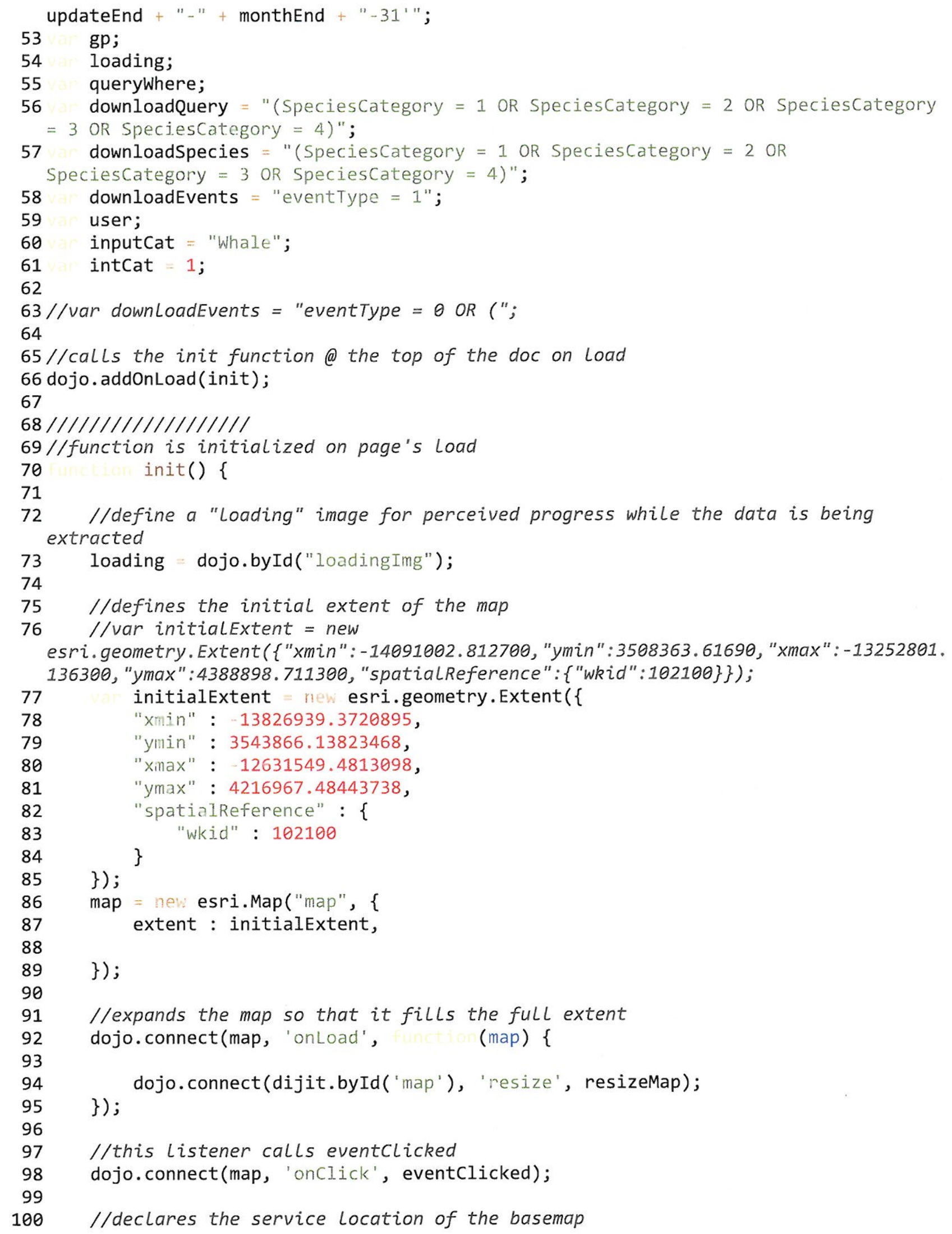


myobs_scripts1005.js

101 basemap = new

esri.layers. ArcGISTiledMapServiceLayer("http://server.arcgisonline.com/ArcGIS/rest/s ervices/Ocean Basemap/MapServer");

102

103

104

105

esri.layers.FeatureLayer("http://gis.spatial.redlands.edu/ArcGIS/rest/services/melod i_king/events/Featureserver/1", \{

106

107

108

109

110

111

112

113

114

115

116

117

118

esri. Layers. Featurelayer i_king/eventsFinal/MapServer//4", \{

119

120

121

122

123

124

125

126

127

128

//only displays events of type 2 (these are observations)

$/ /$ tracksLayer. setDefinitionExpression ("eventType $=2^{\prime \prime}$ );

//adds observations to the Lap

//map.addLayer(tracksLayer);

//declares new gp service

esri.tasks.Geoprocessor("http://gis.spatial.redlands.edu/ArcGIS/rest/services/melodi_ king/events/GPServer/eventExtract");

129

130

131

132

133

134

135

136

137

138

139

140

141

142

143

144

145 //check boxes for types of marine mammals //whale checkbox

whaleBox = new dijit.form. CheckBox ( \{

name : "whaleBox",

value : "agreed",

checked : true,

onchange :

$$
\begin{aligned}
& \text { it }(b-1)\{ \\
& \text { whalecheck = true; }
\end{aligned}
$$

//calls the updateVisiblespecies function updateVisibleSpecies()

\} else \{

whaleCheck = false; 


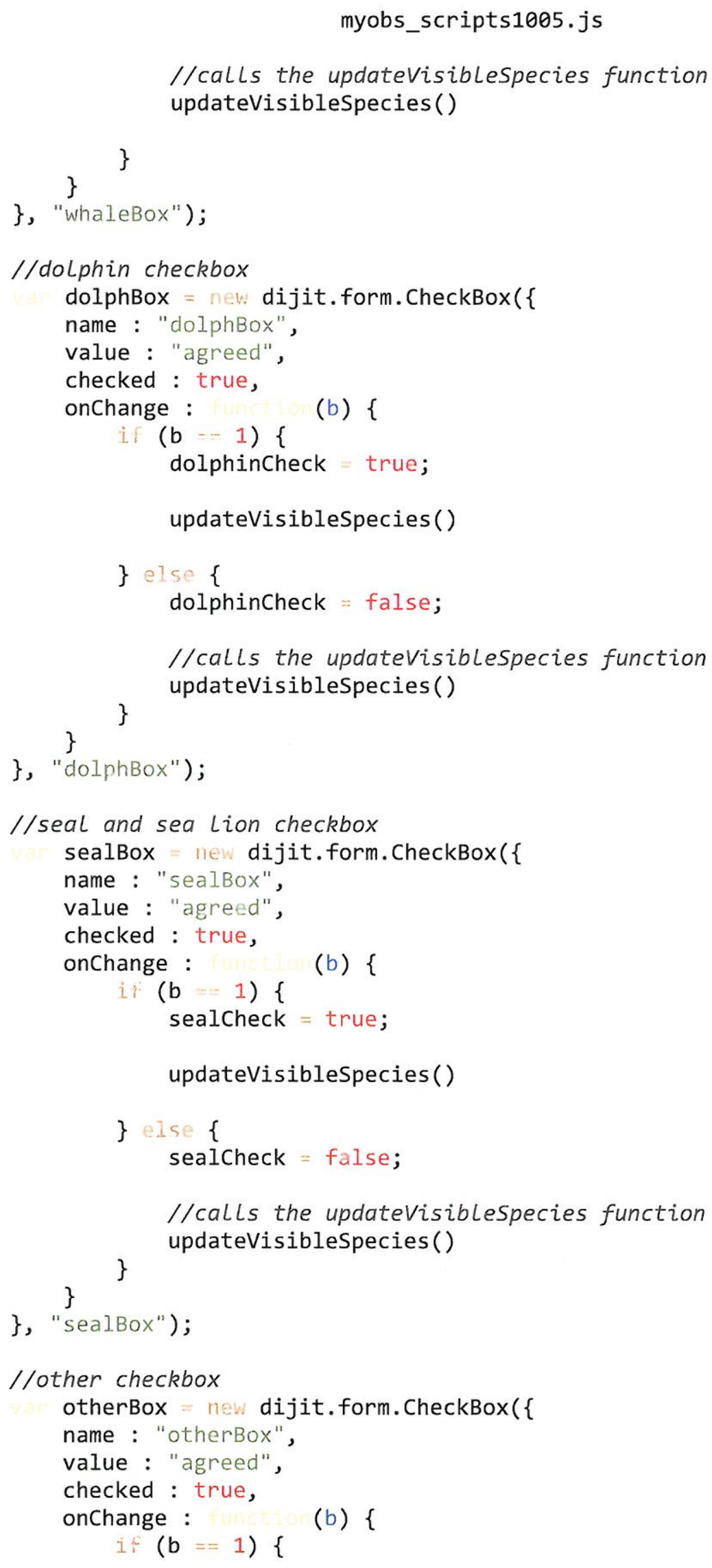

